University of Nebraska - Lincoln

DigitalCommons@University of Nebraska - Lincoln

$10-5-2020$

Steganoderma Stafford, 1904 (Digenea: Zoogonidae:

Lepidophyllinae) from Two Species of Rockfishes from Deep

Waters off Oregon Including a New Species and an Updated Key

to Species of This Genus

Charles K. Blend

Corpus Christi Museum of Science and History, ilovethesea@att.net

Gábor R. Rácz

University of Nebraska - Lincoln, gracz2@unl.edu

Follow this and additional works at: https://digitalcommons.unl.edu/manter

Part of the Biodiversity Commons, Parasitology Commons, and the Zoology Commons

Blend, Charles K. and Rácz, Gábor R., "Steganoderma Stafford, 1904 (Digenea: Zoogonidae:

Lepidophyllinae) from Two Species of Rockfishes from Deep Waters off Oregon Including a New Species and an Updated Key to Species of This Genus" (2020). MANTER: Journal of Parasite Biodiversity. 14. https://digitalcommons.unl.edu/manter/14

This Article is brought to you for free and open access by the Parasitology, Harold W. Manter Laboratory of at DigitalCommons@University of Nebraska - Lincoln. It has been accepted for inclusion in MANTER: Journal of Parasite Biodiversity by an authorized administrator of DigitalCommons@University of Nebraska - Lincoln. 


\title{
Steganoderma Stafford, 1904 (Digenea: Zoogonidae: Lepidophyllinae) from Two Species of Rockfishes from Deep Waters off Oregon Including a New Species and an Updated Key to Species of This Genus
}

\author{
Charles K. Blend ${ }^{1 *}$ and Gabor R. Racz ${ }^{2}$ \\ 1 Corpus Christi Museum of Science and History, 1900 N. Chaparral Street, Corpus Christi, Texas 78401, \\ USA (ilovethesea@att.net) \\ 2 Harold W. Manter Laboratory of Parasitology, W529 Nebraska Hall, University of Nebraska-Lincoln, \\ Lincoln, Nebraska 68588-0514, USA (gracz2@unl.edu) \\ * Corresponding author
}

\begin{abstract}
Steganoderma eamiqtrema n. sp. and a single unidentified specimen of Steganoderma Stafford, 1904 (Zoogonidae: Lepidophyllinae) obtained from the intestine of the greenstriped rockfish, Sebastes elongatus Ayres, 1859, and the flag rockfish, Sebastes rubrivinctus (Jordan and Gilbert, 1880) (Scorpaeniformes: Sebastidae), collected from 190-200 m depths off Oregon, USA, are described. The new species is distinguished from its seven other congeners by a diagnostic combination of morphological features including an elongate oval to spindle-shaped body, a clavate to comma-shaped cirrus pouch located in the forebody and hindbody, a bipartite seminal vesicle, a bifurcal or just post-bifurcal genital pore, a larger ventral than oral sucker, and a smooth testes and ovary with a relatively small distance between them. We present an updated key to the eight species now in Steganoderma and provide a list of parasites known from Se. elongatus and Se. rubrivinctus. The discovery of S. eamiqtrema in Se. elongatus represents the second species of zoogonid known from this host, and the finding of Steganoderma sp. in Se. rubrivinctus represents the first report of a digenean from this host species. A detailed discussion also is given of the type species, S. formosum Stafford, 1904, and questions are raised as to whether this species has a worldwide distribution and infects such a wide variety of fish hosts. We present evidence including variation we observed in redescriptions of the type species, query the implausible idea that there could be gene flow between conspecific helminths geographically separated in the North Atlantic and North Pacific Oceans over such a vast geological period, and offer the possibility that some prior reports of S. formosum may, indeed, be S. eamigtrema; all of which suggests $S$. formosum sensu lato may be part of a species complex and not the same worldwide species. Steganoderma is represented in the deep sea by S. eamiqtrema, S. formosum, and Steganoderma sp., and limited speculation is given as to the host specificity of this genus and life history strategies of the new species in deeper waters. Finally, molecular studies of species of Steganoderma are sorely needed (i.e., there is no DNA sequence data currently available in GenBank for any species of this genus), and we suspect that with further molecular, morphological, and life history work, this genus will be taxonomically divided up.
\end{abstract}


Keywords: deep sea, Digenea, flag rockfish, greenstriped rockfish, key, Lepidophyllinae, northeastern Pacific Ocean, Oregon, Scorpaeniformes, Sebastes elongatus, Sebastes rubrivinctus, Sebastidae, Steganoderma eamiqtrema n. sp., Steganoderma sp., Zoogonidae

\section{Introduction}

Steganoderma Stafford, 1904 is a genus within the digenean family Zoogonidae Odhner, 1902, the species of which parasitizes the intestines of marine teleosts worldwide and freshwater teleosts in South America (Bray, 2008b). Currently, Steganoderma is in the zoogonid subfamily Lepidophyllinae Stossich, 1903 and contains seven species (WoRMS, 2020b; Blend et al., 2020); three of these are found in marine waters while the remaining four species inhabit freshwater. Steganoderma was erected by Stafford (1904) with the description of Steganoderma formosum Stafford, 1904 from the ceca and intestine of the Atlantic halibut, Hippoglossus hippoglossus (Linnaeus, 1758) (Pleuronectiformes: Pleuronectidae), collected from the NW Atlantic Ocean off the east coast of Canada. Steganoderma atherinae (Price, 1934) Manter, 1947 (Syn. Lecithostaphylus atherinae Price, 1934) was described by Price (1934) infecting the intestine of the reef silverside, $\mathrm{Hy}$ poatherina harringtonensis (Goode, 1877) (Syns. Atherina arcea Jordan and Gilbert, 1884; Atherina araea Jordan and Gilbert, 1884; Atherina area Jordan and Gilbert, 1884) (Atheriniformes: Atherinidae), found in the NW Atlantic Ocean within Samaná Bay, near Santa Barbara de Samaná, Dominican Republic. The third known marine species of this genus is Steganoderma rhiphidium Wang, 1986 described from the intestine of the bastard halibut, Paralichthys olivaceus (Temminck and Schlegel, 1846) (Pleuronectiformes: Paralichthyidae), from the NW Pacific Ocean off Pingtan County, Fujian Province, China (Wang, 1986). The earliest-described freshwater species of Steganoderma currently recognized is Steganoderma macrophallus Szidat and Nani, 1951 from the intestine of the neotropical silversides Basilichthys microlepidotus (Jenyns, 1841) and Odontesthes smitti (Lahille, 1929) (Syn. Bachmannia smitti [Lahille, 1929]) (Atheriniformes: Atherinopsidae), collected from the Limay and Quequén Grande Rivers, Argentina (Szidat and Nani, 1951). Steganoderma oviformis Szidat, 1962 was described by Szidat (1962) from the intestine of the galaxiid Aplochiton zebra Jenyns, 1842 (Syn. Haplochiton zebra [Jenyns, 1842]) (Osmeriformes: Galaxiidae) from Patagonia, Argentina (Note: Fernandez et al. [2012, p. 234] reported $S$. oviformis from A. zebra collected along the Pacific coast of Chile [i.e., marine waters] stating specifically, "Although Szidat (1962) recorded this species in Patagonian freshwater environments, the slides of deposited specimens indicates Valdivia (Chile) as locality"; Froese and Pauly [2019] suggest that $A$. zebra is amphidromous). Viozzi et al. (2000) described Steganoderma szidati Viozzi, Flores and Ostrowski de Núñez, 2000 from the posterior intestine of the inanga, Galaxias maculatus (Jenyns, 1842), and the galaxiid Galaxias platei Steindachner, 1898 (Osmeriformes: Galaxiidae) from Lake Gutiérrez and other glacial lakes of Andean Patagonia, Argentina. The fourth known freshwater species of this genus is Steganoderma valchetensis Etchegoin, Cremonte and Escalante, 2002, found in the intestine of the naked characin, Gymnocharacinus bergi Steindachner, 1903 (Characiformes: Characidae), collected from Valcheta Creek on the Somuncurá Plateau, northern Patagonia, Argentina (Etchegoin et al., 2002).

The purpose of this study was to add to our knowledge of the Zoogonidae with the description of a new species of Steganoderma discovered in the intestine of the greenstriped rockfish, Sebastes elongatus Ayres, 1859 (Scorpaeniformes: Sebastidae), collected from deep waters off Oregon more than 55 years ago. A single unidentified and damaged specimen of Steganoderma also from the deep waters off Oregon is described from the intestine of the flag rockfish, Sebastes rubrivinctus (Jordan and Gilbert, 1880) (Scorpaeniformes: Sebastidae). We present an updated key to the species of this genus, a listing of the parasites known from Se. elongatus and Se. rubrivinctus (Table 1), and new host and parasite records, and we discuss the presence of Steganoderma within the deep sea.

\section{Materials and Methods}

An unknown number of greenstriped rockfish, Se. elongatus, as well as flag rockfish, Se. rubrivinctus, were collected aboard ship and examined for parasites by the late Dr. James E. McCauley, Department of Oceanography, Oregon State University, Corvallis, Oregon. Fish were collected by otter trawl from $190 \mathrm{~m}$ and $200 \mathrm{~m}$ depths, respectively, within the northeastern Pacific Ocean approximately 40 $\mathrm{km}$ and $13 \mathrm{~km}$ west of Newport, Lincoln County, Oregon. Specimens of Se. elongatus and Se. rubrivinctus were immediately fixed at sea by injecting the body cavity, mouth, and anus with AFA or 4\%-10\% seawater formaldehyde and preserved in $10 \%$ neutral seawater-formalin before being transferred back to the laboratory for autopsy (see Eagle 
Table 1. Parasites reported from the greenstriped rockfish, Sebastes elongatus ${ }^{1}$ Ayres, 1859, and from the flag rockfish, Sebastes rubrivinctus ${ }^{1}$ (Jordan and Gilbert, 1880) (Scorpaeniformes: Sebastidae), including infection site, locality, and references

Locality

References

Sebastes elongatus (greenstriped rockfish)

A-Corynosoma sp. juvenile

B-"Chlamydia-like organism"

C-Nybelinia surmenicola

Okada in Dollfus, 1929 larva

C—Phyllobothrium sp. plerocercoid

CO-Acantholochus venustus

(Kabata, 1971) (Syn. Holobomolochus

venustus Kabata, 1971)

CO_Clavella parva Wilson, 1912

CO_Procolobomatus kyphosus (Sekerak, 1970) (Syn. Colobomatus kyphosus Sekerak, 1970)

D—Deretrema cholaeum

McFarlane, 1936

D—Derogenes varicus (Müller, 1784) Stomach Looss, 1901

D-Fellodistomum sebastodis

Yamaguti and Matumura, 1942

D-Hemiuridae gen. sp. (undetermined)
GI tract

Intestine, mesenteries

Gills

Mesenteries, stomach wall

Nasal cavities

Fins

Cephalic sensory canals

Gall bladder

Gall bladder

Intestine, stomach
Northern Pacific Ocean

Off coast of British

Columbia, Canada

Northeastern Pacific

Ocean

Northern Pacific Ocean

Northeastern Pacific

Ocean

Northeastern Pacific

Ocean

Northeastern Pacific

Ocean

Off British Columbian coast, northern Pacific Ocean

Off British Columbian coast, northern Pacific Ocean

Off British Columbian coast, northeastern Pacific Ocean

Pacific Ocean off Canada
Arai, 19693; Sekerak, 1975; Love and Moser, 1976, ${ }^{3}$ 1983';

Sekerak and Arai, 1977;

Margolis and Arthur, 19793.

Love et al. $2002^{3}$

Kent et al. 1998

Sekerak, 1975;

Love and Moser, 1976,3 19833;

Sekerak and Arai, 1977;

Margolis and Arthur, 19793

Sekerak and Arai, 1977;

Margolis and Arthur, 19793;

Love and Moser, 1983'; Love et al. $2002^{3}$

Sekerak, 1975;

Love and Moser, 1976, ${ }^{3}$ 1983; ;

Sekerak and Arai, 1977;

Margolis and Arthur, $1979^{3}$

Sekerak, 1975:

Love and Moser, 1976, ${ }^{3}$ 1983;

Sekerak and Arai, 1977;

Margolis and Arthur, $1979^{3}$

Sekerak, 1975;

Love and Moser, 1976, ${ }^{3}$ 1983;

Sekerak and Arai, 1977;

Margolis and Arthur, $1979^{3}$

Sekerak, 1975;

Love and Moser, 1976, ${ }^{3}$ 19833;

Sekerak and Arai, 1977;

Margolis and Arthur, 19793;

Gibson, 19963; Love et al. $2002^{3}$

Sekerak, 1975;

Love and Moser, 1976, ${ }^{3}$ 1983';

Sekerak and Arai, 1977;

Margolis and Arthur, 19793;

Gibson, 19963; Love et al. $2002^{3}$

Sekerak, 1975;

Love and Moser, 1976, ${ }^{3}$ 1983';

Sekerak and Arai, 1977;

Margolis and Arthur, 1979;

Gibson, $1996^{3}$

Sekerak and Arai, 1977;

Margolis and Arthur, $1979^{3}$ 
Table 1. Continued

\begin{tabular}{|c|c|c|c|}
\hline Species $^{2}$ & Infection Site & Locality & References \\
\hline $\begin{array}{l}\text { D—Lecithochirium exodicum } \\
\text { McFarlane, } 1936 \text { (Syn. Sterrhurus } \\
\text { exodicus [McFarlane, 1936] } \\
\text { Yamaguti, 1958) }\end{array}$ & Stomach & $\begin{array}{l}\text { Off British Columbian } \\
\text { coast, northern Pacific } \\
\text { Ocean }\end{array}$ & $\begin{array}{l}\text { Sekerak, 1975; } \\
\text { Love and Moser, 1976,3 19833; } \\
\text { Sekerak and Arai, 1977; } \\
\text { Margolis and Arthur, 19793; } \\
\text { Gibson, 19963; Love et al. 2002³ }\end{array}$ \\
\hline $\begin{array}{l}\text { D-Opechona occidentalis } \\
\text { Montgomery, } 1957\end{array}$ & Intestine & $\begin{array}{l}\text { Off British Columbian } \\
\text { coast, Northern Pacific } \\
\text { Ocean }\end{array}$ & $\begin{array}{l}\text { Arai, 19693; } \\
\text { Love and Moser, 1976, }{ }^{3} 1983^{3} ; \\
\text { Sekerak and Arai, 1977; } \\
\text { Margolis and Arthur, 19793; } \\
\text { Bray and Gibson, 1990; } \\
\text { Gibson, 19963; Love et al. } 2002^{3}\end{array}$ \\
\hline D—Podocotyle araii Gibson, 1986 & Intestine & $\begin{array}{l}\text { Off British Columbian } \\
\text { coast }\end{array}$ & Gibson, $1996^{3,4}$; Love et al. $2002^{3}$ \\
\hline $\begin{array}{l}\text { D-Podocotyle radifistuli (Acena, 1941) } \\
\text { Gibson and Bray, } 1984 \\
\text { (Syn. Opecoelina radifistuli } \\
\text { [Acena, 1941] Yamaguti, 1958) }\end{array}$ & Intestine & $\begin{array}{l}\text { Off Pacific coast of USA, } \\
\text { Friday Harbor, WA }\end{array}$ & $\begin{array}{l}\text { Acena, 1941; Yamaguti, 1958, }{ }^{3} 1971^{3} ; \\
\text { Pratt and McCauley, 19613; } \\
\text { Love and Moser, 1976, }{ }^{3} 1983^{3} ; \\
\text { Love et al. } 2002^{3}\end{array}$ \\
\hline D—Podocotyle sp. & Intestine, pyloric caecum & $\begin{array}{l}\text { Northeastern Pacific } \\
\text { Ocean }\end{array}$ & $\begin{array}{l}\text { Sekerak, 1975; } \\
\text { Love and Moser, 1976, }{ }^{3} 1983^{3} ; \\
\text { Sekerak and Arai, 19774; } \\
\text { Margolis and Arthur, } 1979^{3}\end{array}$ \\
\hline $\begin{array}{l}\text { D-Prosorhynchus crucibulum } \\
\text { (Rudolphi, 1819) Odhner, } 1905\end{array}$ & Intestine, pyloric caecum & $\begin{array}{l}\text { Northeastern Pacific } \\
\text { Ocean }\end{array}$ & $\begin{array}{l}\text { Sekerak, 1975; } \\
\text { Love and Moser, 1976, }{ }^{3} 1983^{3} ; \\
\text { Sekerak and Arai, 19775; } \\
\text { Margolis and Arthur, } 1979^{3}\end{array}$ \\
\hline D_Prosorhynchus sp. & GI tract & $\begin{array}{l}\text { Off British Columbian } \\
\text { coast, northern Pacific } \\
\text { Ocean }\end{array}$ & $\begin{array}{l}\text { Gibson, } 1996^{3,5} ; \\
\text { Love et al. } 2002^{3}\end{array}$ \\
\hline $\begin{array}{l}\text { D-Steringophorus furciger } \\
\text { (Olsson, 1868) Odhner, } 1905 \\
\text { (Syn. Fellodistomum furcigerum } \\
\text { [Olsson, 1868] Yamaguti, 1953) }\end{array}$ & Bile duct, gall bladder & Northern Pacific Ocean & Love et al. $2002^{3}$ \\
\hline D-Digenea gen. sp. (undetermined) & - & Pacific Ocean off Canada & Arai, 19693; Margolis and Arthur, $1979^{3}$ \\
\hline M-Microcotyle sebastis Goto, 1894 & Gills & $\begin{array}{l}\text { Off San Pedro, California, } \\
\text { British Columbia, } \\
\text { Northern Pacific Ocean; } \\
\text { Off NW coast of Baja CA, } \\
\text { Mexico, between } \\
\text { Coronado Island and } \\
\text { San Quintin Bay }\end{array}$ & $\begin{array}{l}\text { Arai, 19693; Crane, 1972; } \\
\text { Love and Moser, 1976, }{ }^{3} \text { 1983; } \\
\text { Sekerak and Arai, 1977; } \\
\text { Margolis and Arthur, 19793; } \\
\text { Alvarado-Villamar and } \\
\text { Ruiz-Campos, 1992; } \\
\text { Love et al. 2002 }\end{array}$ \\
\hline M-Trochopus sp. & Gills & Northern Pacific Ocean & $\begin{array}{l}\text { Sekerak, 1975; } \\
\text { Love and Moser, 1976, }{ }^{3} \text { 1983; } \\
\text { Sekerak and Arai, 1977; } \\
\text { Margolis and Arthur, 19793; } \\
\text { Love et al. } 2002^{3}\end{array}$ \\
\hline MY_Kudoa clupeidae (Hahn, 1977) & Muscles & Off southern California & $\begin{array}{l}\text { Love and Moser, 1976, }{ }^{3} \text { 19833; } \\
\text { Moser et al. } 1976\end{array}$ \\
\hline
\end{tabular}


Table 1. Continued

\begin{tabular}{|c|c|c|c|}
\hline Species $^{2}$ & Infection Site & Locality & References \\
\hline $\mathrm{N}$-Anisakine nematodes & Body cavity, mesenteries ${ }^{6}$ & Off coast of Washington & Stern et al. 1976 \\
\hline $\mathrm{N}$-Anisakis sp. larva & $\begin{array}{l}\text { Body cavity, mesenteries, } \\
\text { wall of internal organs }\end{array}$ & $\begin{array}{l}\text { Off NW coast of Baja CA, } \\
\text { Mexico, between Coronado } \\
\text { Island and San Quintin Bay; } \\
\text { northern Pacific Ocean }\end{array}$ & $\begin{array}{l}\text { Dailey et al. 1980; } \\
\text { Love and Moser, } 1983^{3} ; \\
\text { Alvarado-Villamar and } \\
\text { Ruiz-Campos, 1992; Love et al. } 2002^{3}\end{array}$ \\
\hline $\mathrm{N}$-Ascarophis sebastodis Olsen, 1952 & Intestine & Off British Columbian coast & $\begin{array}{l}\text { Arai, } 1969^{3} ; \\
\text { Love and Moser, 1976, }{ }^{3} 1983^{3} ; \\
\text { Sekerak and Arai, 1977; } \\
\text { Margolis and Arthur, 19793; } \\
\text { Love et al. } 2002^{3}\end{array}$ \\
\hline $\mathrm{N}$-Contracaecum sp. larva & $\begin{array}{l}\text { Intestine, mesenteries, } \\
\text { stomach, stomach wall }\end{array}$ & $\begin{array}{l}\text { Northern Pacific Ocean; } \\
\text { Off NW coast of Baja CA, } \\
\text { Mexico, between Coronado } \\
\text { Island and San Quintin Bay }\end{array}$ & $\begin{array}{l}\text { Sekerak, 1975; } \\
\text { Love and Moser, 1976, }{ }^{3} 1983^{3} ; \\
\text { Sekerak and Arai, 1977; } \\
\text { Margolis and Arthur, 19793; } \\
\text { Alvarado-Villamar and } \\
\text { Ruiz-Campos, 1992; Love et al. } 2002^{3}\end{array}$ \\
\hline $\begin{array}{l}\text { N-Hysterothylacium aduncum } \\
\text { (Rudolphi, 1802) (Syn. } \\
\text { Thynnascaris adunca/um Rudolphi, ?) }\end{array}$ & Intestine, stomach & Northeastern Pacific Ocean & $\begin{array}{l}\text { Sekerak, 1975; } \\
\text { Love and Moser, 1976, }{ }^{3} 1983^{3} ; \\
\text { Sekerak and Arai, 1977; } \\
\text { Margolis and Arthur, 1979; } \\
\text { Love et al. } 2002^{3}\end{array}$ \\
\hline
\end{tabular}

Sebastes rubrivinctus (flag rockfish)

\begin{tabular}{|c|c|c|c|}
\hline $\begin{array}{l}\text { CO_Chondracanthus pinguis } \\
\text { Wilson, } 1912\end{array}$ & Gill cavity & North Pacific Ocean & Kazachenko, 1986 \\
\hline CO_Clavella parva Wilson, 1912 & Fins & North Pacific Ocean & Kazachenko, 1986 \\
\hline $\begin{array}{l}\text { CO_Lepeophtheirus parviventris } \\
\text { Wilson, } 1905\end{array}$ & Body surface, fins & $\begin{array}{l}\text { Off California, } \\
\text { North Pacific Ocean }\end{array}$ & $\begin{array}{l}\text { Wilson, } 1908^{3} ; \text { Yamaguti, } 1963^{3} ; \\
\text { Kabata, } 1973^{3} ; \\
\text { Love and Moser, } 1976{ }^{3}{ }^{3} 1983^{3}\end{array}$ \\
\hline $\begin{array}{l}\text { CO_Lepeophtheirus salmonis } \\
\text { salmonis (Krǿyer, 1837) } \\
\text { (Alt. Rep. Lepeophtheirus salmonis } \\
\text { [Krǿyer, 1837]) }\end{array}$ & Surface of fish & Off US Pacific coast & $\begin{array}{l}\text { Kazachenko et al. 1972; } \\
\text { Love and Moser, 1976, }{ }^{3} 1983^{3}\end{array}$ \\
\hline $\begin{array}{l}\text { CO_Naobranchia occidentalis } \\
\text { Wilson, } 1915\end{array}$ & Gills & North Pacific Ocean & Kazachenko, 1986 \\
\hline $\begin{array}{l}\text { CO_Parabrachiella robusta } \\
\text { (Wilson, 1912) (Syns. Brachiella } \\
\text { robusta [Wilson, 1912]; } \\
\text { Neobrachiella robusta } \\
\text { [Wilson, 1912]) }\end{array}$ & Gills & $\begin{array}{l}\text { Off west coast of } \\
\text { Vancouver Island, } \\
\text { British Columbia }\end{array}$ & $\begin{array}{l}\text { Kabata, } 1970^{7} ; \\
\text { Love and Moser, 1976, } 1983^{3,7} ; \\
\text { Margolis and Arthur, } 1979^{3}\end{array}$ \\
\hline CO_Procolobomatus kyphosus & Cephalic sensory canals & North Pacific Ocean & Kazachenko, 1986 \\
\hline
\end{tabular}

(Sekerak, 1970) (Syn. Colobomatus kyphosus Sekerak, 1970) 
Table 1. Continued

\begin{tabular}{|c|c|c|c|}
\hline Species ${ }^{2}$ & Infection Site & Locality & References \\
\hline $\begin{array}{l}\text { CO_Pseudopeniculus asinus } \\
\text { (Kabata and Wilkes, 1977) } \\
\text { (Syn. Peniculus asinus Kabata } \\
\text { and Wilkes, 1977) }\end{array}$ & Fins & North Pacific Ocean & Kazachenko, 1986 \\
\hline $\begin{array}{l}\text { M-Allobenedenia sebastodi } \\
\text { (Egorova, 1994) Yang, Kritsky } \\
\text { and Sun, } 2004 \text { (Syn. Megalocotyloides } \\
\text { sebastodi Egorova, 1994) }\end{array}$ & Gills & Western Pacific Ocean & Egorova, 1994a, 1994b \\
\hline $\begin{array}{l}\text { M-Megalocotyle marginata Folda, } \\
1928 \text { (Syn. Trochopus marginata } \\
\text { [Folda, 1928] Price, 1936) }\end{array}$ & Gills & Off Pacific coast of USA & Love et al. $2002^{3}$ \\
\hline $\begin{array}{l}\text { MY_Henneguya sebasta Moser } \\
\text { and Love, } 1975\end{array}$ & Heart & Off California & $\begin{array}{l}\text { Jensen, } 1977^{\circ} ; \\
\text { Love and Moser, } 1983^{3}\end{array}$ \\
\hline $\mathrm{N}$-Anisakis sp. larva & $\begin{array}{l}\text { Body cavity, viscera, } \\
\text { musculature }\end{array}$ & $\begin{array}{l}\text { Off southern California, } \\
\text { Pacific coast of USA }\end{array}$ & $\begin{array}{l}\text { Love and Moser, } 1983^{3} ; \\
\text { Dailey et al. 1980; Love et al. } 2002^{3}\end{array}$ \\
\hline $\begin{array}{l}\text { N-Hysterothylacium aduncum } \\
\text { (Rudolphi, 1802) }\end{array}$ & Intestine & Off Pacific coast of USA & Love et al. $2002^{3}$ \\
\hline $\mathrm{N}$-Thynnascaris sp. & Intestine, stomach & Off southern California & $\begin{array}{l}\text { Jensen, } 1975,{ }^{9} 1977^{8} \\
\text { Love and Moser, } 1976,{ }^{3} 1983^{3}\end{array}$ \\
\hline
\end{tabular}

1 Based on Froese and Pauly (2019), the following host synonymies also were searched for S. elongatus: Sebastodes elongatus (Ayres, 1859) and for S. rubrivinctus: Sebastichthys rubrivinctus Jordan and Gilbert, 1880 and Sebastodes rubrivinctus (Jordan and Gilbert, 1880).

2 A-Acanthocephala; B-Bacteria; C-Cestoda; CO—Copepoda; D-Digenea; M-Monogenea; MY-Myxosporida; N-Nematoda. We used the taxonomic designation and authority for each species provided by World Register of Marine Species (WoRMS) (www.marinespecies.org). 3 These references are host-parasite checklists and/or comprehensive reviews of host group(s) and should not be considered original records. 4 Gibson (1996, p. 184) stated that the "Podocotyle sp." of Sekerak and Arai (1977) from S. elongatus is Podocotyle araii.

5 Gibson (1996, pp. 46-47) considers "Prosorhynchus crucibulum (Rudolphi, 1819)" of Sekerak and Arai (1977) from S. elongatus to be an unidentified species of Prosorhynchus Odhner, 1905 (i.e., Prosorhynchus sp.).

6 Record for this parasite species had no information for this characteristic, so the most likely infection site is given.

7 Kabata (1970, pp. 875-882) noted Brachiella robusta from S. rubrivinctus as a "Morphological type" or "form" of this species, Brachiella robusta cf. longidigita, which he listed as "B. robusta f. longidigita," and this was noted by Love and Moser (1976, p. 307). Love and Moser (1983, p. 355) later listed this species as Neobrachiella robusta noting that Kabata (1970) had reported it as "Brachiella r." This species is now recognized as Parabrachiella robusta (see World Register of Marine Species [WoRMS] at www.marinespecies.org).

8 See Love and Moser (1983, pp. 355, 571).

9 See Love and Moser (1976, pp. 307, 476).

and McCauley, 1964, 1965; McCauley, 1964, 1968). Digeneans were stained with either Mayer's carmalum or Van Cleave's hematoxylin combination and mounted in Canada balsam (see McCauley and Pequegnat, 1968). Drawings were done with the aid of an Olympus $\mathrm{CH} 30$ compound microscope using a drawing tube and a Nikon SuperhighPerformance 3 Zoom Coolpix 990 digital camera and image software system. Measurements are in micrometers $(\mu \mathrm{m})$ with the holotype followed by the range and mean in parentheses; the number [n] of measurements is also noted where needed. Two-dimensional measurements are given with the length before the width. Comparative measurements were taken from the original species descriptions or redescriptions unless otherwise stated. If needed, some critical measurements that either were not available or were obviously in error in the original descriptions were calculated from original illustrations and are identified herein. Fish classification and authorities follow FishBase (Froese and Pauly, 2019) while digenean authorities follow Bray (2008b) and WoRMS (2020b). Digenean identification was based on Bray (2008a, 2008b) and ecological terms followed Bush et al. (1997). 
While we understand that in situ fixation of digeneans is not ideal, specimens measured herein appeared in good condition upon close examination. Furthermore, we felt that because of the inherent rarity of this material (i.e., from the deep sea) the benefits to our limited knowledge of deep-sea helminth communities afforded by this study justified the completion of this work.

Digenean specimens used in this study are housed in the Harold W. Manter Laboratory of Parasitology (HWML), University of Nebraska-Lincoln, Lincoln, Nebraska (original catalog numbers HWML 42810 and HWML 42863). The abbreviation (USNM-formerly USNPC) represents the United States National Parasite Collection, Smithsonian National Museum of Natural History (NMNH), Washington, DC, USA.

\section{Results}

Table 1 provides a list of the parasites reported from $\mathrm{Se}$. elongatus and Se. rubrivinctus, and as can be seen, there are a good variety of them. To date, at least 14 species of parasites have been found in the flag rockfish, Se. rubrivinctus, including eight copepod, two monogenean, and one myxosporidan species as well as nematodes from three genera; no digeneans are known from this host. There are at least twice that number (28) of parasite species known from the greenstriped rockfish, Se. elongatus. Table 1 documents representatives from one acanthocephalan and one bacteria genus, two cestodes, three copepods, 13 digeneans, two monogeneans, one myxosporidan, and five taxa of nematodes. Among the digenetic trematodes, representatives of seven families including the Bucephalidae Poche, 1907; Derogenidae Nicoll, 1910; Fellodistomidae Nicoll, 1909; Hemiuridae Looss, 1899; Lepocreadiidae Odhner, 1905; Opecoelidae Ozaki, 1925; and the Zoogonidae were known from this host prior to this study.

Discovered was a new species of Steganoderma parasitizing Se. elongatus and an unidentified, though damaged, congeneric specimen infecting Se. rubrivinctus; both are described here.

Class: Trematoda Rudolphi, 1808

Subclass: Digenea Carus, 1863

Order: Plagiorchiida La Rue, 1957

Family: Zoogonidae Odhner, 1902

Syns: Cephaloporidae Yamaguti, 1934; Dupliciporiidae Reimer, 1985; Steganodermatidae Yamaguti, 1934.
Subfamily: Lepidophyllinae Stossich, 1904 Syns: Hudsoniinae Campbell, 1975; Lecithostaphylinae Odhner, 1911; Pseudopalaeorchiinae Yamaguti, 1971; Steganodermatinae Yamaguti, 1934; Steganoderminae Yamaguti, 1934.

Genus: Steganoderma Stafford, 1904

Syn: Nordosstrema Issaitschikov, 1928.

\section{Steganoderma eamiqtrema $\mathrm{n} . \mathrm{sp}$.}

(Figs. 1-3)

Description: Measurements based on 6 adult, wholemounted specimens with very light stain; 1 adult specimen rolled with disrupted vitelline fields; 1 adult specimen with black markings (perhaps due to burning during staining process) and partially full of air (perhaps due to air entering specimen during mounting process). Measurements, ratios, and proportions given in Table 2. With the characteristics of the genus. Body elongate oval to spindle shaped, attenuated at both ends, widest in middle third of body, flattened dorsoventrally. Forebody attenuated to rounded extremity; hindbody attenuated to truncate or curved extremity. Tegument spined; spines easily dislodged, larger and denser anteriorly, become smaller and more sporadic at level of posterior region of middle third of body and extend almost to posterior extremity. Pre-oral lobe absent. Oral sucker distinct, subglobular or round, mouth either terminal $[n=3]$ or subterminal [ $n=3]$. Ventral sucker small, indistinct, sessile, median but can appear submedian sinistral when worm rolled, round or subcircular, larger than oral sucker, at junction of anterior and middle thirds of body. Prepharynx absent. Pharynx small, muscular, round to dolioform. Esophagus straight to slightly sinuous, moderately long and longer than pharynx. Granular parenchymal cells around pharynx and esophagus, inconspicuous in smaller specimens. Intestinal bifurcation in or around level of mid-forebody, often overlapped by cirrus pouch. Ceca moderately wide, anterior portion distinct, posterior portion indistinct, extend posteriorly near lateral margins to or near level of testes, arcuate posteriorly and terminate blindly.

Testes 2, smooth to slightly indented, globular to oval, opposite, near junction of middle and posterior thirds of body, immediately posterior to vitelline fields and anterior margins often overlapped by latter; 1 specimen with very oblong oval right testis; 1 specimen with contiguous testes (i.e., no inter-testicular region) due to rolling. Cirrus pouch large, voluminous, distinct, thick-walled, clavate to comma shaped, filled with prostatic cells, extends posterio-dextrally along transverse course from genital pore at or near 


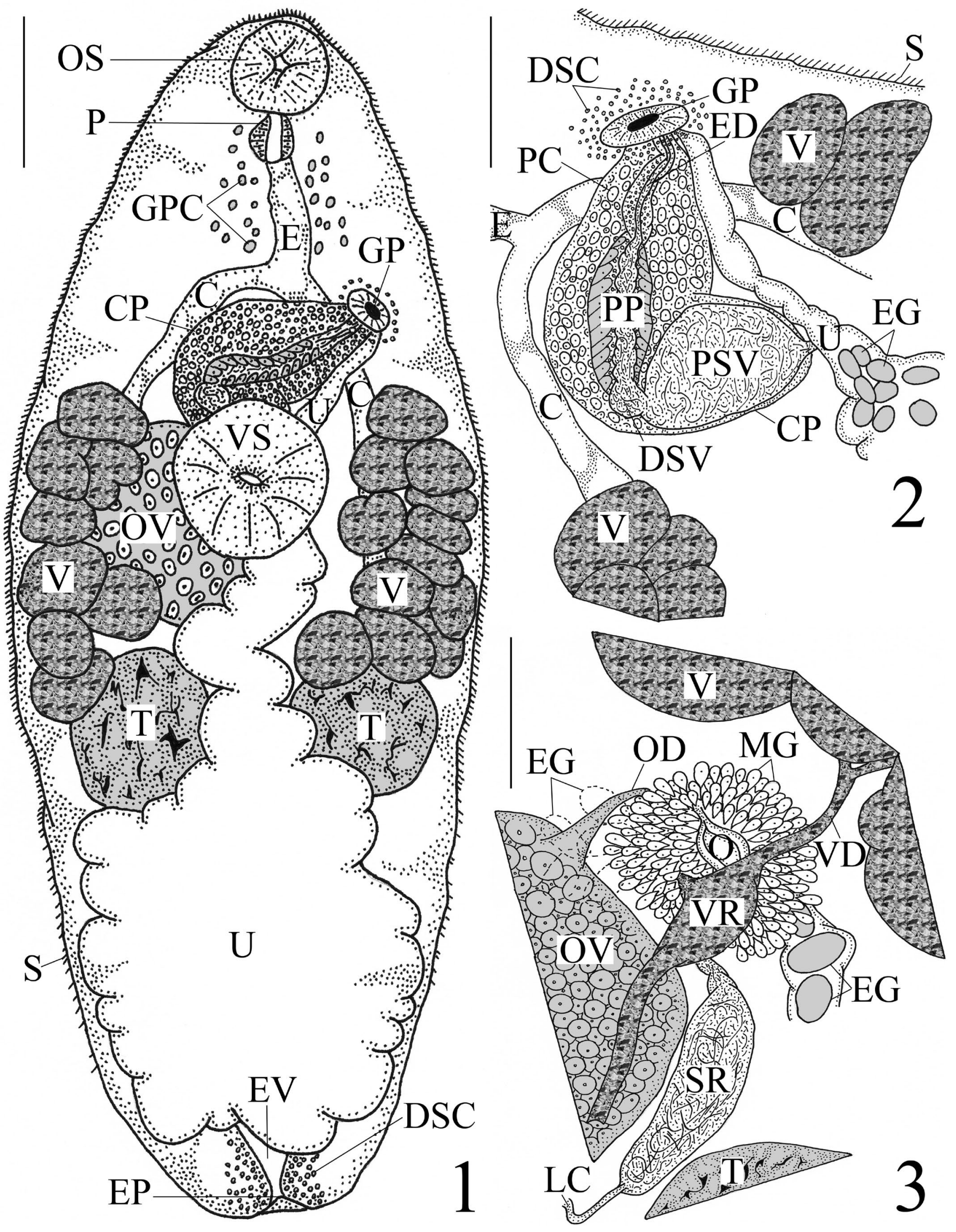


Figures 1-3. Steganoderma eamiqtrema n. sp. (Digenea: Zoogonidae: Lepidophyllinae) from the intestine of the greenstriped rockfish, Sebastes elongatus Ayres, 1859 (Scorpaeniformes: Sebastidae). 1. Composite of adult, ventral view. 2. Male terminal genitalia, ventral view. 3. Composite of proximal female system, ventral view. Note: Vitelline system illustrated ventral for ease of observation; exact details of main vitelline duct and Laurer's canal/seminal receptacle connections to oviduct obscured by Mehlis' gland and egg-filled uterus. Abbreviations: C, Cecum; CP, Cirrus pouch; DSC, Dark-staining cells; DSV, Distal seminal vesicle; $E_{\text {, }}$ Esophagus; ED, Ejaculatory duct; EG, Egg; EP, Excretory pore; EV, Excretory vesicle; GP, Genital pore; GPC, Granular parenchymal cells; LC, Laurer's canal; MG, Mehlis' gland; O, Oötype; OD, Oviduct; OS, Oral sucker; OV, Ovary; P, Pharynx; PC, Prostatic cells; PP, Pars prostatica; PSV, Proximal seminal vesicle; S, Spines; SR, Seminal receptacle; T, Testis; U, Uterus; V, Vitellarium; VD, Vitelline duct; VR, vitelline reservoir; VS, Ventral sucker. Scale bars: Figs. 1, $2=165 \mu \mathrm{m}$; Fig. $3=60 \mu \mathrm{m}$.

Table 2. Measurements, morphometric percentages, and morphometric ratios of Steganoderma eamiqtrema $\mathrm{n}$. sp. from present study compared to recognized species of Steganoderma Stafford, 1904

\begin{tabular}{|c|c|c|c|c|c|c|c|c|c|}
\hline Parasite & $\begin{array}{c}\text { S. eamiqtrema } \\
\text { n. sp. }\end{array}$ & $\begin{array}{c}\text { S. atherinae } \\
\text { (Price, 1934) } \\
\text { Manter, } \\
1947\end{array}$ & $\begin{array}{l}\text { S. formosum } \\
\text { Stafford, } \\
1904\end{array}$ & $\begin{array}{l}\text { S. formosum } \\
\text { Stafford, } \\
1904\end{array}$ & $\begin{array}{l}\text { S. macro- } \\
\text { phallus } \\
\text { Szidat and } \\
\text { Nani, } 1951\end{array}$ & $\begin{array}{l}\text { S. oviformis } \\
\text { Szidat, } \\
1962\end{array}$ & $\begin{array}{l}\text { S. rhiphidium } \\
\text { Wang, } \\
1986\end{array}$ & $\begin{array}{c}\text { S. szidati } \\
\text { Viozzi, Flores } \\
\text { and Ostrowski } \\
\text { de Núñez, } \\
2000\end{array}$ & $\begin{array}{l}\text { S. valchetensis } \\
\text { Etchegoin, } \\
\text { Cremonte } \\
\text { and Escalante, } \\
2002\end{array}$ \\
\hline Reference & $\begin{array}{l}\text { Present } \\
\text { study } \\
\text { (Figs. 1-3) }\end{array}$ & $\begin{array}{l}\text { Price (1934, } \\
\text { fig. 3) }\end{array}$ & $\begin{array}{c}\text { Stafford } \\
(1904)^{6}\end{array}$ & $\begin{array}{c}\text { Bray (1979, } \\
\text { 1987, fig. } \\
12 A)^{6}\end{array}$ & $\begin{array}{l}\text { Szidat and } \\
\text { Nani (1951, } \\
\text { fig. 5) }\end{array}$ & $\begin{array}{l}\text { Szidat (1962, } \\
\text { fig. 1) }\end{array}$ & $\begin{array}{l}\text { Wang (1986, } \\
\text { fig. 6) }\end{array}$ & $\begin{array}{l}\text { Viozzi et al. } \\
(2000 \text {, fig. 1) }\end{array}$ & $\begin{array}{l}\text { Etchegoin } \\
\text { et al. (2002, } \\
\text { figs. } 1-3)\end{array}$ \\
\hline $\mathrm{n}=$ & 6 & $\geq 3^{7}$ & 1 & 1 & $\geq 2^{7}$ & 5 & 1 & 35 & 10 \\
\hline Host(s) & $\begin{array}{c}\text { Sebastes } \\
\text { elongatus } \\
\text { Ayres, } 1859 \\
\text { (Sebastidae) }\end{array}$ & $\begin{array}{c}\text { Hypoatherina } \\
\text { harrington- } \\
\text { ensis (Goode, } \\
1877 \text { ) } \\
\text { (Atherinidae) }\end{array}$ & $\begin{array}{l}\text { Hippoglossus } \\
\text { hippoglossus } \\
\text { (Linnaeus, } \\
\text { 1758) (Pleuro- } \\
\text { nectidae) }\end{array}$ & $\begin{array}{l}\text { Hippoglossus } \\
\text { hippoglossus } \\
\text { (Linnaeus, } \\
\text { 1758) (Pleuro- } \\
\text { nectidae) }\end{array}$ & $\begin{array}{l}\text { Basilichthys } \\
\text { microlepid- } \\
\text { otus (Jenyns, } \\
\text { 1841); } \\
\text { Odontesthes } \\
\text { smitti (Lahille, } \\
\text { 1929) (Ather- } \\
\text { inopsidae) }\end{array}$ & $\begin{array}{c}\text { Aplochiton } \\
\text { zebra } \\
\text { Jenyns, } 1842 \\
\text { (Galaxiidae) }\end{array}$ & $\begin{array}{l}\text { Paralichthys } \\
\text { olivaceus } \\
\text { (Temminck } \\
\text { and Schlegel, } \\
\text { 1846) (Paral- } \\
\text { ichthyidae) }\end{array}$ & $\begin{array}{c}\text { Galaxias } \\
\text { maculatus } \\
\text { (Jenyns, 1842); } \\
\text { Galaxias } \\
\text { platei } \\
\text { Steindachner, } \\
1898 \\
\text { (Galaxiidae) }\end{array}$ & $\begin{array}{c}\text { Gymno- } \\
\text { characinus } \\
\text { bergi } \\
\text { Steindach- } \\
\text { ner, } 1903 \\
\text { (Characidae) }\end{array}$ \\
\hline Locality & $\begin{array}{l}\text { Off Newport, } \\
\text { Oregon }\end{array}$ & $\begin{array}{c}\text { Samaná Bay, } \\
\text { Dominican } \\
\text { Republic }\end{array}$ & $\begin{array}{l}\text { Off east } \\
\text { coast of } \\
\text { Canada }\end{array}$ & $\begin{array}{l}\text { Off east } \\
\text { coast of } \\
\text { Canada }\end{array}$ & $\begin{array}{l}\text { Limay and } \\
\text { Quequén } \\
\text { Grande Rivers, } \\
\text { Argentina }\end{array}$ & $\begin{array}{l}\text { Patagonia, } \\
\text { Argentina }\end{array}$ & $\begin{array}{l}\text { Off Pingtan } \\
\text { County, Fujian } \\
\text { Province, } \\
\text { China }\end{array}$ & $\begin{array}{c}\text { Lake } \\
\text { Gutiérrez } \\
\text { and other } \\
\text { glacial lakes, } \\
\text { Andean } \\
\text { Patagonia, } \\
\text { Argentina }\end{array}$ & $\begin{array}{c}\text { Valcheta Creek, } \\
\text { northern } \\
\text { Patagonia, } \\
\text { Argentina }\end{array}$ \\
\hline Length & $\begin{array}{c}1,280 \\
(1,140-1,840 \\
1,453)^{1}\end{array}$ & $\begin{array}{c}1,220- \\
1,360\end{array}$ & 2,600 & $2,772^{4}$ & $700-800$ & $1,900-2,300$ & 2,560 & $\begin{array}{c}(822-1,870 \\
1,141)\end{array}$ & $\begin{array}{c}(400-645 ; \\
523)\end{array}$ \\
\hline $\begin{array}{l}\text { Width at } \\
\text { pharynx }\end{array}$ & $\begin{array}{c}424 \\
(320-424 ; \\
384)\end{array}$ & $211^{4}$ & $-^{3}$ & $457^{4}$ & $\begin{array}{l}(198-203 ; \\
201)^{4}\end{array}$ & $\begin{array}{c}(820-836 \\
828) \\
{[n=2]^{1,4}}\end{array}$ & $445^{4}$ & $\begin{array}{c}347 \\
{[n=1]^{1,4}}\end{array}$ & $\begin{array}{c}223 \\
{[n=1]^{1,4}}\end{array}$ \\
\hline Width at VS1 & $\begin{array}{c}656 \\
(584-720 \\
647)\end{array}$ & $425-544$ & 810 & $707^{4}$ & 280 & $1,480-1,600$ & $742^{4}$ & $\begin{array}{c}(397-963 ; \\
569)\end{array}$ & $\begin{array}{c}(168-392 ; \\
278)\end{array}$ \\
\hline Width at $\mathrm{T}^{1}$ & $\begin{array}{c}672 \\
(528-672 ; \\
629)\end{array}$ & $330^{4}$ & $-^{3}$ & $652^{4}$ & $\begin{array}{l}(230-257 \\
244)^{4}\end{array}$ & $\begin{array}{c}(1,339-1,672 \\
1,506) \\
{[n=2]^{1,4}}\end{array}$ & 1,260 & $\begin{array}{c}579 \\
{[n=1]^{1,4}}\end{array}$ & $\begin{array}{c}281 \\
{[n=1]^{1,4}}\end{array}$ \\
\hline Forebody $L^{1}$ & $\begin{array}{c}416 \\
(388-608 \\
489)\end{array}$ & $425-510$ & $-^{3}$ & $989^{4}$ & $\begin{array}{l}(225-248 ; \\
237)^{4}\end{array}$ & $\begin{array}{c}(583-669 ; \\
626) \\
{[n=2]^{1,4}}\end{array}$ & $806^{4}$ & $\begin{array}{l}(219-495 \\
333)\end{array}$ & $\begin{array}{c}(99-199 \\
145)\end{array}$ \\
\hline Hindbody L & $\begin{array}{c}696 \\
(624-1,280 \\
857)\end{array}$ & $768^{4}$ & -3 & $1,587^{4}$ & $\begin{array}{l}(306-396 \\
351)^{4}\end{array}$ & $\begin{array}{c}(820-1,045 \\
933) \\
{[n=2]^{1,4}}\end{array}$ & $1,537^{4}$ & $\begin{array}{c}(400-1,095 \\
602)\end{array}$ & $\begin{array}{c}(98-210 \\
154)\end{array}$ \\
\hline $\begin{array}{l}\text { Oral sucker } \\
\text { (OS) L }\end{array}$ & $\begin{array}{c}124 \\
(120-160 \\
138)\end{array}$ & $152-160$ & $-^{3}$ & $152^{4}$ & $\begin{array}{c}(108-113 \\
111)^{4}\end{array}$ & $\begin{array}{c}(216-230 \\
223) \\
{[n=2]^{1,4}}\end{array}$ & 167 & $\begin{array}{c}(95-238 \\
155)\end{array}$ & $\begin{array}{c}(90-147 \\
119)\end{array}$ \\
\hline OS $W^{1}$ & $\begin{array}{c}152 \\
(140-160 ; \\
151)\end{array}$ & $100-133$ & -3 & $185^{4}$ & 120 & $200-250$ & 192 & $\begin{array}{c}(95-248 \\
156)\end{array}$ & $\begin{array}{c}(80-155 \\
127)\end{array}$ \\
\hline Prepharynx L & $0(0 ; 0)$ & $20-40$ & $-^{3}$ & $0^{4}$ & $0^{4}$ & 0 & $0^{4}$ & $\begin{array}{c}0 \text { [extremely } \\
\text { short or absent] }\end{array}$ & 0 \\
\hline Pharynx L & $56(56-76 ; 63)$ & $80-88$ & $-^{3}$ & $76^{4}$ & 60 & $\begin{array}{c}(64-125 ; 95) \\
{[n=2]^{1,4}}\end{array}$ & 144 & $(41-83 ; 63)$ & $(33-65 ; 48)$ \\
\hline Pharynx W & $60(56-68 ; 61)$ & 60 & $-^{3}$ & $54^{4}$ & 50 & $100-150$ & 112 & $(36-95 ; 66)$ & $(32-42 ; 37)$ \\
\hline Esophagus L & $\begin{array}{c}164(92-232 ; \\
162)\end{array}$ & $\begin{array}{l}\sim 0 \text { [very short } \\
\text { or absent] }\end{array}$ & -3 & $348^{4}$ & $\begin{array}{c}(36-45 \\
41)^{4}\end{array}$ & $\begin{array}{c}(65-167 ; 116) \\
{[\mathrm{n}=2]^{1,4}}\end{array}$ & 160 & $32[n=1]^{1,4}$ & $8[n=1]^{1,4}$ \\
\hline
\end{tabular}


Table 2. Continued

\begin{tabular}{|c|c|c|c|c|c|c|c|c|c|}
\hline Parasite & $\begin{array}{c}\text { S. eamiqtrema } \\
\text { n. sp. }\end{array}$ & $\begin{array}{c}\text { S. atherinae } \\
\text { (Price, 1934) } \\
\text { Manter, } \\
1947\end{array}$ & $\begin{array}{l}\text { S. formosum } \\
\text { Stafford, } \\
1904\end{array}$ & $\begin{array}{l}\text { S. formosum } \\
\text { Stafford, } \\
1904\end{array}$ & $\begin{array}{l}\text { S. macro- } \\
\text { phallus } \\
\text { Szidat and } \\
\text { Nani, } 1951\end{array}$ & $\begin{array}{l}\text { S. oviformis } \\
\text { Szidat, } \\
1962\end{array}$ & $\begin{array}{c}\text { S. rhiphidium } \\
\text { Wang, } \\
1986\end{array}$ & $\begin{array}{c}\text { S. szidati } S \\
\text { Viozzi, Flores } \\
\text { and Ostrowski } \\
\text { de Núñez, a } \\
2000\end{array}$ & $\begin{array}{l}\text { S. valchetensis } \\
\text { Etchegoin, } \\
\text { Cremonte } \\
\text { and Escalante, } \\
2002\end{array}$ \\
\hline $\begin{array}{l}\text { Intestinal } \\
\text { bifurc. } \\
\text { anterior } \\
\text { to VS }\end{array}$ & $\begin{array}{c}76 \\
(60-248 \\
145) \\
{[n=5]^{1}}\end{array}$ & $165^{4}$ & $-^{3}$ & $413^{4}$ & $\begin{array}{l}(31-59 ; \\
45)^{4}\end{array}$ & $\begin{array}{c}(172-251 ; \\
212) \\
{[n=2]^{1,4}}\end{array}$ & $265^{4}$ & $\begin{array}{c}105 \\
{[n=1]^{1,4}}\end{array}$ & $\begin{array}{c}38 \\
{[n=1]^{1,4}}\end{array}$ \\
\hline $\begin{array}{c}\text { Post-cecal } \\
\text { region L }\end{array}$ & $\begin{array}{c}\overline{-}^{3} \\
788-920 \\
{[n=3]^{1}}\end{array}$ & $-^{3}$ & $-^{3}$ & $1,228^{4}$ & $\begin{array}{c}392 \\
{[n=1]^{1,4}}\end{array}$ & $\begin{array}{c}(691-899 \\
795) \\
{[n=2]^{1.4}}\end{array}$ & $1,346^{4}$ & $\begin{array}{c}558 \\
{[n=1]^{1,4}}\end{array}$ & $\begin{array}{c}200 \\
{[n=1]^{1,4}}\end{array}$ \\
\hline VS L & $\begin{array}{c}164(144-236 \\
189)\end{array}$ & $80-100$ & $-^{3}$ & $217^{4}$ & $(126 ; 126)^{4}$ & $550-650$ & 312 & $\begin{array}{c}(162-362 ; \\
234)\end{array}$ & $\begin{array}{c}(90-199 ; \\
145)\end{array}$ \\
\hline VS W & $\begin{array}{c}164(148-236 \\
191)\end{array}$ & $108-120$ & $-^{3}$ & $174^{4}$ & 130 & $610-820$ & 320 & $\begin{array}{l}(162-409 ; \\
250)\end{array}$ & $\begin{array}{c}(88-210 ; \\
154)\end{array}$ \\
\hline $\mathrm{DT}^{1} \mathrm{~L}$ & $\begin{array}{c}200(188-268 ; \\
213)\end{array}$ & $\begin{array}{c}120-160 \\
\text { [both testes] }\end{array}$ & $-^{3}$ & $348^{4}$ & $\begin{array}{c}(99-112 \\
106)^{4}\end{array}$ & $\begin{array}{c}(271-324 ; \\
298)[n=2]^{1,4}\end{array}$ & 272 & $(131-309 ; 191)$ & $(46-88 ; 71)$ \\
\hline DT W & $\begin{array}{c}240(196-248 ; \\
231)\end{array}$ & $\begin{array}{c}60-80 \\
\text { [both testes] }\end{array}$ & $-^{3}$ & $261^{4}$ & $(58-72 ; 65)^{4}$ & $\begin{array}{c}(237-272 ; 255) \\
{[\mathrm{n}=2]^{1,4}}\end{array}$ & $128\{244\}^{1,4}$ & $(107-302 ; 175)$ & $(41-67 ; 54)$ \\
\hline$S T^{1} \mathrm{~L}$ & $\begin{array}{c}184(180-244 \\
201)\end{array}$ & & -3 & $304^{4}$ & $(76-108 ; 92)^{4}$ & $\begin{array}{c}(259-272 ; 266) \\
{[n=2]^{1,4}}\end{array}$ & $192\{329\}^{1,4}$ & $(98-321 ; 195)$ & $(52-90 ; 65)$ \\
\hline ST W & $\begin{array}{c}216(192-252 ; \\
216)\end{array}$ & & $---^{3}$ & $228^{4}$ & $(72-76 ; 74)^{4}$ & $\begin{array}{c}(194-293 ; 244) \\
{[\mathrm{n}=2]^{1,4}}\end{array}$ & 194 & $(107-309 ; 163)$ & $(40-72 ; 54)$ \\
\hline $\begin{array}{l}\text { Inter-testicular } \\
\text { region } \\
\text { (ITR) W }\end{array}$ & $\begin{array}{c}108 \\
(16-140 \\
98)[n=5]^{1}\end{array}$ & $93^{4}$ & $-^{3}$ & $109^{4}$ & $(58-81 ; 70)^{4}$ & $\begin{array}{c}(376-540 ; 458) \\
{[n=2]^{1,4}}\end{array}$ & $424^{4}$ & $132[\mathrm{n}=1]^{1,4}$ & $108[n=1]^{1,4}$ \\
\hline $\begin{array}{l}\text { Post-testicular } \\
\text { region } \\
\text { (PTR) L }\end{array}$ & $\begin{array}{c}400 \\
(400-664 ; \\
505)\end{array}$ & $412^{4}$ & $-^{3}$ & $924^{4}$ & $\begin{array}{c}(256-324 ; \\
290)^{4}\end{array}$ & $\begin{array}{c}(432-836 ; 634) \\
{[\mathrm{n}=2]^{1,4}}\end{array}$ & $827^{4}$ & $474[n=1]^{1,4}$ & $150[n=1]^{1,4}$ \\
\hline $\begin{array}{l}\text { PTR W at } \\
\text { mid-point }\end{array}$ & $\begin{array}{c}528 \\
(400-552 ; \\
496)\end{array}$ & $206^{4}$ & -3 & $554^{4}$ & $\begin{array}{l}(144-171 ; \\
158)^{4}\end{array}$ & $\begin{array}{c}(972-1,379 ; \\
1,176)[n=2]^{1,4}\end{array}$ & $1,134^{4}$ & $421[n=1]^{1,4}$ & $192[\mathrm{n}=1]^{1,4}$ \\
\hline $\begin{array}{l}\text { Cirrus pouch } \\
\text { (CP) } L\end{array}$ & $\begin{array}{c}576 \\
(260-576 ; \\
429)[n=5]^{1}\end{array}$ & $120-160$ & $-^{3}$ & $587^{4}$ & $\begin{array}{l}(211-248 ; \\
230)^{4}\end{array}$ & $\begin{array}{c}(561-690 ; 626) \\
{[n=2]^{1,4}}\end{array}$ & $318^{4}$ & $(248-571 ; 354)$ & $(118-178 ; 145)$ \\
\hline CP W & $\begin{array}{l}116(96-188 ; \\
150)[\mathrm{n}=5]^{1}\end{array}$ & $60-80$ & $-^{3}$ & $130^{4}$ & $(58-68 ; 63)^{4}$ & $\begin{array}{c}(194-230 ; 212) \\
{[\mathrm{n}=2]^{1,4}}\end{array}$ & $106^{4}$ & $(67-162 ; 109)$ & $(30-50 ; 38)$ \\
\hline $\begin{array}{l}\text { Seminal } \\
\text { vesicle (SV) } \\
\text { - proximal } \\
\text { portion L }\end{array}$ & $\begin{array}{c}188 \\
(50-188 \\
152) \\
{[n=4]^{1}}\end{array}$ & $82^{4}$ [entire] & $-^{3}$ & $109^{4}$ [entire] & $\begin{array}{c}131[n=1]^{1,4} \\
\text { [entire] }\end{array}$ & $108[n=1]^{1,4}$ & $159^{4}$ [entire] & $\begin{array}{c}(81-226 ; 133) \\
{[\text { [entire] }} \\
\{42-163 ; 103\} \\
{[n=3]^{1,4}} \\
\text { [prox. portion] }\end{array}$ & $\begin{array}{c}(31 ; 31) \\
{[\mathrm{n}=2]^{1,4}}\end{array}$ \\
\hline $\begin{array}{l}\text { SV_proximal } \\
\text { portion W }\end{array}$ & $\begin{array}{c}106 \\
(40-156 ; 108) \\
{[n=4]^{1}}\end{array}$ & $26^{4}$ [entire] & $-^{3}$ & $\begin{array}{c}87^{4} \\
\text { [entire] }\end{array}$ & $\begin{array}{c}45 \\
{[n=1]^{1,4}} \\
{[\text { entire] }}\end{array}$ & $\begin{array}{c}130 \\
{[n=1]^{1,4}}\end{array}$ & $\begin{array}{c}74^{4} \\
\text { [entire] }\end{array}$ & $\begin{array}{c}(60-119 ; 80) \\
{[\text { entire] }} \\
\{47-79 ; 65\} \\
{[\mathrm{n}=3]^{1,4}} \\
\text { [prox. portion] }\end{array}$ & $\begin{array}{l}(28-31 ; 30) \\
{[\mathrm{n}=2]^{1,4}}\end{array}$ \\
\hline $\begin{array}{l}\text { SV_distal } \\
\text { portion L }\end{array}$ & $\begin{array}{c}86 \\
(20-86 ; 56) \\
{[n=4]^{1}}\end{array}$ & & $-^{3}$ & & & $216[n=1]^{1,4}$ & & $\begin{array}{c}\{37-63 ; 54\} \\
{[n=3]^{1,4}} \\
{[\text { distal portion] }}\end{array}$ & $\begin{array}{l}(23-27 ; 25) \\
{[\mathrm{n}=2]^{1,4}}\end{array}$ \\
\hline $\begin{array}{l}\text { SV_distal } \\
\text { portion W }\end{array}$ & $\begin{array}{c}68 \\
(24-68 ; 47) \\
{[n=4]^{1}}\end{array}$ & & $-^{3}$ & & & $65[n=1]^{1,4}$ & & $\begin{array}{c}\{42-53 ; 46\} \\
{[n=3]^{1,4}} \\
{[\text { distal portion] }}\end{array}$ & $\begin{array}{c}(28-31 ; 30) \\
{[n=2]^{1,4}}\end{array}$ \\
\hline $\begin{array}{l}\text { Pars } \\
\text { prostatica } \\
\text { (PP) L }\end{array}$ & $\begin{array}{c}180 \\
(126-194 ; 166) \\
{[n=4]^{1}}\end{array}$ & $-^{3}$ & $-^{3}$ & $239^{4}$ & $\begin{array}{c}90 \\
{[n=1]^{1,4}}\end{array}$ & $-^{3}$ & $-^{3}$ & $\begin{array}{c}(68-74 ; 71) \\
{[\mathrm{n}=2]^{1,4}}\end{array}$ & $\begin{array}{l}(46-61 ; 54) \\
{[n=2]^{1,4}}\end{array}$ \\
\hline PP W & $\begin{array}{c}56(38-62 ; 54) \\
{[\mathrm{n}=4]^{1}}\end{array}$ & $-^{3}$ & $-^{3}$ & $33^{4}$ & $23[n=1]^{1,4}$ & $-^{3}$ & $-^{3}$ & $\begin{array}{c}(37-42 ; 40) \\
{[n=2]^{1,4}}\end{array}$ & $\begin{array}{l}(23-27 ; 25) \\
{[\mathrm{n}=2]^{1,4}}\end{array}$ \\
\hline Ejac. duct L & $\begin{array}{c}-3(60-128 ; 95) \\
{[n=3]^{1}}\end{array}$ & -3 & -3 & $163^{4}$ & $\begin{array}{l}36 \\
{[n=1]^{1,4}}\end{array}$ & $\begin{array}{c}151 \\
{[n=1]^{1,4}}\end{array}$ & $148^{4}$ & $\begin{array}{c}(12-71 ; 35) \\
\{126-142 ; \\
134\}[n=2]^{1,4} \\
{[\text { with cirrus] }}\end{array}$ & $\begin{array}{c}(57-65 ; 61) \\
{[\mathrm{n}=2]^{1,4}}\end{array}$ \\
\hline Ejac. duct W & $\overline{1}^{3}(12-16 ;$ & $-^{3}$ & -3 & $33^{4}$ & $\begin{array}{c}5 \\
{[n=1]^{1,4}}\end{array}$ & $\begin{array}{c}32 \\
{[n=1]^{1,4}}\end{array}$ & $21^{4}$ & $\begin{array}{l}(26-41 ; 35) \\
\text { [with cirrus] }\end{array}$ & $\begin{array}{l}(2-4 ; 3) \\
{[n=2]^{1,4}}\end{array}$ \\
\hline $\begin{array}{l}\text { Genital pore } \\
\text { (GP) to lateral } \\
\text { margin }\end{array}$ & $\begin{array}{c}76 \\
(72-256 \\
116)\end{array}$ & $41^{4}$ & $-^{3}$ & $65^{4}$ & $(31-36 ; 34)^{4}$ & $\begin{array}{c}(86-146 ; 116) \\
{[n=2]^{1,4}}\end{array}$ & $32^{4}$ & $\begin{array}{c}0 \\
\text { [marginal] }\end{array}$ & {$\left[\begin{array}{c}23 \\
{[n=1]^{1,4}}\end{array}\right.$} \\
\hline $\begin{array}{l}\text { GP to anterior } \\
\text { end }\end{array}$ & $\begin{array}{c}368 \\
(296-464 ; 385)\end{array}$ & $320-400$ & -3 & $772^{4}$ & $\begin{array}{c}(171-176 \\
174)^{4}\end{array}$ & $\begin{array}{c}(302-376 ; 339) \\
{[n=2]^{1,4}}\end{array}$ & $647^{4}$ & $\begin{array}{c}279 \\
{[n=1]^{1,4}}\end{array}$ & $\begin{array}{c}158 \\
{[n=1]^{1,4}}\end{array}$ \\
\hline
\end{tabular}


Table 2. Continued

\begin{tabular}{|c|c|c|c|c|c|c|c|c|c|}
\hline Parasite & $\begin{array}{l}\text { S. eamiqtrema } \\
\text { n. sp. }\end{array}$ & $\begin{array}{c}\text { S. atherinae } \\
\text { (Price, 1934) } \\
\text { Manter, } \\
1947\end{array}$ & $\begin{array}{l}\text { S. formosum } \\
\text { Stafford, } \\
1904\end{array}$ & $\begin{array}{l}\text { S. formosum } \\
\text { Stafford, } \\
1904\end{array}$ & $\begin{array}{l}\text { S. macro- } \\
\text { phallus } \\
\text { Szidat and } \\
\text { Nani, } 1951\end{array}$ & $\begin{array}{l}\text { S. oviformis } \\
\text { Szidat, } \\
1962\end{array}$ & $\begin{array}{c}\text { S. rhiphidium } \\
\text { Wang, } \\
1986\end{array}$ & $\begin{array}{c}\text { S. szidati } \\
\text { Viozzi, Flores } \\
\text { and Ostrowski } \\
\text { de Núñez, } \\
2000 \\
\end{array}$ & $\begin{array}{l}\text { S. valchetensis } \\
\text { Etchegoin, } \\
\text { Cremonte } \\
\text { and Escalante, } \\
2002\end{array}$ \\
\hline $\begin{array}{l}\text { Pre-ovarian } \\
\text { region L }\end{array}$ & $\begin{array}{c}552 \\
(440-656 ; 551)\end{array}$ & $521^{4}$ & $-^{3}$ & $1,044^{4}$ & $\begin{array}{c}(315-320 \\
318)^{4}\end{array}$ & $\begin{array}{c}(1,058-1,191 ; \\
1,125)[\mathrm{n}=2]^{1,4}\end{array}$ & $1,240^{4}$ & $\begin{array}{c}542 \\
{[n=1]^{1,4}}\end{array}$ & $\begin{array}{c}288 \\
{[n=1]^{1,4}}\end{array}$ \\
\hline Ovary (OV) L & $\begin{array}{c}160(148-268 ; \\
192)\end{array}$ & $60-120$ & $-^{3}$ & $261^{4}$ & $(45-77 ; 61)^{4}$ & $\begin{array}{c}(216-230 ; 223) \\
{[\mathrm{n}=2]^{1,4}}\end{array}$ & $\begin{array}{c}240 \\
\{191\}^{1,4}\end{array}$ & $(71-202 ; 143)$ & $(32-56 ; 47)$ \\
\hline OV W & $\begin{array}{c}168(160-304 ; \\
204)\end{array}$ & $88-120$ & $-^{3}$ & $261^{4}$ & $(45-77 ; 61)^{4}$ & $\begin{array}{c}(172-230 ; 201) \\
{[\mathrm{n}=2]^{1,4}}\end{array}$ & $\begin{array}{c}160 \\
\{191\}^{1,4}\end{array}$ & $(83-250 ; 139)$ & $(31-61 ; 41)$ \\
\hline VS to OV & $\begin{array}{c}72 \\
{[n=1]^{1,5}(0 ; 0)} \\
{[n=5]^{1,5}}\end{array}$ & $0^{4}$ & $-^{3}$ & $\begin{array}{c}0^{4} \\
\text { [VS overlaps } \\
\text { OV] }\end{array}$ & $\begin{array}{l}(0 ; 0)^{4} \\
{[\text { VS overlaps }} \\
\text { OV] }\end{array}$ & $\begin{array}{c}(0-86 ; 43) \\
{[n=2]^{1,4}} \\
\text { [VS overlaps OV } \\
\text { in } 1 \text { specimen] }\end{array}$ & $159^{4}$ & $\begin{array}{c}0 \\
{[n=1]^{1,4}} \\
\text { [VS overlaps } \\
\text { OV in fig 1a] }\end{array}$ & $\begin{array}{c}0 \\
{[n=1]^{1,4}} \\
{[\text { VS overlaps }} \\
\text { OV] }\end{array}$ \\
\hline OV to DT & $\begin{array}{c}36(20-76 ; 43) \\
{[n=5]^{1}}\end{array}$ & $180^{4}$ & -3 & $315^{4}$ & $(0-9 ; 5)^{4}$ & $\begin{array}{c}(0-21 ; 11) \\
{[n=2]^{1,4}}\end{array}$ & $180^{4}$ & $\begin{array}{c}0[\mathrm{n}=1]^{1,4} \\
\text { [OV overlaps } \\
\text { DT in fig. 1a] }\end{array}$ & $\begin{array}{c}4 \\
{[n=1]^{1,4}}\end{array}$ \\
\hline $\begin{array}{l}\text { Seminal } \\
\text { receptacle } \\
\text { (SR) L }\end{array}$ & $\begin{array}{c}200 \\
(100-200 \\
150)[\mathrm{n}=2]^{1}\end{array}$ & $46^{4}$ & $-^{3}$ & -3 & $-^{3}$ & $\begin{array}{c}(188-194 ; \\
191) \\
{[n=2]^{1,4}}\end{array}$ & $-^{3}$ & $\begin{array}{c}53 \\
{[n=1]^{1,4}}\end{array}$ & $(31-66 ; 50)$ \\
\hline SR W & $\begin{array}{c}120(80-120 ; 100) \\
{[\mathrm{n}=2]^{1}}\end{array}$ & $60\{36\}^{1,4}$ & $-^{3}$ & $-^{3}$ & $-^{3}$ & $\begin{array}{c}(86-125 ; 106) \\
{[n=2]^{1,4}}\end{array}$ & $-^{3}$ & $\begin{array}{c}42 \\
{[n=1]^{1,4}}\end{array}$ & $(23-46 ; 34)$ \\
\hline $\begin{array}{l}\text { No. of Vitelline } \\
\text { follicles (VF) } \\
\text { —dextral field }\end{array}$ & $\begin{array}{c}9 \\
(8-10 ; 9) \\
{[n=5]^{1}}\end{array}$ & Numerous $^{4}$ & $10-12$ & $9^{4}$ & $(9-10 ; 10)^{4}$ & $\begin{array}{c}(7-8 ; 8) \\
{[\mathrm{n}=2]^{1,4}}\end{array}$ & 10 & $(6-11 ; 9)$ & $(7-13 ; 10)$ \\
\hline $\begin{array}{l}\text { Dextral vitelline } \\
\text { field } L\end{array}$ & $\begin{array}{c}344 \\
(276-528 \\
378)[n=5]^{1}\end{array}$ & $320^{4}$ & $-^{3}$ & $674^{4}$ & $\begin{array}{l}(99-108 ; \\
104)^{4}\end{array}$ & $\begin{array}{c}(564-605 \\
585) \\
{[n=2]^{1,4}}\end{array}$ & $254^{4}$ & $\begin{array}{c}289 \\
{[n=1]^{1,4}}\end{array}$ & $\begin{array}{c}77 \\
{[n=1]^{1,4}}\end{array}$ \\
\hline $\begin{array}{l}\text { No. of VF- } \\
\text { sinistral field }\end{array}$ & $\begin{array}{c}12(12 ; 12) \\
{[n=5]^{1}}\end{array}$ & Numerous $^{4}$ & $10-12$ & $10^{4}$ & $(12-13 ; 13)^{4}$ & $\begin{array}{l}(12 ; 12) \\
{[n=2]^{1,4}}\end{array}$ & 10 & $(9-13 ; 11)$ & $(8-13 ; 10)$ \\
\hline $\begin{array}{l}\text { Sinistral } \\
\text { vitelline } \\
\text { field L }\end{array}$ & $\begin{array}{c}360 \\
(296-488 ; \\
382)[n=5]^{1}\end{array}$ & $314^{4}$ & $-^{3}$ & $641^{4}$ & $(90-99 ; 95)^{4}$ & $\begin{array}{c}(501-540 ; \\
521) \\
{[n=2]^{1,4}}\end{array}$ & $254^{4}$ & $\begin{array}{c}368 \\
{[n=1]^{1,4}}\end{array}$ & $\begin{array}{c}92 \\
{[n=1]^{1,4}}\end{array}$ \\
\hline $\begin{array}{l}\text { Dextral field } \\
\text { VF L }\end{array}$ & $\begin{array}{c}(88-100 ; 93) \\
{[\mathrm{n}=5]^{\prime}} \\
(68-148 ; 99) \\
{[\mathrm{n}=25]^{1}}\end{array}$ & $\begin{array}{c}(15-31 ; 27) \\
{[\mathrm{n}=5]^{1,4}}\end{array}$ & $-^{3}$ & $\begin{array}{c}(109-141 ; 125) \\
{[n=9]^{1.4}}\end{array}$ & $\begin{array}{l}(18-23 ; 20) \\
{[n=10]^{1,4}}\end{array}$ & $\begin{array}{c}(86-146 ; 119) \\
{[\mathrm{n}=10]^{1,4}}\end{array}$ & $\begin{array}{l}(53-74 ; 66) \\
{[n=10]^{1,4}}\end{array}$ & $(48-129 ; 75)$ & $\begin{array}{c}(19-27 ; 22) \\
{[\mathrm{n}=8]^{1,4}}\end{array}$ \\
\hline $\begin{array}{l}\text { Dextral field } \\
\text { VF W }\end{array}$ & $\begin{array}{c}(48-80 ; 62) \\
{[n=5] ;} \\
(36-120 ; 70) \\
{[n=25]^{1}}\end{array}$ & $\begin{array}{c}(10-15 ; 13) \\
{[n=5]^{1,4}}\end{array}$ & $-^{3}$ & $\begin{array}{c}(98-130 ; 117) \\
{[n=9]^{1,4}}\end{array}$ & $\begin{array}{l}(14-18 ; 16) \\
{[n=10]^{1,4}}\end{array}$ & $\begin{array}{l}(63-105 ; 79) \\
{[\mathrm{n}=10]^{1,4}}\end{array}$ & $\begin{array}{l}(53-74 ; 63) \\
{[n=10]^{1,4}}\end{array}$ & $(29-121 ; 63)$ & $\begin{array}{c}(15-19 ; 18) \\
{[\mathrm{n}=8]^{1,4}}\end{array}$ \\
\hline $\begin{array}{l}\text { Sinistral field } \\
\text { VF L }\end{array}$ & $\begin{array}{c}(68-100 ; 84) \\
{[n=5] ;} \\
(60-180 ; 104) \\
{[n=25]^{1}}\end{array}$ & $\begin{array}{c}(21-31 ; 24) \\
{[\mathrm{n}=5]^{1,4}}\end{array}$ & $-^{3}$ & $\begin{array}{c}(109-152 ; 133) \\
{[\mathrm{n}=10]^{1,4}}\end{array}$ & $\begin{array}{c}(14-23 ; 21) \\
{[\mathrm{n}=8]^{1,4}}\end{array}$ & $\begin{array}{l}(86-125 ; 108) \\
{[\mathrm{n}=10]^{1,4}}\end{array}$ & $\begin{array}{l}(53-74 ; 63) \\
{[n=10]^{1,4}}\end{array}$ & $(41-136 ; 73)$ & $\begin{array}{c}(19-35 ; 26) \\
{[\mathrm{n}=8]^{1,4}}\end{array}$ \\
\hline $\begin{array}{l}\text { Sinistral field } \\
\text { VF W }\end{array}$ & $\begin{array}{c}(48-64 ; 54) \\
{[n=5] ;} \\
(40-120 ; 68) \\
{[n=25]^{1}}\end{array}$ & $\begin{array}{c}(10-15 ; 14) \\
{[\mathrm{n}=5]^{1,4}}\end{array}$ & $-^{3}$ & $\begin{array}{c}(109-163 ; 122) \\
{[\mathrm{n}=10]^{1,4}}\end{array}$ & $\begin{array}{c}(14-18 ; 15) \\
{[\mathrm{n}=8]^{1,4}}\end{array}$ & $\begin{array}{l}(43-84 ; 57) \\
{[\mathrm{n}=10]^{1,4}}\end{array}$ & $\begin{array}{c}(53-74 ; 64) \\
{[n=10]^{1,4}}\end{array}$ & $(34-131 ; 65)$ & $\begin{array}{l}(15-27 ; 21) \\
{[n=8]^{1,4}}\end{array}$ \\
\hline $\begin{array}{l}\text { Pre-vitelline } \\
\text { region } \\
\text { (PreVR) L }\end{array}$ & $\begin{array}{c}424 \\
(376-576 ; \\
464)[n=5]^{1}\end{array}$ & $495^{4}$ & $-^{3}$ & $924^{4}$ & $\begin{array}{l}(225-230 ; \\
228)^{4}\end{array}$ & $\begin{array}{c}(604-711 ; 658) \\
{[\mathrm{n}=2]^{1,4}}\end{array}$ & $1,240^{4}$ & $\begin{array}{c}453 \\
{[n=1]^{1,4}}\end{array}$ & $\begin{array}{c}185 \\
{[n=1]^{1,4}}\end{array}$ \\
\hline $\begin{array}{l}\text { Post-vitelline } \\
\text { region } \\
\text { (PostVR) L }\end{array}$ & $\begin{array}{c}528 \\
(528-824 ; 646) \\
{[n=5]^{1}}\end{array}$ & $495^{4}$ & $-^{3}$ & $1,218^{4}$ & $\begin{array}{l}(342-410 ; \\
376)^{4}\end{array}$ & $\begin{array}{c}(712-1,087 \\
900)[n=2]^{i, 4}\end{array}$ & $\begin{array}{c}1,113^{4} \\
{[n=1]^{1,4}}\end{array}$ & $\begin{array}{c}532 \\
{[n=1]^{1,4}}\end{array}$ & 273 \\
\hline $\begin{array}{l}\text { Vitelline } \\
\text { reservoir L }\end{array}$ & $\begin{array}{c}80 \\
(34-80 ; 59) \\
{[n=3]^{1}}\end{array}$ & $-^{3}$ & $-^{3}$ & $-^{3}$ & $-^{3}$ & $\begin{array}{c}(105-108 ; 107) \\
{[\mathrm{n}=2]^{1,4}}\end{array}$ & $64^{4}$ & $-^{3}$ & $-^{3}$ \\
\hline $\begin{array}{l}\text { Vitelline } \\
\text { reservoir W }\end{array}$ & $\begin{array}{c}28 \\
(20-72 ; 40) \\
{[\mathrm{n}=3]^{1}}\end{array}$ & $-^{3}$ & $-^{3}$ & $-^{3}$ & $-^{3}$ & $\begin{array}{c}(42-108 ; 75) \\
{[\mathrm{n}=2]^{1,4}}\end{array}$ & $53^{4}$ & $-^{3}$ & $-^{3}$ \\
\hline Uterus L & $\begin{array}{l}880 \\
(696-1,400 ; 1,028)\end{array}$ & $933^{4}$ & -3 & $2,087^{4}$ & $\begin{array}{l}(382-432 ; \\
407)^{4}\end{array}$ & $\begin{array}{c}(1,598-1,860 ; \\
1,729)[\mathrm{n}=2]^{1,4}\end{array}$ & $2,152^{4}$ & $\begin{array}{c}953 \\
{[n=1]^{1,4}}\end{array}$ & $\begin{array}{c}423 \\
{[n=1]^{1,4}}\end{array}$ \\
\hline $\begin{array}{l}\text { Uterus } \mathrm{W} \text { at } \\
\text { widest point } \\
\text { in PTR }\end{array}$ & $\begin{array}{c}560 \\
(184-560 ; \\
459)[n=5]^{1}\end{array}$ & $253^{4}$ & $-^{3}$ & $522^{4}$ & $\begin{array}{c}(166-198 ; \\
182)^{4}\end{array}$ & $\begin{array}{c}(1,036-1,359 ; \\
1,198) \\
{[n=2]^{1,4}}\end{array}$ & $1,124^{4}$ & $\begin{array}{c}389 \\
{[n=1]^{1,4}}\end{array}$ & $\begin{array}{c}150 \\
{[\mathrm{n}=1]^{1,4}}\end{array}$ \\
\hline $\begin{array}{l}\text { Post-uterine } \\
\text { region } \\
\text { (PUR) L }\end{array}$ & $\begin{array}{c}72 \\
(72-248 ; 152) \\
{[\mathrm{n}=5]^{1}}\end{array}$ & $41^{4}$ & $-^{3}$ & $76^{4}$ & $\begin{array}{c}(135-140 ; \\
138)^{4}\end{array}$ & $\begin{array}{l}(21-84 ; 53) \\
{[\mathrm{n}=2]^{1,4}}\end{array}$ & $21^{4}$ & $\begin{array}{c}195 \\
{[n=1]^{1,4}}\end{array}$ & $\begin{array}{c}39 \\
{[n=1]^{1,4}}\end{array}$ \\
\hline
\end{tabular}


Table 2. Continued

\begin{tabular}{|c|c|c|c|c|c|c|c|c|c|}
\hline Parasite & $\begin{array}{c}\text { S. eamiqtrema } \\
\text { n. sp. }\end{array}$ & $\begin{array}{c}\text { S. atherinae } \\
\text { (Price, 1934) } \\
\text { Manter, } \\
1947\end{array}$ & $\begin{array}{l}\text { S. formosum } \\
\text { Stafford, } \\
1904\end{array}$ & $\begin{array}{l}\text { S. formosum } \\
\text { Stafford, } \\
1904\end{array}$ & $\begin{array}{l}\text { S. macro- } \\
\text { phallus } \\
\text { Szidat and } \\
\text { Nani, } 1951\end{array}$ & $\begin{array}{l}\text { S. oviformis } \\
\text { Szidat, } \\
1962\end{array}$ & $\begin{array}{c}\text { S. rhiphidium } \\
\text { Wang, } \\
1986\end{array}$ & $\begin{array}{c}\text { S. szidati } \\
\text { Viozzi, Flores } \\
\text { and Ostrowski } \\
\text { de Núñez, } \\
2000\end{array}$ & $\begin{array}{l}\text { S. valchetensis } \\
\text { Etchegoin, } \\
\text { Cremonte } \\
\text { and Escalante, } \\
2002\end{array}$ \\
\hline Egg L & $\begin{array}{c}(30-36 ; 34.0) \\
{[\mathrm{n}=5] ;} \\
(30-36 ; 33.7) \\
{[\mathrm{n}=22]^{1}}\end{array}$ & 32 & $-^{3}$ & $-^{3}$ & 34 & 40 & $45-54$ & $(26-36 ; 34)$ & $(28-34 ; 31)$ \\
\hline Egg W & $\begin{array}{c}(20-22 ; 20.8) \\
{[\mathrm{n}=5]^{\prime}} \\
(18-26 ; 21.0) \\
{[\mathrm{n}=23]^{1}}\end{array}$ & 18 & $-^{3}$ & $-^{3}$ & $18-20$ & 15 & $21-24$ & $(14-24 ; 18)$ & $(15-19 ; 17)$ \\
\hline Exc. vesicle $L$ & $\begin{array}{c}(336-1,056 ; 677) \\
{[n=3]^{3}}\end{array}$ & $-^{3}$ & $-^{3}$ & $-^{3}$ & $\begin{array}{l}(166-203 ; \\
185)^{4}\end{array}$ & -3 & $912^{4}$ & $-^{3}$ & $\begin{array}{c}119 \\
{[n=1]^{1,4}}\end{array}$ \\
\hline Exc. vesicle W & $\begin{array}{c}(140-200 ; 175) \\
{[\mathrm{n}=3]^{1}}\end{array}$ & $21^{4}$ & $-^{3}$ & $54^{4}$ & $\begin{array}{l}(81-104 \\
93)^{4}\end{array}$ & $\begin{array}{c}43 \\
{[n=1]^{1,4}}\end{array}$ & $32^{4}$ & $\begin{array}{c}100 \\
{[n=1]^{1,4}}\end{array}$ & $\begin{array}{c}35 \\
{[n=1]^{1,4}}\end{array}$ \\
\hline Forebody L \%² & $\begin{array}{c}32.5 \\
(29.7-37.8 ; 33.9)\end{array}$ & $34.8-37.5$ & $\sim 33.0+$ & $35.7^{4}$ & $\begin{array}{l}(31.0-32.1 \\
31.6)^{4}\end{array}$ & $\begin{array}{c}(29.1-30.7 \\
29.9)[n=2]^{1,4}\end{array}$ & 33.3 & $\begin{array}{c}49.0\{28.9\} \\
{[n=1]^{1,4}}\end{array}$ & 35.0 \\
\hline OS L \% ${ }^{2}$ & $\begin{array}{c}9.7 \\
(7.9-12.6 ; 9.7)\end{array}$ & $11.8-12.5$ & $-^{3}$ & $5.5^{4}$ & $\begin{array}{l}(14.1-15.4 \\
14.8)^{4}\end{array}$ & $\begin{array}{c}(10.0-11.4 \\
10.7)[n=2]^{1,4}\end{array}$ & 6.5 & $11.6-12.7$ & $22.5-22.8$ \\
\hline Pharynx L \% ${ }^{2}$ & $\begin{array}{c}4.4 \\
(3.8-5.1 ; 4.4)\end{array}$ & $6.5-6.6$ & $-^{3}$ & $2.7^{4}$ & $(7.5-8.6 ; 8.1)^{4}$ & $\begin{array}{c}(3.4-5.4 ; 4.4) \\
{[\mathrm{n}=2]^{1,4}}\end{array}$ & 5.6 & $4.4-5.0$ & $8.3-10.1$ \\
\hline $\begin{array}{l}\text { Esophagus } \\
\qquad \%^{2}\end{array}$ & $\begin{array}{c}12.8 \\
(7.4-16.1 ; 11.2)\end{array}$ & 0.0 & $-^{3}$ & $12.6^{4}$ & $(5.1-5.6 ; 5.4)^{4}$ & $\begin{array}{c}(3.4-7.3 ; 5.4) \\
{[\mathrm{n}=2]^{1,4}}\end{array}$ & 6.3 & $\begin{array}{c}2.4 \\
{[n=1]^{1,4}}\end{array}$ & $\begin{array}{c}1.5 \\
{[n=1]^{1,4}}\end{array}$ \\
\hline VS L $\%^{2}$ & $\begin{array}{c}12.8 \\
(10.6-20.7 ; 13.3)\end{array}$ & $6.6-7.4$ & $-^{3}$ & $7.8^{4}$ & $\begin{array}{c}(15.8-18.0 \\
16.9)^{4}\end{array}$ & $\begin{array}{c}(28.3-28.9 \\
28.6)\end{array}$ & 12.2 & $19.4-19.7$ & $22.5-30.9$ \\
\hline $\begin{array}{l}\text { Width at } \\
\text { VS } \%^{2}\end{array}$ & $\begin{array}{l}51.3 \\
(36.9-51.6 ; 45.6)\end{array}$ & $34.8-40.0$ & $-^{3}$ & $25.5^{4}$ & $\begin{array}{c}(35.0-40.0 \\
37.5)\end{array}$ & $\begin{array}{c}(69.6-77.9 \\
73.8)\end{array}$ & $29.0^{4}$ & $48.3-51.5$ & $42.0-60.8$ \\
\hline \multirow[t]{2}{*}{ CP L \% ${ }^{2}$} & $\begin{array}{c}45.0 \\
(21.0-45.0 ; 30.2)\end{array}$ & $9.8-11.8$ & $-^{3}$ & $21.2^{4}$ & $\begin{array}{c}(30.1-31.0 \\
30.6)^{4}\end{array}$ & $\begin{array}{c}(29.5-30.0 \\
29.8)[n=2]^{1,4}\end{array}$ & $12.4^{4}$ & $30.2-30.5$ & $27.6-29.5$ \\
\hline & {$[n=5]^{1}$} & & & & & & & & \\
\hline $\begin{array}{l}P P L \text { as \% } \\
\text { of CP L }\end{array}$ & $\begin{array}{c}31.3 \\
(31.3-48.5 \\
39.3)[\mathrm{n}=4]^{1}\end{array}$ & $-^{3}$ & $-^{3}$ & $40.7^{4}$ & $\begin{array}{c}36.3 \\
{[n=1]^{1,4}}\end{array}$ & $-^{3}$ & $-^{3}$ & $\begin{array}{c}(22.0-24.4 \\
23.2)[n=2]^{1,4}\end{array}$ & $\begin{array}{c}(25.4-32.9 \\
29.1)[n=2]^{1,4}\end{array}$ \\
\hline $\begin{array}{l}\text { Ejac. duct L } \\
\text { as \% of } \\
\text { CP L }\end{array}$ & $\begin{array}{c}\overline{3}^{3} \\
231.8-26.7 ; \\
23.9)[n=3]^{1}\end{array}$ & -3 & $-^{3}$ & $27.8^{4}$ & $\begin{array}{c}14.5 \\
{[n=1]^{1,4}}\end{array}$ & $\begin{array}{c}26.9 \\
{[n=1]^{1,4}}\end{array}$ & $46.5^{4}$ & $\begin{array}{c}4.8-12.4 \\
\{37.4-50.9 ; 44.2\} \\
{[n=2]^{1,4}} \\
{[\text { with cirrus] }}\end{array}$ & $\begin{array}{c}(30.7-36.0 \\
33.3) \\
{[n=2]^{1,4}}\end{array}$ \\
\hline DT L \%² & $\begin{array}{l}15.6 \\
(12.8-16.5 ; 14.9)\end{array}$ & $\begin{array}{c}9.8-11.8 \\
\text { [both testes] }\end{array}$ & $-^{3}$ & $12.6^{4}$ & $\begin{array}{l}(14.0-14.1 \\
14.1)^{4}\end{array}$ & $\begin{array}{c}(11.8-17.1 ; \\
14.5)[n=2]^{1,4}\end{array}$ & 10.6 & $15.9-16.5$ & $11.5-13.6$ \\
\hline ST L \% ${ }^{2}$ & $\begin{array}{l}14.4 \\
(12.5-16.5 ; 14.0)\end{array}$ & & $-^{3}$ & $11.0^{4}$ & $\begin{array}{c}(10.9-13.5 \\
12.2)^{4}\end{array}$ & $\begin{array}{c}(11.8-13.6 \\
12.7)[n=2]^{1,4}\end{array}$ & $7.5\{12.9\}^{1,4}$ & $11.9-17.2$ & $13.0-14.0$ \\
\hline $\begin{array}{l}\text { Width at } \\
\text { T \% }{ }^{2}\end{array}$ & $\begin{array}{l}52.5 \\
(36.5-52.5 ; 44.3)\end{array}$ & $25.2^{4}$ & -3 & $23.5^{4}$ & $\begin{array}{l}(32.1-32.9 ; \\
32.5)^{4}\end{array}$ & $\begin{array}{c}(70.5-72.7 \\
71.6)[\mathrm{n}=2]^{1,4}\end{array}$ & 49.2 & $\begin{array}{c}44.2 \\
{[n=1]^{1,4}}\end{array}$ & $\begin{array}{c}52.2 \\
{[n=1]^{1,4}}\end{array}$ \\
\hline OV L \% ${ }^{2}$ & $\begin{array}{l}12.5 \\
(10.3-14.6 ; 13.2)\end{array}$ & $4.9-8.8$ & $-^{3}$ & $9.4^{4}$ & $\begin{array}{l}(6.4-9.6 ; \\
8.0)^{4}\end{array}$ & $\begin{array}{c}(10.0-11.4 \\
10.7)[n=2]^{1,4}\end{array}$ & $9.4\{7.5\}^{1,4}$ & $8.6-10.8$ & $8.0-8.7$ \\
\hline VS to OV $\%^{2}$ & $\begin{array}{l}5.6 \\
{[n=1]^{1,5}} \\
(0.0 ; 0.0) \\
{[n=5]^{1,5}}\end{array}$ & 0.0 & $-^{3}$ & $0.0^{4}$ & $(0.0 ; 0.0)^{4}$ & $\begin{array}{c}(0.0-4.5 ; 2.3) \\
{[n=2]^{1,4}}\end{array}$ & $6.3\{7.5\}^{1,4}$ & $\begin{array}{l}0.0 \\
{[n=1]^{1,4}}\end{array}$ & $\begin{array}{l}0.0 \\
{[n=1]^{1,4}}\end{array}$ \\
\hline OV to DT $\%^{2}$ & $\begin{array}{c}2.8 \\
(1.8-4.3 ; 2.9) \\
{[n=5]^{1}}\end{array}$ & $13.8^{4}$ & $-^{3}$ & $11.4^{4}$ & $\begin{array}{l}(0.0-1.2 ; \\
0.6)^{4}\end{array}$ & $\begin{array}{c}(0.0-1.1 \\
0.6) \\
{[n=2]^{1,4}}\end{array}$ & $7.0^{4}$ & $\begin{array}{l}0.0 \\
{[n=1]^{1,4}}\end{array}$ & $\begin{array}{l}0.7 \\
{[n=1]^{1,4}}\end{array}$ \\
\hline $\begin{array}{l}\text { Dextral } \\
\text { vitelline } \\
\text { field } L \%^{2}\end{array}$ & $\begin{array}{c}26.9 \\
(24.2-28.7 \\
25.8)[n=5]^{1}\end{array}$ & $24.4^{4}$ & $-^{3}$ & $24.3^{4}$ & $\begin{array}{c}(13.5-14.1 ; \\
13.8)^{4}\end{array}$ & $\begin{array}{c}(24.5-31.8 \\
28.2) \\
{[n=2]^{1,4}}\end{array}$ & $9.9^{4}$ & $\begin{array}{c}22.0 \\
{[\mathrm{n}=1]^{1,4}}\end{array}$ & $\begin{array}{c}14.3 \\
{[n=1]^{1,4}}\end{array}$ \\
\hline $\begin{array}{l}\text { Sinistral } \\
\text { vitelline } \\
\text { field L \% }{ }^{2}\end{array}$ & $\begin{array}{c}28.1 \\
23.9-28.1 \\
(26.2)[n=5]^{1}\end{array}$ & $24.0^{4}$ & $-^{3}$ & $23.1^{4}$ & $\begin{array}{c}(12.4-12.9 ; \\
12.7)^{4}\end{array}$ & $\begin{array}{c}(21.8-28.4 \\
25.1) \\
{[\mathrm{n}=2]^{1,4}}\end{array}$ & $9.9^{4}$ & $\begin{array}{c}28.1 \\
{[n=1]^{1,4}}\end{array}$ & $\begin{array}{c}17.1 \\
{[n=1]^{1.4}}\end{array}$ \\
\hline PreVR L \%² & $\begin{array}{c}33.1 \\
(27.9-36.1 ; \\
32.3)[n=5]^{1}\end{array}$ & $37.8^{4}$ & $-^{3}$ & $33.3^{4}$ & $\begin{array}{l}(28.8-32.1 \\
30.5)^{4}\end{array}$ & $\begin{array}{c}(30.9-31.8 ; \\
31.4) \\
{[n=2]^{1,4}}\end{array}$ & $48.4^{4}$ & $\begin{array}{c}34.6 \\
{[n=1]^{1,4}}\end{array}$ & $\begin{array}{c}34.4 \\
{[n=1]^{1,4}}\end{array}$ \\
\hline PostVR L \% ${ }^{2}$ & $\begin{array}{c}41.3 \\
(41.3-47.0 \\
44.5)[n=5]^{1}\end{array}$ & $37.8^{4}$ & -3 & $43.9^{4}$ & $\begin{array}{l}(48.9-51.3 \\
50.1)^{4}\end{array}$ & $\begin{array}{c}(37.5-47.3 \\
42.4) \\
{[n=2]^{1,4}}\end{array}$ & $43.5^{4}$ & $\begin{array}{c}40.6 \\
{[n=1]^{1,4}}\end{array}$ & $\begin{array}{c}50.7 \\
{[n=1]^{1,4}}\end{array}$ \\
\hline PTR L \%² & $\begin{array}{c}31.3 \\
(31.3-37.3 ; 34.7)\end{array}$ & $\sim 33.0$ & $-^{3}$ & $33.3^{4}$ & $\begin{array}{l}(36.6-40.5 \\
38.6)^{4}\end{array}$ & $\begin{array}{c}(22.7-36.3 ; \\
29.5)[n=2]^{1,4}\end{array}$ & $32.3^{4}$ & $\begin{array}{c}36.2 \\
{[n=1]^{1,4}}\end{array}$ & $\begin{array}{c}27.9 \\
{[n=1]^{1,4}}\end{array}$ \\
\hline
\end{tabular}


Table 2. Continued

\begin{tabular}{|c|c|c|c|c|c|c|c|c|c|}
\hline Parasite & $\begin{array}{c}\text { S. eamiqtrema } \\
\text { n. sp. }\end{array}$ & $\begin{array}{c}\text { S. atherinae } \\
\text { (Price, 1934) } \\
\text { Manter, } \\
1947\end{array}$ & $\begin{array}{l}\text { S. formosum } \\
\text { Stafford, } \\
1904\end{array}$ & $\begin{array}{l}\text { S. formosum } \\
\text { Stafford, } \\
1904\end{array}$ & $\begin{array}{c}\text { S. macro- } \\
\text { phallus } \\
\text { Szidat and } \\
\text { Nani, } 1951\end{array}$ & $\begin{array}{l}\text { S. oviformis } \\
\text { Szidat, } \\
1962\end{array}$ & $\begin{array}{c}\text { S. rhiphidium } \\
\text { Wang, } \\
1986\end{array}$ & $\begin{array}{c}\text { S. szidati } \\
\text { Viozzi, Flores } \\
\text { and Ostrowski } \\
\text { de Núñez, } \\
2000 \\
\end{array}$ & $\begin{array}{l}\text { S. valchetensis } \\
\text { Etchegoin, } \\
\text { Cremonte } \\
\text { and Escalante, } \\
2002 \\
\end{array}$ \\
\hline PUR L \%² & $\begin{array}{l}5.6(5.6-21.8 \\
11.5)[\mathrm{n}=5]^{1}\end{array}$ & $3.1^{4}$ & $-^{3}$ & $2.7^{4}$ & $\begin{array}{c}(17.5-19.3 ; \\
18.4)^{4}\end{array}$ & $\begin{array}{c}(1.1-3.7 ; \\
2.4)[n=2]^{1,4}\end{array}$ & $0.8^{4}$ & $\begin{array}{c}14.9 \\
{[n=1]^{1,4}}\end{array}$ & $\begin{array}{c}7.2 \\
{[n=1]^{1,4}}\end{array}$ \\
\hline $\begin{array}{l}\text { OS:pharynx } \\
\text { width } \\
\text { ratio }\end{array}$ & $\begin{array}{c}1: 2.53 \\
(1: 2.26-2.67 \\
1: 2.48)\end{array}$ & $1: 1.67-2.22$ & $-^{3}$ & $1: 3.43^{4}$ & $1: 2.40$ & $\begin{array}{c}(1: 1.67-2.00 ; \\
1: 1.84)\end{array}$ & $1: 1.71$ & $1: 2.61-2.64$ & $1: 2.50-3.69$ \\
\hline $\begin{array}{l}\text { Sucker L } \\
\text { ratio }\end{array}$ & $\begin{array}{c}1: 1.32 \\
(1: 0.92-1.97 \\
1: 1.39)\end{array}$ & $1: 0.53-0.63$ & -3 & $1: 1.43^{4}$ & $\begin{array}{c}(1: 1.12-1.17 \\
1: 1.15)^{4}\end{array}$ & $\begin{array}{c}(1: 2.55-2.83 \\
1: 2.69) \\
{[n=2]^{1.4}}\end{array}$ & 1:1.87 & $1: 1.50$ & 1:1.00-1.35 \\
\hline $\begin{array}{c}\text { Sucker W } \\
\text { ratio }\end{array}$ & $\begin{array}{c}1: 1.08 \\
(1: 1.00-1.69 ; \\
1: 1.27)\end{array}$ & $1: 0.90-1.08$ & -3 & $1: 0.94^{4}$ & $1: 1.08$ & $\begin{array}{c}(1: 3.05-3.28 \\
1: 3.17)\end{array}$ & 1:1.67 & $1: 1.60$ & $\begin{array}{c}(1: 0.99-1.64 \\
1: 1.20)\end{array}$ \\
\hline
\end{tabular}

1 DT, dextral (right) testis; L, length; ST, sinistral (left) testis; T, testes; VS, ventral sucker; W, width; holotype followed by the range and mean in parentheses where applicable; number [n] of measurements provided if different from total number of worms examined; calculated values from figure(s) in \{ $\}$ if different from those given in description.

2 Proportion of body length.

3 -, this feature was either not observed, not measured, not described, and/or not illustrated.

4 This measurement was either not available or was in error in the original publication and calculated from original illustration(s).

5 Ventral sucker overlaps ovary in five specimens; only one specimen (holotype) has VS to OV distance $=72 \mu \mathrm{m}$ or $5.6 \%$ of body length.

6 As the type description of S. formosum by Stafford (1904) is rudimentary and lacks an illustration, we have used the information and morphological details of fig. 12A of Bray $(1979,1987)$ to represent this species (see also fig. 64.21 of Bray, 2008b; Table 3 of present study).

7 The reference did not give the number of specimens measured of this species. Price $(1934$, p. 4) indicated for S. atherinae that accession numbers were assigned for the holotype and for the paratypes (i.e., $\geq 2$ paratypes; $\Sigma=3+$ ). Szidat and Nani (1951, pp. 345-347) did not indicate the number of S. macrophallus collected or measured; however, they provided measurements for a few features (i.e., width of oral and ventral suckers, length and width of body, pharynx, and eggs) in one specimen. As they stated that S. macrophallus was found in silversides (locally called "pejerrey") of two species (Basilichthys microlepidotus [Jenyns, 1841] and Odontesthes smitti [Lahille, 1929] [Syn. Bachmannia smitti (Lahille, 1929)]) from the Limay and Quequén Rivers, Argentina, and the two worms illustrated in figs. 5a and 5b do not appear to be the same individual, the type description is based on at least 2 specimens.

level of intestinal bifurcation across left cecum and midline of worm to near posterior forebody before it curves back posterio-sinistrally to dorsally overlap anterior margin of ventral sucker and run to level at or near posterior margin of it. Seminal vesicle bipartite; proximal portion large, saccate to oblong oval; distal portion smaller than proximal portion, saccate, round; 1 specimen with very large, distorted, saccate proximal portion relative to distal portion, $160 \times 102$. Prostatic cells profuse within cirrus pouch, surround seminal vesicle with especially dense numbers of cells in mid to distal portion of cirrus pouch. Pars prostatica vesicular, cylindrical, conspicuous, rounded at both ends, occupies $1 / 3$ to $1 / 2$ of cirrus pouch length and filled with bleb-like cells; ejaculatory duct moderately long, narrow, tubular, occupies $1 / 5$ to $1 / 4$ of cirrus pouch length; cirrus present. Genital atrium fairly deep, round to oblong in shape with distinct thick-walled border, surrounded by dense number of dark-staining cells. Genital pore submedian, sinistral, nearer left margin than midline, anterior to ventral sucker and at level of intestinal bifurcation or just posterior to it.
Ovary large, smooth, globular to oval to subcircular, median to submedian dextral, partially overlapped by ventral sucker and either ventrally overlapped by vitelline fields or between both fields of follicles, pre-testicular and anterior to and almost contiguous with right testis, contiguous with or dorsally overlaps posterior margin of cirrus pouch; 1 specimen with subtriangularshaped ovary; 1 specimen with ovary to right of ventral sucker by 72 or $5.6 \%$ of body length. Seminal receptacle canalicular, often inconspicuous, elongate to teardrop-shaped, located between ovary and right testis and connects to oviduct from right side, extends to midline of worm and overlapped by uterine loops. Laurer's canal present, opening not observed but expected to be dorsal. Mehlis' gland large, conspicuous, median, either posterior to or immediately to left and at times contiguous with posterio-sinistral margin of ovary, proximate to vitelline reservoir. Uterus extensive, conspicuous, coiled, confined mostly to hindbody and occupies most of posttesticular region, extends almost to posterior extremity, proceeds anteriorly into inter-testicular region in a 
median narrow line then runs to left side of ovary and ventrally overlaps medial halves of testes before loops proceed anterio-sinistrally at level of ventral sucker to run to genital pore; 1 specimen without wide uterine loops in post-testicular region; 1 specimen with uterine loops that extend to posterior extremity (i.e., PUR $=0$ ). Metraterm present, inconspicuous due to light stain. Vitellaria in two conspicuous symmetrical fields of follicles along lateral margins in anterior hindbody, extend posteriorly from mid-level of cirrus pouch to level of ovary or anterior half of testes; follicles large, oblong to oval to globular in shape. Vitelline reservoir saccate to elongate, small, median, overlapped ventrally by uterus, left margin of ovary dorsally overlaps right margin of reservoir or latter located immediately sinistral to median/left margin of ovary. Paired vitelline ducts pass posterio-medially from vitelline fields near lateral margins to median vitelline reservoir; right duct passes medially and runs parallel to and dorsally overlaps posterio-sinistral margin of ovary; left duct passes sinistrally from median vitelline reservoir near level of posterior margin of ovary across uterine loops to median edge of sinistral vitelline field before it bifurcates into secondary anterior and posterior collecting ducts. Eggs numerous, oval, either smooth or crenulated, operculate, amber to light yellow, non-filamented, non-embryonated.

Excretory vesicle I-shaped, narrow at posterior end then noticeably expands in width after short distance anterior, conspicuous posteriorly, more inconspicuous anteriorly, extends anterior to at least level of ovary, often occluded by eggs in uterus; dense numbers of dark-staining cells surround posterior extent of vesicle. Excretory pore terminal.

Type host: Sebastes elongatus Ayres, 1859 (Syn. Sebastodes elongatus [Ayres, 1859]) (Scorpaeniformes: Sebastidae); greenstriped rockfish.

Type locality/collection date: Northeastern Pacific Ocean, approx. 40 km off Newport, Oregon, $44^{\circ} 38^{\prime} 15.5^{\prime \prime} \mathrm{N}$, $124^{\circ} 34^{\prime} 58.1^{\prime \prime} \mathrm{W}$; depth $=190 \mathrm{~m}$; 16-June-1964.

Site of infection: Intestine.

Deposited material: Holotype HWML 216295 (1 specimen on 1 slide); Paratypes HWML 42810 (5 specimens on 5 slides).

ZooBank registration: LSID urn:Isid:zoobank.org: act:6E3A5F1E-6BCC-47E3-8250-B5DD1D542D9B
Etymology: The species designation comes from a combination of "eamiq," the Arabic word for "deep," and the Greek "trema" for trematode; therefore, a species of "deepsea trematode."

Remarks: The present specimens belong within the Zoogonidae based on the following diagnostic combination of features: a genital pore in the forebody that is neither median nor close to the anterior extremity or lateral edge of the ventral sucker; a cirrus pouch with proximal extremity oriented posteriorly; an ovary that is entire and in the hindbody; a distinct alimentary tract as opposed to absent or greatly reduced; an ejaculatory duct and metraterm that are unarmed; testes in the hindbody; and a fish host (Bray, 2008a). Unlike members of the Cephaloporinae, Yamaguti, 1934, these specimens lack an unusually posterior ventral sucker, a lateral genital pore close to the level of the oral sucker, and a monacanthid host, yet they possess an operculate egg and vitellaria in paired fields of follicles and not in one or two compact masses (i.e., Zoogoninae Odhner, 1902); therefore, they are placed within the Lepidophyllinae (Bray, 2008b; Cutmore et al., 2014). This material belongs within Steganoderma because of its possessing an elongate oval to fusiform body, more or less entire testes and ovary, narrow ceca that extend to or near the level of the testes, an undivided sessile round ventral sucker with a mid-ventral aperture, vitelline fields in the hindbody, non-filamented eggs, neither enlarged circumoral spines nor pockets in the ejaculatory duct and metraterm, a claviform cirrus pouch, a saccular seminal vesicle (though bipartite, both portions are saccate), and these specimens infect the intestine instead of the urinary bladder of its fish host (Bray, 2008b).

Prior to this study, Steganoderma contained seven accepted species (WoRMS, 2020b; Blend et al., 2020); three of these are found in marine waters (S. atherinae, S. formosum, S. rhiphidium) while four species inhabit freshwater localities (S. macrophallus, S. oviformis, S. szidati, S. valchetensis). We noted numerous differences between S. eamiqtrema $\mathrm{n}$. sp. and the seven other species of Steganoderma (see Table 2) aside from the obvious dissimilarity in habitat and hosts (marine vs. freshwater) for the four freshwater species and the differing localities reported for the three other marine species (Samaná Bay, near Santa Barbara de Samaná, Dominican Republic, in the NW Atlantic [S. atherinae]; off the coast of the USA and Canada in the NW Atlantic and in the Barents Sea [S. formosum; see Table 3]; and off Fujian Province, China, in the NW Pacific [S. rhiphidium]). In overall size and measurements of features (Table 2), S. atherinae, S. macrophallus, S. szidati, and S. valchet- 
Table 3. Measurements, morphometric percentages, and morphometric ratios of Steganoderma sp. from present study and Steganoderma formosum Stafford, 1904 from the original description as well as from various redescriptions and supplemental descriptions (see Bray, 1987, p. 110)

\begin{tabular}{|c|c|c|c|c|c|c|c|c|}
\hline Parasite & $\begin{array}{c}\text { teganoderma } \\
\text { sp. }\end{array}$ & $\begin{array}{l}\text { S. formosum } \\
\text { Stafford, } 1904\end{array}$ & $\begin{array}{l}\text { S. formosum } \\
\text { Stafford, } 1904\end{array}$ & $\begin{array}{l}\text { S. formosum } \\
\text { Stafford, } 1904\end{array}$ & $\begin{array}{l}\text { S. formosum } \\
\text { Stafford, } 1904\end{array}$ & $\begin{array}{l}\text { S. formosum } \\
\text { Stafford, } 1904\end{array}$ & $\begin{array}{l}\text { S. formosum } \\
\text { Stafford, } 1904\end{array}$ & $\begin{array}{l}\text { S. formosum } \\
\text { Stafford, } 1904\end{array}$ \\
\hline Reference & $\begin{array}{l}\text { Present study } \\
\text { (Figs. 4-6) }\end{array}$ & $\begin{array}{c}\text { Stafford } \\
(1904)\end{array}$ & $\begin{array}{c}\text { Manter } \\
\text { (1926, fig. 58) }\end{array}$ & $\begin{array}{l}\text { Linton }(1940, \\
\text { figs. } 278,280)\end{array}$ & $\begin{array}{l}\text { Miller (1941, } \\
\text { fig. 20) }\end{array}$ & $\begin{array}{c}\text { Polyanskii } \\
\text { (1955, fig. 22) }\end{array}$ & $\begin{array}{l}\text { Ronald } \\
(1960)\end{array}$ & $\begin{array}{c}\text { Bray (1979, } \\
\text { 1987, fig. 12A) }\end{array}$ \\
\hline $\mathrm{n}=$ & 1 & 1 & 6 & 2 & 1 & 1 & 4 & 1 \\
\hline Host(s) & $\begin{array}{l}\text { Sebastes } \\
\text { rubrivinctus } \\
\text { (Jordan \& } \\
\text { Gilbert, 1880) } \\
\text { (Sebastidae) }\end{array}$ & $\begin{array}{c}\text { Hippoglossus } \\
\text { hippoglossus } \\
\text { (Linnaeus, 1758) } \\
\text { (Pleuronectidae) }\end{array}$ & $\begin{array}{c}\text { Hippoglossus } \\
\text { hippoglossus } \\
\text { (Linnaeus, 1758) } \\
\text { (Pleuronectidae) }\end{array}$ & $\begin{array}{l}\text { Hippoglossina } \\
\text { oblonga } \\
\text { (Mitchill, 1815) } \\
\text { (Paralichthyidae); } \\
\text { Myoxocephalus } \\
\text { octodecemspinosus } \\
\text { (Mitchill, 1814) } \\
\text { (Cottidae) }\end{array}$ & $\begin{array}{c}\text { Hippoglossus } \\
\text { hippoglossus } \\
\text { (Linnaeus, 1758) } \\
\text { (Pleuronectidae) }\end{array}$ & $\begin{array}{c}\text { Hippoglossus } \\
\text { hippoglossus } \\
\text { (Linnaeus, 1758) } \\
\text { (Pleuronectidae) }\end{array}$ & $\begin{array}{c}\text { Hippoglossus } \\
\text { hippoglossus } \\
\text { (Linnaeus, 1758) } \\
\text { (Pleuronectidae) }\end{array}$ & $\begin{array}{c}\text { Hippoglossus } \\
\text { hippoglossus } \\
\text { (Linnaeus, 1758) } \\
\text { (Pleuronectidae) }\end{array}$ \\
\hline Locality & $\begin{array}{l}\text { Off Newport, } \\
\text { Oregon }\end{array}$ & $\begin{array}{l}\text { Off east coast } \\
\text { of Canada }\end{array}$ & $\begin{array}{l}\text { Off coast of } \\
\text { Maine }\end{array}$ & $\begin{array}{l}\text { Off Woods Hole, } \\
\text { Massachusetts }\end{array}$ & $\begin{array}{l}\text { Off east coast } \\
\text { of Canada }\end{array}$ & Barents Sea & $\begin{array}{l}\text { Off Miscou Bank; } \\
\text { East Point, } \\
\text { Anticosti Island, } \\
\text { Gulf of } \\
\text { St. Lawrence }\end{array}$ & $\begin{array}{c}\text { Off east coast } \\
\text { of Canada }\end{array}$ \\
\hline Length & 1,600 & 2,600 & $\begin{array}{c}3,250 \\
{[\mathrm{n}=1]^{1}}\end{array}$ & $\begin{array}{c}1,750-2,350 \\
(2,050)\end{array}$ & 2,690 & $4,105^{4}$ & $2,500-3,050^{5}$ & $2,772^{4}$ \\
\hline Width at pharynx & 480 & $-^{3}$ & $\begin{array}{c}560 \\
{[n=1]^{1,4}}\end{array}$ & $\begin{array}{c}401 \\
{[n=1]^{1,4}}\end{array}$ & $558^{4}$ & $642^{4}$ & $-^{3}$ & $457^{4}$ \\
\hline Width at VS ${ }^{1}$ & 808 & 810 & $\begin{array}{c}860 \\
{[n=1]^{1}}\end{array}$ & $\begin{array}{c}620-1,000 \\
(810)\end{array}$ & 760 & $1,224^{4}$ & $650-750^{5}$ & $707^{4}$ \\
\hline Width at $\mathrm{T}^{1}$ & 952 & $-^{3}$ & $\begin{array}{c}897 \\
{[n=1]^{1,4}}\end{array}$ & $\begin{array}{l}\sim 700-1,055 \\
\quad(878)^{4}\end{array}$ & $714^{4}$ & $1,030^{4}$ & $-^{3}$ & $652^{4}$ \\
\hline Forebody $\mathrm{L}^{1}$ & 416 & $-^{3}$ & $\begin{array}{c}1,065 \\
{[n=1]^{1,4}}\end{array}$ & $\begin{array}{c}620-927 \\
(774)^{4}\end{array}$ & $893^{4}$ & $1,523^{4}$ & $-^{3}$ & $989^{4}$ \\
\hline Hindbody L & 1,024 & $-^{3}$ & $\begin{array}{c}2,017 \\
{[n=1]^{1,4}}\end{array}$ & $\begin{array}{c}970-1,263 \\
(1,116)^{4}\end{array}$ & $1,607^{4}$ & $2,329^{4}$ & $-^{3}$ & $1,587^{4}$ \\
\hline Oral sucker (OS) L & 120 & $-^{3}$ & $\begin{array}{c}168 \\
{[n=1]^{1,4}}\end{array}$ & $\begin{array}{c}139-144 \\
(142)^{4}\end{array}$ & 200 & $254^{4}$ & $-^{3}$ & $152^{4}$ \\
\hline OS W' ${ }^{1}$ & 180 & $-^{3}$ & $\begin{array}{c}224 \\
{[n=1]^{1}}\end{array}$ & $\begin{array}{c}140-190 \\
(165)\end{array}$ & 150 & $299^{4}$ & $220^{5}$ & $185^{4}$ \\
\hline Prepharynx L & 0 & $-^{3}$ & 0 & $0[\mathrm{n}=1]^{1,4}$ & $0^{4}$ & $0^{4}$ & $-^{3}$ & $0^{4}$ \\
\hline Pharynx L & 52 & $-^{3}$ & $\begin{array}{c}97 \\
{[n=1]^{1}}\end{array}$ & $\begin{array}{l}51 \\
{[n=1]^{1,4}}\end{array}$ & 63 & $134^{4}$ & $-^{3}$ & $76^{4}$ \\
\hline Pharynx W & 44 & $-^{3}$ & $\begin{array}{c}68 \\
{[n=1]^{1}}\end{array}$ & $\begin{array}{c}50-60 \\
(55)\end{array}$ & $100^{4}$ & $134^{4}$ & $-^{3}$ & $54^{4}$ \\
\hline Esophagus L & 244 & $-^{3}$ & $\begin{array}{c}285 \\
{[n=1]^{1}}\end{array}$ & $\begin{array}{c}300-400 \\
(350)\end{array}$ & $234^{4}$ & $358^{4}$ & $-^{3}$ & $348^{4}$ \\
\hline $\begin{array}{l}\text { Intestinal bifurc. } \\
\text { anterior to VS }\end{array}$ & 44 & $-^{3}$ & $\begin{array}{c}462 \\
{[n=1]^{1,4}}\end{array}$ & $\begin{array}{c}240-448 \\
(344)^{4}\end{array}$ & $335^{4}$ & $731^{4}$ & -3 & $413^{4}$ \\
\hline Post-cecal region L & $-^{3}$ & $-^{3}$ & $-^{3}$ & $-^{3}$ & $1,339^{4}$ & $-^{3}$ & $-^{3}$ & $1,228^{4}$ \\
\hline VS L & 136 & $-^{3}$ & $\begin{array}{c}182 \\
{[n=1]^{1,4}}\end{array}$ & $\begin{array}{c}160 \\
(160)^{4}\end{array}$ & $212^{4}$ & $299^{4}$ & $-^{3}$ & $217^{4}$ \\
\hline VS W & 188 & $-^{3}$ & $\begin{array}{c}240 \\
{[n=1]^{1}}\end{array}$ & $\begin{array}{c}140-210 \\
(175)\end{array}$ & 200 & $269^{4}$ & $220^{5}$ & $174^{4}$ \\
\hline $\mathrm{DT}^{1} \mathrm{~L}$ & 192 & $-^{3}$ & $\begin{array}{c}392 \\
{[n=1]^{1,4}}\end{array}$ & $\begin{array}{c}182-240 \\
(211)^{4}\end{array}$ & 370 & $418^{4}$ & $-^{3}$ & $348^{4}$ \\
\hline DT W & 196 & $-^{3}$ & $\begin{array}{c}355 \\
{[n=1]^{1}}\end{array}$ & $\begin{array}{c}190-240 \\
(215)^{4}\end{array}$ & 290 & $388^{4}$ & $-^{3}$ & $261^{4}$ \\
\hline$S T^{1} \mathrm{~L}$ & 160 & $-^{3}$ & $\begin{array}{c}392 \\
{[n=1]^{1,4}}\end{array}$ & $\begin{array}{c}168-224 \\
(196)^{4}\end{array}$ & 350 & $418^{4}$ & $-^{3}$ & $304^{4}$ \\
\hline ST W & 192 & $-^{3}$ & $\begin{array}{c}355 \\
{[n=1]^{1}}\end{array}$ & $\begin{array}{c}153-256 \\
(204)^{4}\end{array}$ & 250 & $358^{4}$ & $-^{3}$ & $228^{4}$ \\
\hline
\end{tabular}


Table 3. Continued.

\begin{tabular}{|c|c|c|c|c|c|c|c|c|}
\hline Parasite & $\begin{array}{l}\text { teganoderma } \\
\text { sp. }\end{array}$ & $\begin{array}{l}\text { S. formosum } \\
\text { Stafford, } 1904\end{array}$ & $\begin{array}{l}\text { S. formosum } \\
\text { Stafford, } 1904\end{array}$ & $\begin{array}{l}\text { S. formosum } \\
\text { Stafford, } 1904\end{array}$ & $\begin{array}{l}\text { S. formosum } \\
\text { Stafford, } 1904\end{array}$ & $\begin{array}{l}\text { S. formosum } \\
\text { Stafford, } 1904\end{array}$ & $\begin{array}{l}\text { S. formosum } \\
\text { Stafford, } 1904\end{array}$ & $\begin{array}{l}\text { S. formosum } \\
\text { Stafford, } 1904\end{array}$ \\
\hline Reference & $\begin{array}{l}\text { Present study } \\
\text { (Figs. 4-6) }\end{array}$ & $\begin{array}{l}\text { Stafford } \\
(1904)\end{array}$ & $\begin{array}{c}\text { Manter } \\
\text { (1926, fig. 58) }\end{array}$ & $\begin{array}{l}\text { Linton }(1940, \\
\text { figs. } 278,280)\end{array}$ & $\begin{array}{l}\text { Miller (1941, } \\
\text { fig. 20) }\end{array}$ & $\begin{array}{c}\text { Polyanskii } \\
(1955, \text { fig. 22) }\end{array}$ & $\begin{array}{l}\text { Ronald } \\
(1960)\end{array}$ & $\begin{array}{c}\text { Bray (1979, } \\
\text { 1987, fig. 12A) }\end{array}$ \\
\hline $\begin{array}{l}\text { Inter-testicular } \\
\text { region (ITR) W }\end{array}$ & 240 & $-^{3}$ & $\begin{array}{c}168 \\
{[n=1]^{1,4}}\end{array}$ & $\begin{array}{c}292-384 \\
(338)^{4}\end{array}$ & $123^{4}$ & $254^{4}$ & $-^{3}$ & $109^{4}$ \\
\hline $\begin{array}{l}\text { Post-testicular } \\
\text { region (PTR) L }\end{array}$ & 688 & $-^{3}$ & $\begin{array}{c}1,107 \\
{[n=1]^{1,4}}\end{array}$ & $\begin{array}{c}569-799 \\
(684)^{4}\end{array}$ & $960^{4}$ & $1,523^{4}$ & $-^{3}$ & $924^{4}$ \\
\hline PTR W at mid-point & nt 680 & $-^{3}$ & $\begin{array}{c}841 \\
{[n=1]^{1,4}}\end{array}$ & $\begin{array}{l}591-879 \\
(735)^{4}\end{array}$ & $636^{4}$ & $761^{4}$ & $-^{3}$ & $554^{4}$ \\
\hline Cirrus pouch (CP) L & L $\quad 504$ & $-^{3}$ & $\begin{array}{c}690 \\
{[n=1]^{1}}\end{array}$ & $\begin{array}{c}450 \\
{[n=1]^{1}}\end{array}$ & $-^{3}$ & $911^{4}$ & $-^{3}$ & $587^{4}$ \\
\hline CP W & 112 & $-^{3}$ & $\begin{array}{c}190 \\
{[n=1]^{1}}\end{array}$ & $\begin{array}{c}102-180 \\
(141)^{4}\end{array}$ & $134^{4}$ & $209^{4}$ & -3 & $130^{4}$ \\
\hline $\begin{array}{l}\text { Seminal vesicle } \\
\text { (SV)_proximal } \\
\text { portion L }\end{array}$ & 80 & $-^{3}$ & $\begin{array}{c}176 \\
{[n=1]^{1}} \\
{[\text { entire SV] }}\end{array}$ & $-^{3}$ & $-^{3}$ & $\begin{array}{c}328^{4} \\
\text { [entire SV] }\end{array}$ & $-^{3}$ & $\begin{array}{c}109^{4} \\
\text { [entire SV] }\end{array}$ \\
\hline $\begin{array}{l}\text { SV-proximal } \\
\text { portion W }\end{array}$ & 68 & $-^{3}$ & $\begin{array}{l}42[\mathrm{n}=1]^{1,4} \\
{[\text { entire SV] }}\end{array}$ & $-^{3}$ & $-^{3}$ & $\begin{array}{c}75^{4} \\
\text { [entire SV] }\end{array}$ & $-^{3}$ & $\begin{array}{c}87^{4} \\
\text { [entire SV] }\end{array}$ \\
\hline SV—distal portion L & L 148 & -3 & & $-^{3}$ & $-^{3}$ & & -3 & \\
\hline SV_-distal portion W & W 132 & $-^{3}$ & & $-^{3}$ & $-^{3}$ & & $-^{3}$ & \\
\hline Pars prostatica (PP) L & ) L 134 & $-^{3}$ & $340[n=1]^{1}$ & $-^{3}$ & $-^{3}$ & $313^{4}$ & $-^{3}$ & $239^{4}$ \\
\hline PP W & 48 & $-^{3}$ & $42[n=1]^{1,4}$ & $-^{3}$ & $-^{3}$ & $60^{4}$ & $-^{3}$ & $33^{4}$ \\
\hline Ejac. duct L & 92 & $-^{3}$ & $170[n=1]^{1}$ & $-^{3}$ & $-^{3}$ & $-^{3}$ & $-^{3}$ & $163^{4}$ \\
\hline Ejac. duct W & 24 & $-^{3}$ & $28[n=1]^{1,4}$ & $-^{3}$ & $-^{3}$ & $-^{3}$ & $-^{3}$ & $33^{4}$ \\
\hline $\begin{array}{l}\text { Genital pore }(\mathrm{GP}) \\
\text { to lateral margin }\end{array}$ & 184 & $-^{3}$ & $\begin{array}{c}126 \\
{[n=1]^{1,4}}\end{array}$ & $\begin{array}{c}96-102 \\
(99)^{4}\end{array}$ & $156^{4}$ & $104^{4}$ & $-^{3}$ & $65^{4}$ \\
\hline GP to anterior end & 400 & $-^{3}$ & $\begin{array}{c}770 \\
{[n=1]^{1,4}}\end{array}$ & $\begin{array}{l}510-607 \\
(559)^{4}\end{array}$ & $614^{4}$ & $1,134^{4}$ & -3 & $772^{4}$ \\
\hline Pre-ovarian region $\mathrm{L}$ & L 608 & $-^{3}$ & $\begin{array}{c}1,247 \\
{[n=1]^{1,4}}\end{array}$ & $\begin{array}{c}729-1,055 \\
(892)^{4}\end{array}$ & $859^{4}$ & $1,373^{4}$ & $-^{3}$ & $1,044^{4}$ \\
\hline Ovary (OV) L & 168 & $-^{3}$ & $\begin{array}{c}210 \\
{[n=1]^{1,4}}\end{array}$ & $\begin{array}{c}160-197 \\
(178)^{4}\end{array}$ & $301^{4}$ & $284^{4}$ & $-^{3}$ & $261^{4}$ \\
\hline OV W & 100 & $-^{3}$ & $\begin{array}{c}260 \\
{[n=1]^{1}}\end{array}$ & $\begin{array}{c}190-272 \\
(231)^{4}\end{array}$ & $-^{3}$ & $358^{4}$ & $-^{3}$ & $261^{4}$ \\
\hline VS to OV & 80 & $-^{3}$ & $\begin{array}{c}14 \\
{[\mathrm{n}=1]^{1,4}}\end{array}$ & $\begin{array}{c}0(0)^{4} \\
\text { [VS contiguous } \\
\text { or overlaps OV] }\end{array}$ & $\begin{array}{c}0^{4} \\
\text { [VS overlaps } \\
\text { OV] }\end{array}$ & $\begin{array}{c}0^{4} \\
\text { [VS and OV } \\
\text { contiguous] }\end{array}$ & $-^{3}$ & $\begin{array}{c}0^{4} \\
\text { [VS overlaps } \\
\text { OV] }\end{array}$ \\
\hline OV to DT & 88 & $-^{3}$ & $\begin{array}{c}350 \\
{[n=1]^{1,4}}\end{array}$ & $\begin{array}{c}175-208 \\
(191)^{4}\end{array}$ & $201^{4}$ & $493^{4}$ & $-^{3}$ & $315^{4}$ \\
\hline $\begin{array}{l}\text { Seminal receptacle } \\
\text { (SR) L }\end{array}$ & $-^{3}$ & $-^{3}$ & $-^{3}$ & $-^{3}$ & $-^{3}$ & $299^{4}$ & $-^{3}$ & $-^{3}$ \\
\hline SR W & $-^{3}$ & $-^{3}$ & $-^{3}$ & $-^{3}$ & $-^{3}$ & $582^{4}$ & $-^{3}$ & $-^{3}$ \\
\hline $\begin{array}{l}\text { No. of Vitelline } \\
\text { follicles (VF)- } \\
\text { dextral field }\end{array}$ & 8 & $10-12$ & $\begin{array}{c}8-9 \\
{[n=1]^{1}}\end{array}$ & $\begin{array}{l}9-13 \\
(11)^{4}\end{array}$ & $8^{4}$ & $8^{4}$ & $-^{3}$ & $9^{4}$ \\
\hline $\begin{array}{l}\text { Dextral vitelline } \\
\text { field L }\end{array}$ & 728 & $-^{3}$ & $\begin{array}{c}672 \\
{[n=1]^{1,4}}\end{array}$ & $\begin{array}{c}306-368 \\
(337)^{4}\end{array}$ & $536^{4}$ & $806^{4}$ & $-^{3}$ & $674^{4}$ \\
\hline $\begin{array}{l}\text { No. of VF- } \\
\text { sinistral field }\end{array}$ & 12 & $10-12$ & $\begin{array}{c}10-12 \\
{[n=1]^{1}}\end{array}$ & $\begin{array}{l}6-16 \\
(11)^{4}\end{array}$ & $9^{4}$ & $10^{4}$ & $-^{3}$ & $10^{4}$ \\
\hline $\begin{array}{l}\text { Sinistral vitelline } \\
\text { field } L\end{array}$ & 328 & $-^{3}$ & $\begin{array}{c}686 \\
{[n=1]^{1,4}}\end{array}$ & $\begin{array}{c}306-448 \\
(377)^{4}\end{array}$ & $547^{4}$ & $821^{4}$ & $-^{3}$ & $641^{4}$ \\
\hline $\begin{array}{l}\text { Dextral field } \\
\text { VF L }\end{array}$ & $\begin{array}{c}108-140 \\
(122) \\
{[n=5]^{1}}\end{array}$ & $-^{3}$ & $\begin{array}{c}112-126 \\
(114) \\
{[n=8]^{1,4}}\end{array}$ & $\begin{array}{c}36-160 \\
(67) \\
{[n=22]^{1,4}}\end{array}$ & $\begin{array}{c}78-123 \\
(100) \\
{[n=8]^{1,4}}\end{array}$ & $\begin{array}{c}134-179 \\
(155) \\
{[n=8]^{1,4}}\end{array}$ & $-^{3}$ & $\begin{array}{c}109-141 \\
(125) \\
{[n=9]^{1,4}}\end{array}$ \\
\hline $\begin{array}{l}\text { Dextral field } \\
\text { VF W }\end{array}$ & $\begin{array}{c}64-108 \\
(86) \\
{[n=5]^{1}}\end{array}$ & $-^{3}$ & $\begin{array}{c}70-140 \\
(102) \\
{[n=8]^{1,4}}\end{array}$ & $\begin{array}{c}29-128 \\
(69) \\
{[n=22]^{1,4}}\end{array}$ & $\begin{array}{c}56-100 \\
(78) \\
{[n=8]^{1,4}}\end{array}$ & $\begin{array}{c}119-164 \\
(147) \\
{[n=8]^{1,4}}\end{array}$ & $-^{3}$ & $\begin{array}{c}98-130 \\
(117) \\
{[n=9]^{1,4}}\end{array}$ \\
\hline
\end{tabular}


Table 3. Continued.

\begin{tabular}{|c|c|c|c|c|c|c|c|c|}
\hline \multirow{2}{*}{$\begin{array}{l}\text { Parasite } \\
\text { Reference }\end{array}$} & \multirow{2}{*}{$\begin{array}{c}\text { Steganoderma } \\
\text { sp. } \\
\text { Present study } \\
\text { (Figs. 4-6) }\end{array}$} & \multirow{2}{*}{$\begin{array}{l}\text { S. formosum } \\
\text { Stafford, } 1904 \\
\text { Stafford } \\
(1904)\end{array}$} & \multirow{2}{*}{$\begin{array}{c}\text { S. formosum } \\
\text { Stafford, } 1904 \\
\text { Manter } \\
(1926, \text { fig. 58) }\end{array}$} & \multirow{2}{*}{$\begin{array}{l}\text { S. formosum } \\
\text { Stafford, } 1904 \\
\text { Linton }(1940, \\
\text { figs. } 278,280)\end{array}$} & \multirow{2}{*}{$\begin{array}{l}\text { S. formosum } \\
\text { Stafford, } 1904 \\
\text { Miller (1941, } \\
\text { fig. 20) }\end{array}$} & \multirow{2}{*}{$\begin{array}{c}\text { S. formosum } \\
\text { Stafford, } 1904 \\
\text { Polyanskii } \\
(1955, \text { fig. 22) }\end{array}$} & \multirow{2}{*}{$\begin{array}{l}\text { S. formosum } \\
\text { Stafford, } 1904 \\
\text { Ronald } \\
(1960)\end{array}$} & \multirow{2}{*}{$\begin{array}{c}\text { S. formosum } \\
\text { Stafford, } 1904 \\
\text { Bray (1979, } \\
\text { 1987, fig. 12A) }\end{array}$} \\
\hline & & & & & & & & \\
\hline $\begin{array}{l}\text { Sinistral field } \\
\text { VF L }\end{array}$ & $\begin{array}{c}120-136 \\
(130) \\
{[n=5]^{1}}\end{array}$ & $-^{3}$ & $\begin{array}{c}84-126 \\
(112) \\
{[n=11]^{1,4}}\end{array}$ & $\begin{array}{c}22-96 \\
(56) \\
{[n=22]^{1,4}}\end{array}$ & $\begin{array}{c}89-134 \\
(107) \\
{[n=9]^{1,4}}\end{array}$ & $\begin{array}{c}119-179 \\
(146) \\
{[n=10]^{1,4}}\end{array}$ & $-^{3}$ & $\begin{array}{c}109-152 \\
(133) \\
{[n=10]^{1,4}}\end{array}$ \\
\hline $\begin{array}{l}\text { Sinistral field } \\
\text { VF W }\end{array}$ & $\begin{array}{c}52-112 \\
(79) \\
{[n=5]^{1}}\end{array}$ & $-^{3}$ & $\begin{array}{c}70-112 \\
(80) \\
{[n=10]^{1,4}}\end{array}$ & $\begin{array}{c}29-160 \\
(78) \\
{[n=22]^{1,4}}\end{array}$ & $\begin{array}{c}78-100 \\
(87) \\
{[n=9]^{1,4}}\end{array}$ & $\begin{array}{c}75-179 \\
(124) \\
{[n=10]^{1,4}}\end{array}$ & $-^{3}$ & $\begin{array}{l}109-163 \\
(122) \\
{[n=10]^{1,4}}\end{array}$ \\
\hline $\begin{array}{l}\text { Pre-vitelline region } \\
\text { (PreVR) L }\end{array}$ & n 392 & $-^{3}$ & $\begin{array}{c}1,079 \\
{[n=1]^{1,4}}\end{array}$ & $\begin{array}{c}656-975 \\
(816)^{4}\end{array}$ & $815^{4}$ & $1,478^{4}$ & $-^{3}$ & $924^{4}$ \\
\hline $\begin{array}{l}\text { Post-vitelline } \\
\text { region (PostVR) L }\end{array}$ & 688 & -3 & $\begin{array}{c}1,485 \\
{[n=1]^{1,4}}\end{array}$ & $\begin{array}{c}795-991 \\
(893)^{4}\end{array}$ & $1,339^{4}$ & $1,881^{4}$ & $-^{3}$ & $1,218^{4}$ \\
\hline Vitelline reservoir L & $\mathrm{L} \quad \mathrm{C}^{3}$ & -3 & $-^{3}$ & $-^{3}$ & -3 & $134^{4}$ & $-^{3}$ & $-^{3}$ \\
\hline Vitelline reservoir $\mathrm{V}$ & $W-3$ & $-^{3}$ & $-^{3}$ & $-^{3}$ & $-^{3}$ & $269^{4}$ & $-^{3}$ & $-^{3}$ \\
\hline Uterus L & 1,344 & $-^{3}$ & $\begin{array}{c}2,325 \\
{[n=1]^{1,4}}\end{array}$ & $\begin{array}{c}1,086-1,679 \\
(1,382)^{4}\end{array}$ & $2,009^{4}$ & $3,284^{4}$ & $-^{3}$ & $2,087^{4}$ \\
\hline $\begin{array}{l}\text { Uterus } W \text { at } \\
\text { widest point } \\
\text { in PTR }\end{array}$ & 768 & $-^{3}$ & $\begin{array}{c}798 \\
{[n=1]^{1,4}}\end{array}$ & $\begin{array}{c}357-863 \\
(610)^{4}\end{array}$ & $592^{4}$ & $925^{4}$ & $-^{3}$ & $522^{4}$ \\
\hline $\begin{array}{l}\text { Post-uterine } \\
\text { region (PUR) L }\end{array}$ & 248 & $-^{3}$ & $\begin{array}{c}280 \\
{[n=1]^{1,4}}\end{array}$ & $\begin{array}{c}64-343 \\
(203)^{4}\end{array}$ & $89^{4}$ & $119^{4}$ & $-^{3}$ & $76^{4}$ \\
\hline Egg L & $\begin{array}{c}28-34(32.0) \\
{[n=5]^{1}}\end{array}$ & $-^{3}$ & $\begin{array}{c}34 \\
{[n=1]^{1}}\end{array}$ & $\begin{array}{c}30-39(34.3) \\
{[n=3]^{1}}\end{array}$ & 36 & $-^{3}$ & $30-39^{5}$ & $-^{3}$ \\
\hline Egg W & $\begin{array}{c}\text { 14-22 (18.8) } \\
{[\mathrm{n}=5]^{1}}\end{array}$ & $-^{3}$ & $\begin{array}{c}17 \\
{[n=1]^{1}}\end{array}$ & $\begin{array}{c}15-24(19.0) \\
{[n=3]^{1}}\end{array}$ & 17 & $-^{3}$ & $12-20^{5}$ & $-^{3}$ \\
\hline Exc. vesicle $L$ & $-^{3}$ & $-^{3}$ & $-^{3}$ & $335[n=1]^{1,4}$ & $-^{3}$ & $-^{3}$ & $-^{3}$ & $-^{3}$ \\
\hline Exc. vesicle W & $-^{3}$ & $-^{3}$ & $-^{3}$ & $96-248(172)^{4}$ & $-^{3}$ & $-^{3}$ & $-^{3}$ & $54^{4}$ \\
\hline Forebody L \%² & 26.0 & $\sim 33.0+$ & $\begin{array}{c}32.8 \\
{[n=1]^{1,4}}\end{array}$ & $\begin{array}{c}35.4-39.4 \\
(37.4)^{4}\end{array}$ & $\sim 40.0$ & $37.1^{4}$ & $-^{3}$ & $35.7^{4}$ \\
\hline OS L \% ${ }^{2}$ & 7.5 & $-^{3}$ & $5.2[n=1]^{1,4}$ & $6.1-7.9(7.0)^{4}$ & 7.4 & $6.2^{4}$ & $-^{3}$ & $5.5^{4}$ \\
\hline Pharynx L \%² & 3.3 & $-^{3}$ & $3.0[n=1]^{1,4}$ & $2.9[n=1]^{1,4}$ & 2.3 & $3.3^{4}$ & $-^{3}$ & $2.7^{4}$ \\
\hline Esophagus L \% ${ }^{2}$ & 15.3 & $-^{3}$ & $8.8[n=1]^{1,4}$ & $\begin{array}{c}17.0-17.1 \\
(17.1)^{4}\end{array}$ & $8.7^{4}$ & $8.7^{4}$ & $-^{3}$ & $12.6^{4}$ \\
\hline VS L $\%^{2}$ & 8.5 & $-^{3}$ & $5.6[n=1]^{1,4}$ & $6.8-9.1(8.0)^{4}$ & $7.9^{4}$ & $7.3^{4}$ & $-^{3}$ & $7.8^{4}$ \\
\hline Width at VS $\%^{2}$ & 50.5 & $-^{3}$ & $26.5[n=1]^{1,4}$ & $\begin{array}{c}35.4-42.6 \\
(39.0)^{4}\end{array}$ & 28.3 & $29.8^{4}$ & $24.6-26.0^{5}$ & $25.5^{4}$ \\
\hline$C P L \%^{2}$ & 31.5 & $-^{3}$ & $21.2[n=1]^{1,4}$ & $19.1[n=1]^{1}$ & $-^{3}$ & $22.2^{4}$ & $-^{3}$ & $21.2^{4}$ \\
\hline$P P L$ as $\%$ of $C P L$ & 26.6 & $-^{3}$ & $49.3[n=1]^{1,4}$ & $-^{3}$ & $-^{3}$ & $34.4^{4}$ & -3 & $40.7^{4}$ \\
\hline $\begin{array}{l}\text { Ejac. duct L } \\
\text { as \% of CP L }\end{array}$ & 18.3 & -3 & $24.6[n=1]^{1,4}$ & $-^{3}$ & $-^{3}$ & $-^{3}$ & $-^{3}$ & $27.8^{4}$ \\
\hline DT L \% ${ }^{2}$ & 12.0 & $-^{3}$ & $12.1[n=1]^{1,4}$ & $\begin{array}{c}10.2-10.4 \\
(10.3)^{4}\end{array}$ & 13.8 & $10.2^{4}$ & $-^{3}$ & $12.6^{4}$ \\
\hline ST L \% ${ }^{2}$ & 10.0 & $-^{3}$ & $12.1[n=1]^{1,4}$ & $9.5-9.6(9.6)^{4}$ & 13.0 & $10.2^{4}$ & $-^{3}$ & $11.0^{4}$ \\
\hline Width at $\mathrm{T} \%^{2}$ & 59.5 & $-^{3}$ & $27.6[n=1]^{1,4}$ & $\begin{array}{l}\sim 40.0-44.9 \\
\quad(42.4)^{4}\end{array}$ & $26.5^{4}$ & $25.1^{4}$ & $-^{3}$ & $23.5^{4}$ \\
\hline OV L \%² & 10.5 & -3 & $6.5[n=1]^{1,4}$ & $6.8-11.3(9.1)^{4}$ & $11.2^{4}$ & $6.9^{4}$ & $-^{3}$ & $9.4^{4}$ \\
\hline VS to OV $\%^{2}$ & 5.0 & $-^{3}$ & $0.4[n=1]^{1,4}$ & $0(0)^{4}$ & $0^{4}$ & $0^{4}$ & $-^{3}$ & $0^{4}$ \\
\hline OV to DT $\%^{2}$ & 5.5 & $-^{3}$ & $10.8[n=1]^{1,4}$ & $8.9-10.0(9.4)^{4}$ & $7.5^{4}$ & $12.0^{4}$ & $-^{3}$ & $11.4^{4}$ \\
\hline $\begin{array}{l}\text { Dextral vitelline } \\
\text { field } L \%^{2}\end{array}$ & 45.5 & $-^{3}$ & $20.7[n=1]^{1,4}$ & $\begin{array}{c}15.7-17.5 \\
(16.6)^{4}\end{array}$ & $19.9^{4}$ & $19.6^{4}$ & $-^{3}$ & $24.3^{4}$ \\
\hline $\begin{array}{l}\text { Sinistral vitelline } \\
\text { field } L \%^{2}\end{array}$ & 20.5 & -3 & $21.1[n=1]^{1,4}$ & $\begin{array}{c}17.5-19.1 \\
(18.3)^{4}\end{array}$ & $20.3^{4}$ & $20.0^{4}$ & -3 & $23.1^{4}$ \\
\hline
\end{tabular}


Table 3. Continued.

\begin{tabular}{|c|c|c|c|c|c|c|c|c|}
\hline Parasite & $\begin{array}{c}\text { Steganoderma } \\
\text { sp. }\end{array}$ & $\begin{array}{l}\text { S. formosum } \\
\text { Stafford, } 1904\end{array}$ & $\begin{array}{l}\text { S. formosum } \\
\text { Stafford, } 1904\end{array}$ & $\begin{array}{l}\text { S. formosum } \\
\text { Stafford, } 1904\end{array}$ & $\begin{array}{l}\text { S. formosum } \\
\text { Stafford, } 1904\end{array}$ & $\begin{array}{l}\text { S. formosum } \\
\text { Stafford, } 1904\end{array}$ & $\begin{array}{l}\text { S. formosum } \\
\text { Stafford, } 1904\end{array}$ & $\begin{array}{l}\text { S. formosum } \\
\text { Stafford, } 1904\end{array}$ \\
\hline Reference & $\begin{array}{l}\text { Present study } \\
\text { (Figs. 4-6) }\end{array}$ & $\begin{array}{l}\text { Stafford } \\
(1904)\end{array}$ & $\begin{array}{c}\text { Manter } \\
\text { (1926, fig. 58) }\end{array}$ & $\begin{array}{l}\text { Linton }(1940, \\
\text { figs. } 278,280)\end{array}$ & $\begin{array}{l}\text { Miller (1941, } \\
\text { fig. 20) }\end{array}$ & $\begin{array}{c}\text { Polyanskii } \\
\text { (1955, fig. 22) }\end{array}$ & $\begin{array}{l}\text { Ronald } \\
(1960)\end{array}$ & $\begin{array}{c}\text { Bray (1979, } \\
\text { 1987, fig. 12A) }\end{array}$ \\
\hline PreVR L \%² & 24.5 & $-^{3}$ & $33.2[n=1]^{1,4}$ & $\begin{array}{c}37.5-41.5 \\
(39.5)^{4}\end{array}$ & $30.3^{4}$ & $36.0^{4}$ & $-^{3}$ & $33.3^{4}$ \\
\hline PostVR L \% ${ }^{2}$ & 43.0 & $-^{3}$ & $45.7[n=1]^{1,4}$ & $\begin{array}{c}42.2-45.4 \\
(43.9)^{4}\end{array}$ & $49.8^{4}$ & $45.8^{4}$ & $-^{3}$ & $43.9^{4}$ \\
\hline PTR L \%² & 43.0 & $-^{3}$ & $34.1[n=1]^{1,4}$ & $\begin{array}{c}32.5-34.0 \\
(33.3)^{4}\end{array}$ & $35.7^{4}$ & $37.1^{4}$ & -3 & $33.3^{4}$ \\
\hline PUR L \% ${ }^{2}$ & 15.5 & $-^{3}$ & $8.6[n=1]^{1,4}$ & $\begin{array}{c}2.7-19.6 \\
(11.2)^{4}\end{array}$ & $3.3^{4}$ & $2.9^{4}$ & $-^{3}$ & $2.7^{4}$ \\
\hline $\begin{array}{l}\text { OS:pharynx } \\
\text { width ratio }\end{array}$ & $1: 4.09$ & $-^{3}$ & $\begin{array}{c}1: 3.29 \\
{[n=1]^{1,4}}\end{array}$ & $\begin{array}{c}1: 2.80-3.17 \\
(1: 2.99)\end{array}$ & $1: 1.50^{4}$ & $1: 2.23^{4}$ & $-^{3}$ & $1: 3.43^{4}$ \\
\hline Sucker $L$ ratio & $1: 1.13$ & $-^{3}$ & $\begin{array}{c}1: 1.08 \\
{[n=1]^{1,4}}\end{array}$ & $\begin{array}{c}1: 1.11-1.15 \\
(1: 1.13)^{4}\end{array}$ & $1: 1.06^{4}$ & $1: 1.18^{4}$ & $-^{3}$ & $1: 1.43^{4}$ \\
\hline Sucker $\mathrm{W}$ ratio & $1: 1.04$ & $-^{3}$ & $\begin{array}{c}1: 1.07 \\
{[n=1]^{1,4}}\end{array}$ & $\begin{array}{c}1: 1.00-1.11 \\
(1.06)\end{array}$ & $1: 1.33$ & $1: 0.90^{4}$ & $1: 1.00^{5}$ & $1: 0.94^{4}$ \\
\hline
\end{tabular}

1 DT, dextral (right) testis; L, length; ST, sinistral (left) testis; T, testes; VS, ventral sucker; W, width; ranges followed by mean in parentheses where applicable; number [n] of measurements provided if different from total number of worms examined.

2 Proportion of body length.

3 -, this feature was either not observed, not measured, not described, and/or not illustrated.

4 This measurement was either not available or was in error in the original publication and calculated from original illustration(s).

5 Ronald (1960, p. 931) provided measurements and size ranges for some features of S. formosum; however, raw data was not provided from which to calculate averages.

ensis are smaller than S. eamiqtrema, while S. formosum, $S$. oviformis, and S. rhiphidium are larger. Our specimens of $S$. eamiqtrema have an elongate oval to spindle-shaped body and differ in overall size $(1,140-1,840 \times 584-720$; Fig. 1) from congeners; whereas, $S$. atherinae is "spearheadshaped" (1,220-1,360 × 425-544; see Price, 1934, fig. 3), S. formosum is elongate oval $(2,600-2,772 \times 707-810$; see Stafford, 1904; Bray, 1987, fig. 12A), S. rhiphidium is noticeably pyriform $(2,560 \times 1,260$; see Wang, 1986, fig. 6$)$, $S$. macrophallus is lanceolate $(700-800 \times 280$; see Szidat and Nani, 1951, fig. 5), S. oviformis is broadly oval, almost circu$\operatorname{lar}(1,900 \times 1,600$ and 2,300 $\times 1,480$; see Szidat, 1962, fig. $1)$, S. szidati is oval (822-1,870 $\times 397-963$; see Viozzi et al., 2000, fig. 1a) and S. valchetensis is oval (400-645 × 168392; see Etchegoin et al., 2002, fig. 1). The forebody length of S. szidati is described by Viozzi et al. (2000) as occupying $49 \%$ of the body length yet their fig. 1a illustrates a forebody length about $29 \%$ of body length, which is more in line with our specimens of S. eamiqtrema (29.7\%-37.8\% of body length). Our specimens of $S$. eamiqtrema have a larger ventral than oral sucker (sucker length ratio $=$ $1: 0.92-1.97$; sucker width ratio $=1: 1.00-1.69$ ), but the oral sucker is larger in $S$. atherinae (sucker length ratio $=1: 0.53$ 0.63 ; sucker width ratio $=1: 0.90-1.08$ ) while the ventral sucker is considerably larger and more robust in S. ovifor- mis (sucker length ratio $=1: 2.55-2.83$; sucker width ratio $=$ 1:3.05-3.28). The oral and ventral sucker lengths as a proportion of body length in our material is $7.9 \%-12.6 \%$ and 10.6\%-20.7\% (see Table 2); however, it is noticeably larger in $S$. valchetensis (oral sucker $=22.5 \%-22.8 \%$; ventral sucker $=22.5 \%-30.9 \%$, respectively; see Etchegoin et al., 2002, fig. 1) and in S. oviformis (ventral sucker $=28.3 \%$ 28.9\%). A prepharynx is absent in our specimens; however, this feature is described by Price (1934) as 20-40 $\mu \mathrm{m}$ long in $S$. atherinae, and it might be present in S. macrophallus (see Szidat and Nani, 1951, fig. 5a). Pharynx length as a proportion of body length was either larger $(6.5 \%-6.6 \%$ in S. atherinae, $5.6 \%$ in S. rhiphidium, $7.5 \%-8.6 \%$ in S. macrophallus, $8.3 \%-10.1 \%$ in S. valchetensis) or smaller (2.7\% in S. formosum) than in S. eamiqtrema (3.8\%-5.1\%), and the esophagus length shorter (or even absent!) in other species of Steganoderma (i.e., very short or absent in S. atherinae, $2.4 \%$ of body length in S. szidati, $1.5 \%$ in S. valchetensis vs. $7.4 \%-16.1 \%$ in the new species). Manter (1926, p. 89) described the esophagus of $S$. formosum as splitting into "two short branches" so that the intestine per se did not start at the bifurcation, as the esophagus extended histologically beyond that point; we saw no evidence of these "two short branches" in our specimens of S. eamiqtrema. Oral sucker to pharynx width ratio in S. eamiqtrema mea- 
sured 1:2.26-2.67 (i.e., oral sucker about $2 \frac{1}{2} 2$ times wider than pharynx); this ratio differed in S. atherinae (1:1.672.22), S. formosum (1:3.43), S. rhiphidium (1:1.71), S. oviformis (1:1.67-2.00) and in S. valchetensis (1:2.50-3.69). As similarly described for $S$. formosum (discussed later), $S$. eamiqtrema possessed granular parenchymal cells around the pharynx and esophagus (we could not confirm their presence in smaller specimens-we observed them only in our two largest specimens); these cells were neither described nor illustrated for the six other recognized species in Steganoderma. The ceca may extend either posterior to the testes (S. atherinae, S. szidati) or only to the pre-testicular region between the ventral sucker and the ovary/anterior margin of the vitelline fields (S. rhiphidium) compared to extending to or near the level of the testes in the new species [Note: Viozzi et al., 2000, p. 205 described the ceca of S. szidati as "reaching to level of posterior border of testes or beyond"; however, in fig. 1a the ceca of this species reach only to the mid-level of the testes], and the ceca of $S$. formosum appear noticeably more thick-walled than in S. eamiqtrema (see Bray, 1987, fig. 12A). The testes are either lobed (S. atherinae, S. rhiphidium, S. macrophal(us), or as in S. oviformis, irregular in shape, usually almost square, and with three or four deep incisions (cf., lobes) (see Szidat, 1962, fig. 1); they are smooth to slightly indented and globular to oval in our specimens of S. eamiqtrema. The cirrus pouch is distinctly pyriform in S. atherinae, completely pre-acetabular in S. rhiphidium and in $S$. oviformis, extends only to the anterior margin of the ventral sucker in S. valchetensis, or is noticeably large in proportion to body size as in S. macrophallus (see Szidat and Nani, 1951, fig. 5b); in our material the cirrus pouch is clavate to comma-shaped and runs to a level at or near the posterior margin of the ventral sucker. The seminal vesicle is "somewhat twisted" in S. atherinae (see Price, 1934, p. 4) and appears unipartite and saccate in S. formosum, $S$. rhiphidium, and S. macrophallus (see Szidat and Nani, 1951, fig. 5b; Wang, 1986, fig. 6; Bray, 1987, fig. 12A), while in our specimens the seminal vesicle is bipartite with the proximal portion large and saccate to oblong oval in shape and the distal portion smaller, saccate, and round [Note: Szidat, 1962, p. 69 mentioned for S. oviformis that the seminal vesicle was surrounded by numerous "túbulos glandulares" or glandular tubules (cf. prostatic cells) but did not mention whether the seminal vesicle was unipartite or bipartite. The worm illustrated on the left in fig. 1 of Szidat, 1962, possesses a bipartite seminal vesicle, yet this feature in the worm illustrated on the right of this figure (and redrawn for the species key that follows) appears to be unipartite]. The location of the genital pore in $S$. eamiqtrema is submedian (sinistral) at the level of the in- testinal bifurcation or just posterior to it; however, it is noticeably post-bifurcal in S. atherinae (see Price, 1934, fig. 3), pre-bifurcal "between the pharynx and the edge of the body" in S. macrophallus as described by Szidat and Nani (1951, p. 347) [Note: their fig. 5 illustrates the genital pore at the level of the esophagus], pre-bifurcal and "approximately at the height of the pharynx" in S. oviformis as described by Szidat (1962, p. 69) [Note: fig. 1 of this species illustrates this feature at the level of the oral sucker], and pre-bifurcal at the level of the pharynx in both S. valchetensis and S. szidati (see Viozzi et al., 2000, fig. 1a; Etchegoin et al., 2002, fig. 1) as well as marginal in the latter species. The ovary is lobed in S. atherinae, S. rhiphidium, and S. oviformis, and it is described and illustrated as subtriangular and bean-shaped in S. formosum (see Linton, 1940, fig. 280); it is smooth and globular to oval to subcircular in our material. Szidat and Nani (1951) described S. macrophallus as lacking a seminal receptacle (see Bray, 1987, pp. 108-110 for a discussion of this feature in S. formosum), while it is present, though often inconspicuous, in our specimens of S. eamigtrema. Compared with the new species, there is a noticeable distance between the ventral sucker and ovary in S. rhiphidium and between the ovary and dextral testis in S. atherinae, S. formosum, and S. rhiphidium while there is either a much shorter distance (S. valchetensis) or the ovary overlaps the dextral testis (S. szidati). We noticed considerable variability in the number, shape, and/or position of the vitellaria between our specimens of S. eamiqtrema and other species of Steganoderma. The vitellaria of the new species are distributed in two symmetrical fields that extend posteriorly within the anterior hindbody from the mid-level of the cirrus pouch (i.e., at about the level of the anterior margin of the ventral sucker) to the level of either the ovary or the anterior half of testes; the follicles are large and relatively few in number (8-10 in the dextral/aporal field, 12 in the sinistral/poral field). The vitelline fields in S. atherinae extend from the posterior margin of the ventral sucker to the anterior margins of the testes, and the follicles themselves are considerably smaller and more numerous (see Price, 1934, fig. 3). While the overall appearance and distribution of the vitelline follicles in S. formosum is similar to the new species, the number of follicles varies somewhat (912 in the dextral/aporal field, 10-12 in the sinistral/poral field; see Stafford, 1904; Bray, 1987, fig. 12A). In S. rhiphidium, the paired vitelline fields are composed of 10 follicles each and have a limited longitudinal and post-acetabular distribution from the posterior ends of the ceca to the anterior margins of the testes (see Wang, 1986, fig. 6). The number of follicles and overall appearance of the vitelline fields in S. macrophallus and S. oviformis are similar 
to our specimens of $S$. eamiqtrema except that the fields appear to extend into the posterior forebody in the former two species (see Szidat and Nani, 1951, fig. 5a; Szidat, 1962, fig. 1). The vitelline fields in S. szidati overlap in follicle number (6-11 in the dextral/aporal field, 9-13 in the sinistral/poral field) and are distributed from the level of the anterior margin of the ventral sucker to the posterior margin of the testes. While the vitelline fields in S. valchetensis also overlap in follicle number (7-13 in the dextral/ aporal field, 8-13 in the sinistral/poral field) with our specimens, their distribution is markedly more anterior extending from the intestinal bifurcation in the forebody to about the posterior half of the ventral sucker and anterior to both the ovary and testes. Vitelline follicle size is also noticeably smaller in S. atherinae (15-31 × 10-15), S. macrophallus (14-23 × 14-18), and S. valchetensis $(19-35 \times 15-$ 27) vs. S. eamiqtrema $(68-100 \times 48-80)$. In the illustration of S. formosum (Syn. Steganoderma messjatzevi [Issaitschikov, 1928] Yamaguti, 1934) by Polyanskii (1955, fig. 22), the vitelline reservoir is oval, conspicuous $(134 \times 269$ - see Table 3) and immediately posterior to the ovary and dextral to the ventral sucker; however, this feature in S. eamiqtrema was quite small $(34-80 \times 20-72)$, saccate to elongate, and either overlapped dorsally by the left margin of the ovary or located immediately sinistral to it. The uterine loops do not appear to extend as close to the posterior extremity in S. macrophallus and in S. szidati as they do in our material, and the metraterm is well-developed with thick walls and surrounded by gland cells in S. oviformis (see Szidat, 1962, fig. 1) while this same feature is noticeably muscular in S. szidati (see Viozzi et al., 2000, fig. 1b); the metraterm was inconspicuous in our specimens of $S$. eamiqtrema (i.e., light stain). Egg size in our specimens (30-36 × 18-26) overlapped that of S. atherinae $(32 \times 18)$, S. formosum (30-39 × 12-24; see Table 3), S. macrophallus (34 × 18-20), S. szidati (26-36 × 14-24), and S. valchetensis (28-34 × 15-19); however, egg size was somewhat larger in S. rhiphidium $(45-54 \times 21-24)$ and in S. oviformis $(40 \times 15)$. The excretory vesicle in the new species is Ishaped, narrow at the posterior end and noticeably expands in width after a short distance anterior; we observed it to extend at least to the level of the ovary. The excretory vesicle of S. rhiphidium is entirely narrow and extends anteriorly to the level of the testes, which are post-ovarian (see Wang, 1986, fig. 6); in S. formosum it was described by Manter (1926, p. 89, figs. 59, 60) as extending from the posterior extremity almost to the posterior margin of the ventral sucker "where it spreads out laterally, T-like"; it is very large and of a "rounded shape" in S. macrophallus extending to about midway within the post-testicular region (see Szidat and Nani, 1951, p. 347 and fig. 5); it appears to expand in width a short distance anterior from the excretory pore in S. szidati (see Viozzi et al., 2000, fig. 1a) and reaches the gonads in immature specimens (fig. 2); and it appears saccate with thick walls in S. valchetensis, reaching only midway within the post-testicular region (see Etchegoin et al., 2002, fig. 1).

\section{Steganoderma sp.}

(Figs. 4-6)

Description: Measurements based on 1 adult, wholemounted, lightly stained, damaged specimen with tear from lower right quadrant across worm at angle to upper left quadrant. Measurements, ratios, and proportions given in Table 3. With the characteristics of the genus. Body oval, widest in middle third of body. Forebody concave due to damage with broadly rounded anterior extremity; hindbody markedly attenuated in posterior third of body, posterior extremity invaginated. Tegument spined; spines larger and denser in anterior half of body, not observed in posterior half of body. Pre-oral lobe absent. Oral sucker oval, transversely elongate, subterminal, wider than long. Ventral sucker oval, transversely elongate, median, slightly larger than oral sucker, at junction of first and second quarter of body. Prepharynx not observed (Note: forebody contracted so pharynx and oral sucker are overlapped). Pharynx small, round. Esophagus straight, distinct, moderately long. Intestinal bifurcation in forebody, pre-acetabular and closer to ventral sucker than anterior extremity. Ceca narrow, dark-stained, bowed at midpoint of length, anterior portion distinct, posterior portion indistinct, extend posteriorly to near level of testes, arcuate posteriorly and terminate blindly; post-cecal distance

Figures 4-6. Steganoderma sp. (Digenea: Zoogonidae: Lepidophyllinae) from the intestine of the flag rockfish, Sebastes rubrivinctus (Jordan and Gilbert, 1880) (Scorpaeniformes: Sebastidae). 4. Adult, ventral view. 5. Male terminal genitalia, ventral view. 6. Posterior extremity showing excretory system, ventral view. Note: This specimen was damaged with a tear from lower right quadrant across worm at angle to upper left quadrant. Abbreviations: C, Cecum; CP, Cirrus pouch; DSC, Dark-staining cells; DSV, Distal seminal vesicle; E, Esophagus; ED, Ejaculatory duct; EP, Excretory pore; EV, Excretory vesicle; GP, Genital pore; OS, Oral sucker; OV, Ovary; P, Pharynx; PC, Prostatic cells; PP, Pars prostatica; PSV, Proximal seminal vesicle; S, Spines; SD, Small duct; T, Tes-

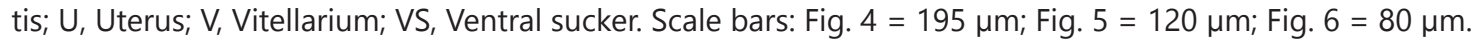




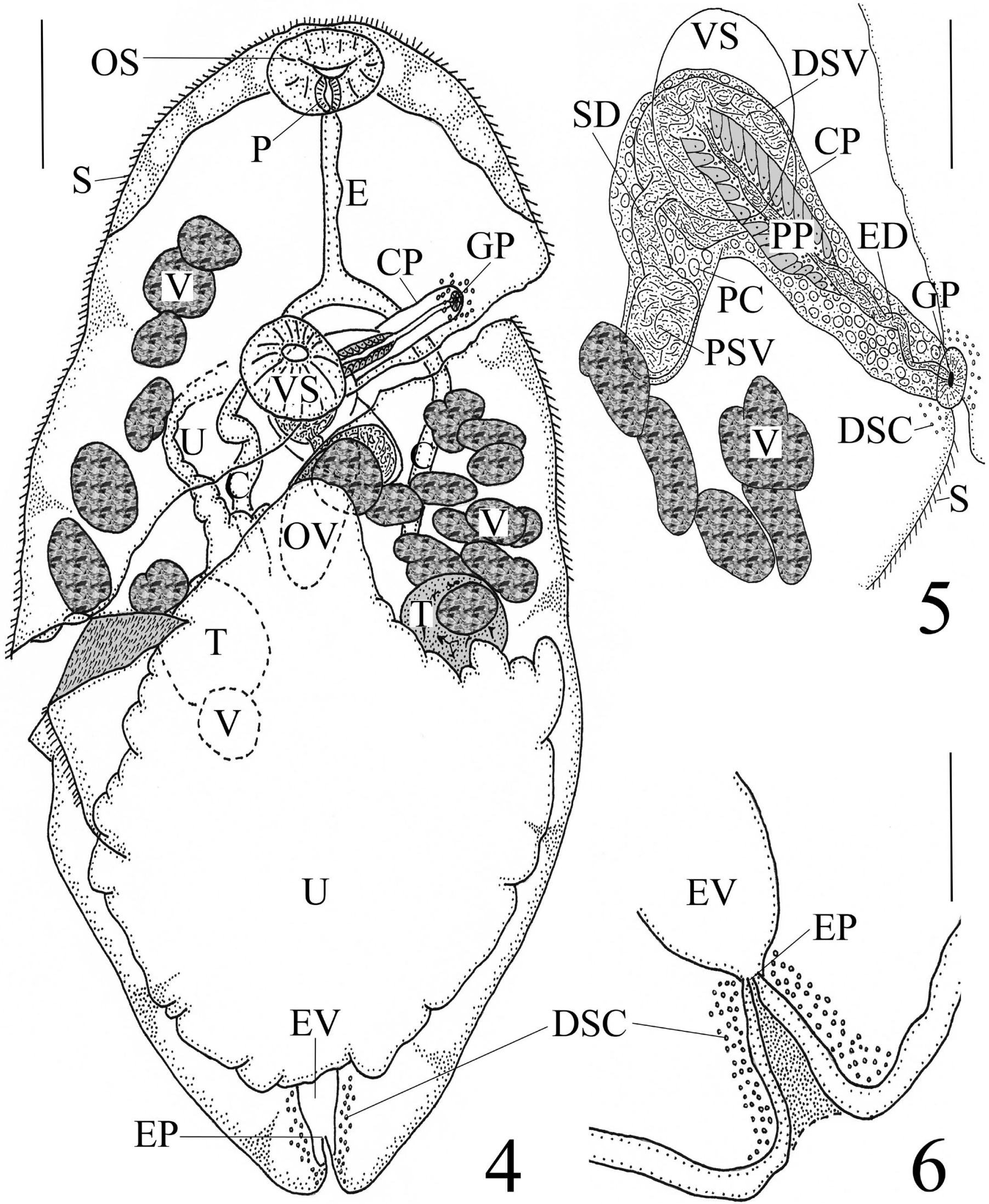


not observed due to damage.

Testes 2, small, dark-stained, very slightly indented, round to globular, opposite, in middle third of body, ventrally overlapped by vitelline fields. Cirrus pouch large, distinct, clavate to comma shaped, extends posterio-dextrally along transverse course from genital pore to midline of worm where it dorsally overlaps ventral sucker before it curves back posterio-sinistrally to run to near mid-level of left field of vitelline follicles. Seminal vesicle bipartite; proximal portion small, round; distal portion larger than proximal portion, saccate; both portions connected by small duct, $80 \times 50$. Prostatic cells indistinct, denser numbers in proximal portion of cirrus pouch and around small duct that connects both portions of seminal vesicle. Pars prostatica tubular, conspicuous, occupies $1 / 4$ of cirrus pouch length and filled with bleb-like cells; ejaculatory duct tubular, widest at distal end, occupies almost $1 / 5$ of cirrus pouch length; cirrus present. Genital atrium deep, round, surrounded by dense number of dark-staining cells. Genital pore submedian, sinistral, close to left margin, and at level of intestinal bifurcation.

Ovary small, dark-stained, elongate oval and longitudinally extended due to damage, mostly smooth but indented at posterior margin, submedian and just sinistral to midline, pre-testicular, posterio-sinistral and close to ventral sucker with anterior margin dorsally overlapped by proximal portion of cirrus pouch; distance of ovary to left testis, 128 or $8.0 \%$ of body length. Proximal female system difficult to delineate due to damage; seminal receptacle not observed but expected to be present and canalicular; Laurer's canal not observed but expected to be present with dorsal opening; Mehlis' gland and vitelline reservoir not observed but expected to be near ovary. Uterus extensive, conspicuous, coiled with many loops, confined almost exclusively to hindbody and occupies most of post-testicular region, extends almost to posterior extremity, proceeds anteriorly through intertesticular region and ventrally overlaps ovary and right testis as well as medial and posterio-sinistral margins of left testes before loops pass farther anterior and to right of cirrus pouch; path of distal uterine loops disrupted due to damage but expected to proceed sinistrally to genital pore. Metraterm not observed due to damage but expected to be present. Vitellaria in two conspicuous symmetrical fields of follicles along lateral margins; right field of follicles distorted by damage and extend posteriorly from level of esophagus to just posterior to right testis; left field of follicles extend posteriorly from level of ventral sucker to left testis; follicles large, oblong to oval to globular to irregular in shape. Eggs numerous, small, either collapsed or crenulated, operculate, clear to amber, non-filamented, non-embryonated.

Excretory vesicle I-shaped, moderately wide, anterior extent not observed due to occlusion by uterus and eggs; dense number of dark-staining cells surround posterior extent of vesicle. Excretory pore terminal and proximate to invaginated posterior extremity of worm.

Host: Sebastes rubrivinctus (Jordan and Gilbert, 1880) (Syns. Sebastichthys rubrivinctus Jordan and Gilbert, 1880; Sebastodes rubrivinctus [Jordan and Gilbert, 1880]) (Scorpaeniformes: Sebastidae); flag rockfish.

Locality: Northeastern Pacific Ocean, approx. $13 \mathrm{~km}$ off Newport, Oregon, $44^{\circ} 38^{\prime} 20.2^{\prime \prime} \mathrm{N}, 124^{\circ} 13^{\prime} 37.4^{\prime \prime} \mathrm{W}$; depth = $200 \mathrm{~m}$.

Site of infection: Intestine.

Deposited material: HWML 42863 (1 slide).

Remarks: While this particular specimen was damaged and only lightly stained, we were able to assign it to the Lepidophyllinae within the Zoogonidae as well as to the genus Steganoderma based, in part, on its possession of the same diagnostic combinations of subfamily and generic morphological characters listed earlier and used to identify our specimens of S. eamiqtrema.

With only one individual of Steganoderma from Se. rubrivinctus, it is impossible to observe any intra-specific variation and any detailed comparative analyses of this specimen with either the currently recognized species of Steganoderma or our specimens of S. eamiqtrema from the present study would be of limited value. This specimen is also lightly stained (i.e., limited contrast of many features) and damaged (it has a tear from the lower right quadrant across the worm at an angle to the upper left quadrant-see Fig. 4). Resultantly, we have elected to designate this digenean from Se. rubrivinctus simply as Steganoderma sp.

Future parasitological studies of species of Sebastes Cuvier, 1829, particularly Se. rubrivinctus, from the deeper waters off Oregon and northern California are recommended in hopes of obtaining additional specimens of this species of Steganoderma. This is so that any intraspecific variability can be observed, a complete species identification can be obtained, and/or a new species can be documentedpotentially, the third species of Steganoderma known from the deep sea.

Stafford (1904) erected Steganoderma with the description of $S$. formosum. At times this genus contained two subgenera distinguishable by the length of the ceca and 
the nature of the ventral sucker: Steganoderma (Steganoderma) Stafford, 1904 contained species with ceca that extended to the testes or not as far and that possessed a sessile ventral sucker; and Steganoderma (Lecithostaphylus) Odhner, 1911 contained species with ceca that extended posterior to the testes and that possessed a pedunculate ventral sucker (Yamaguti, 1958; Bray, 1987). Currently, neither subgenus is recognized, and Steganoderma and Lecithostaphylus Odhner, 1911 are each valid taxa of generic rank (Yamaguti, 1971; Bray, 2008b; WoRMS, 2020a, 2020b). The number of accepted species of Steganoderma also has varied over time (e.g., ten spp. [Yamaguti, 1958], four spp. [Yamaguti, 1971], nine spp. [Bray, 1987]); currently eight species are recognized (WoRMS, 2020b; Blend et al., 2020; Present study). One of the latest keys to the species of Steganoderma is Bray (1987, p. 107-108); however, of the nine species of Steganoderma recognized in that paper, only four are considered still in this genus.

As there has been considerable taxonomic change within Steganoderma since the last comprehensive work(s)/key(s) to this genus, we now present an updated key to the eight species we recognize in this genus.

\section{Key to the Species of Steganoderma Stafford, 1904}

1a. Cirrus pouch limited to forebody (extends as far as anterior margin of ventral sucker) ... 2

1b. Cirrus pouch in forebody and hindbody (extends to mid-level of ventral sucker or further posterior) . . . 4

2a. Genital pore bifurcal . . Steganoderma rhiphidium Wang, 1986 (see Fig. 7)
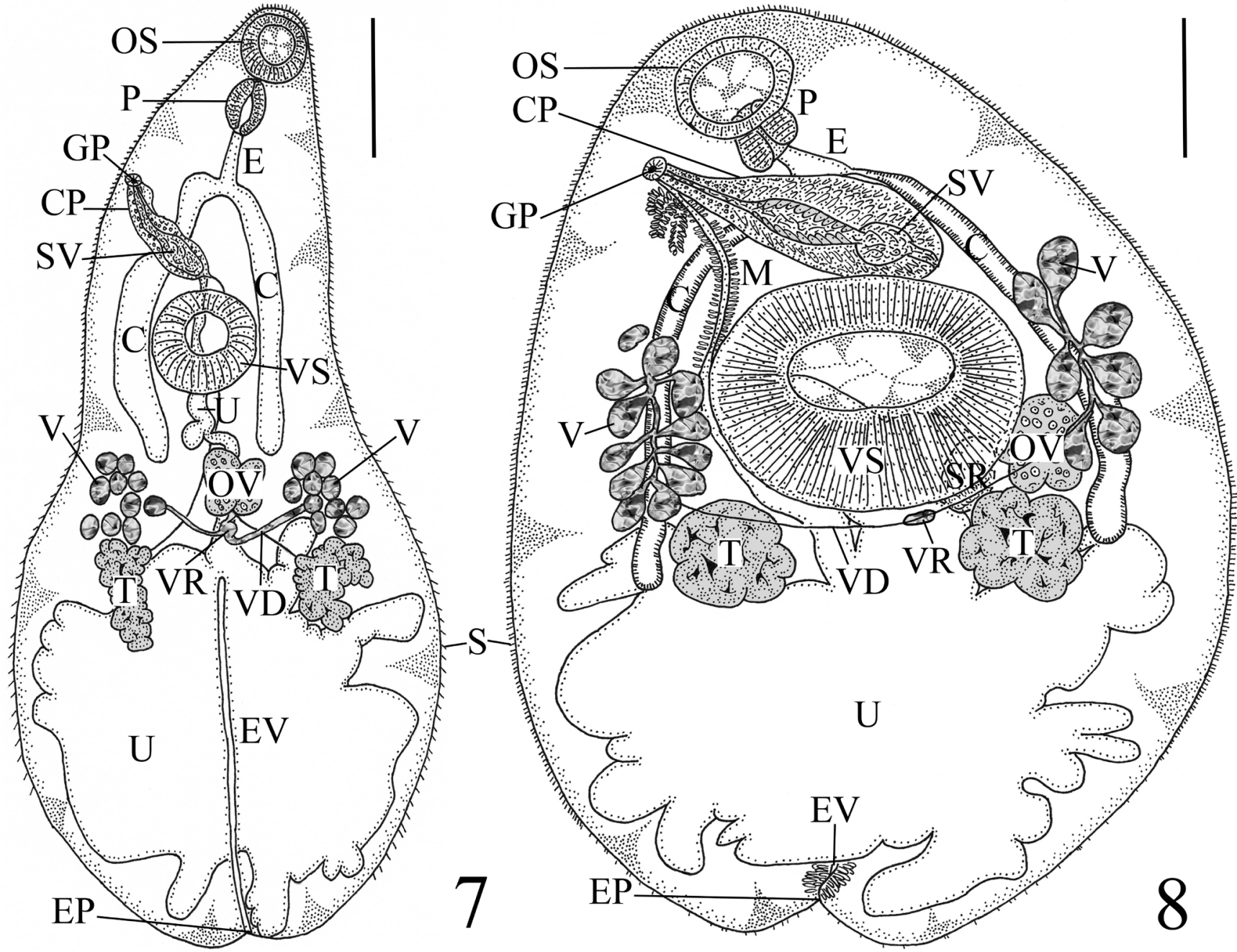

Figures 7-8. Steganoderma rhiphidium Wang, 1986 and Steganoderma oviformis Szidat, 1962 (Digenea: Zoogonidae: Lepidophyllinae). 7. S. rhiphidium, dorsal view, redrawn from Wang (1986, fig. 6). 8. S. oviformis, dorsal view, redrawn from Szidat (1962, fig. 1). Abbreviations: C, Cecum; CP, Cirrus pouch; E, Esophagus; EP, Excretory pore; EV, Excretory vesicle; GP, Genital pore; M, Metraterm; OS, Oral sucker; OV, Ovary; P, Pharynx; S, Spines; SR, Seminal receptacle; SV, Seminal vesicle; T, Testis; U, Uterus; V, Vitellarium; VD, Vitelline duct; VR, vitelline reservoir; VS, Ventral sucker. Scale bars: Fig. $7=400 \mu \mathrm{m}$; Fig. $8=325 \mu \mathrm{m}$. 
2b. Genital pore pre-bifurcal ... 3

3a. Sucker width ratio $\geq 1: 3.00$; testes and ovary noticeably lobed (i.e., 3-5 lobes); body large, broadly oval and almost circular . . Steganoderma oviformis Szidat, 1962 (Syn. Lepidophyllum oviformis [Szidat, 1962] Brooks and McLennan, 1993) (see Fig. 8)

3b. Sucker width ratio 1:1.00-1.65; testes and ovary oval and smooth; body small, oval ... Steganoderma valchetensis Etchegoin, Cremonte and Escalante, 2002 (see Fig. 9)

4a. Genital pore pre-bifurcal ... 5

4b. Genital pore bifurcal or post-bifurcal ... 6

5a. Seminal vesicle unipartite, large, saccate; testes irregular, lobed; vitelline fields can extend into forebody . . Steganoderma macrophallus Szidat and Nani, 1951 (Syns. Lepidophyllum macrophallos [Szidat and Nani, 1951] Brooks and McLennan, 1993; Limnoderetrema macrophallus [Szidat and Nani, 1951] Torres and Neira, 1991) (see Fig. 10)

5b. Seminal vesicle bipartite, each portion round to elongate oval; testes oval, smooth; vitelline fields restricted to hindbody .. . Steganoderma szidati Viozzi, Flores and Ostrowski de Núñez, 2000 (see Fig. 11)

6a. Body "spearhead shaped"; oral sucker larger than ventral sucker; cirrus pouch distinctly pyriform; testes and ovary noticeably lobed . . . Steganoderma atherinae (Price, 1934) Manter, 1947 (Syns. Lecithostaphylus atherinae Price, 1934; Lepidophyllum atherinae [Price, 1934] Brooks and McLennan, 1993) (see Fig. 12)

$6 \mathrm{~b}$. Body elongate oval to spindle-shaped; ventral sucker either larger than oral sucker or equal in size; cirrus pouch clavate to comma-shaped, at times almost retroflexed; testes and ovary smooth ... 7

7a. Body larger, 2,600-2,772 × 707-810; seminal vesicle unipartite, relatively poorly developed; relatively large distance between ovary and testes . . . Steganoderma formosum Stafford, 1904 [type species of genus] (Syns. Deretrema messjatzevi [Issaitschikov, 1928] Manter,
1954; Nordosstrema messjatzevi Issaitschikov, 1928; Steganoderma messjatzevi [Issaitschikov, 1928] Yamaguti, 1934) (see Fig. 13)

7b. Body smaller, 1,140-1,840 × 584-720; seminal vesicle bipartite, each portion distinctly saccate; relatively small distance between ovary and testes ... Steganoderma eamiqtrema n. sp. (see Figs. 1-3)

\section{Discussion}

Earlier, we discussed the list of parasites reported from Se. elongatus and Se. rubrivinctus (Table 1) and touched upon their taxonomic diversity. Relevant to this is the zoogonid Deretrema cholaeum McFarlane, 1936 (Lepidophyllinae); $D$. cholaeum was originally described by McFarlane (1936) from the gall bladder of Sebastes sp. (Syn. Sebastodes sp.)an unidentified species of rockfish (termed a "rock cod") found in Departure Bay, British Columbia. Subsequently, D. cholaeum (Syn. Deretrema pooli Annereaux, 1947) has been reported infecting the bile duct, gall bladder, and intestine of unidentified species of Sebastes in the NE Pacific Ocean from off British Columbia and Santa Cruz, California (Annereaux, 1947; Yamaguti, 1958, 1971; Pratt and McCauley, 1961; Chapa, 1969, 1976; Love and Moser, 1976, 1983; Margolis and Arthur, 1979; Gibson, 1996). Deretrema cholaeum has been documented from one of the host species in this study, Se. elongatus, also collected from the NE Pacific Ocean off British Columbia (Sekerak, 1975; Love and Moser, 1976, 1983; Sekerak and Arai, 1977; Margolis and Arthur, 1979; Gibson, 1996; Love et al., 2002; see our Table 1). While both lepidophyllines, members of Deretrema Linton, 1910 differ from those of Steganoderma in that the vitellarium extends into or is confined to the forebody, the genital pore is lateral (i.e., marginal) and in the mid to posterior forebody, and the excretory vesicle is comparatively longer in the former, while the vitellarium is only in the hindbody, the genital pore is sublateral (i.e., submedian, sinistral) and in the mid to anterior forebody, and the excretory vesicle is comparatively shorter in the latter (see Bray, 2008b).

The finding of S. eamiqtrema in Se. elongatus represents the second species of zoogonid known from this host. The finding of an unidentified species of Steganoderma in Se.

Figures 9-10. Steganoderma valchetensis Etchegoin, Cremonte and Escalante, 2002 and Steganoderma macrophallus Szidat and Nani, 1951 (Digenea: Zoogonidae: Lepidophyllinae). 9. S. valchetensis, ventral view, redrawn from Etchegoin et al. (2002, fig. 1). 10. S. macrophallus, ventral view, redrawn from Szidat and Nani (1951, fig. 5a). Abbreviations: $C_{1}, C e c u m ; C P, C i r r u s$ pouch; $E_{1}$ Esophagus; EP, Excretory pore; EV, Excretory vesicle; GP, Genital pore; M, Metraterm; OS, Oral sucker; OV, Ovary; P, Pharynx; S, Spines; SR, Seminal receptacle; SV, Seminal vesicle; T, Testis; U, Uterus; V, Vitellarium; VS, Ventral sucker. Scale bars: Fig. $9=60$ mm; Fig. $10=95 \mu \mathrm{m}$. 

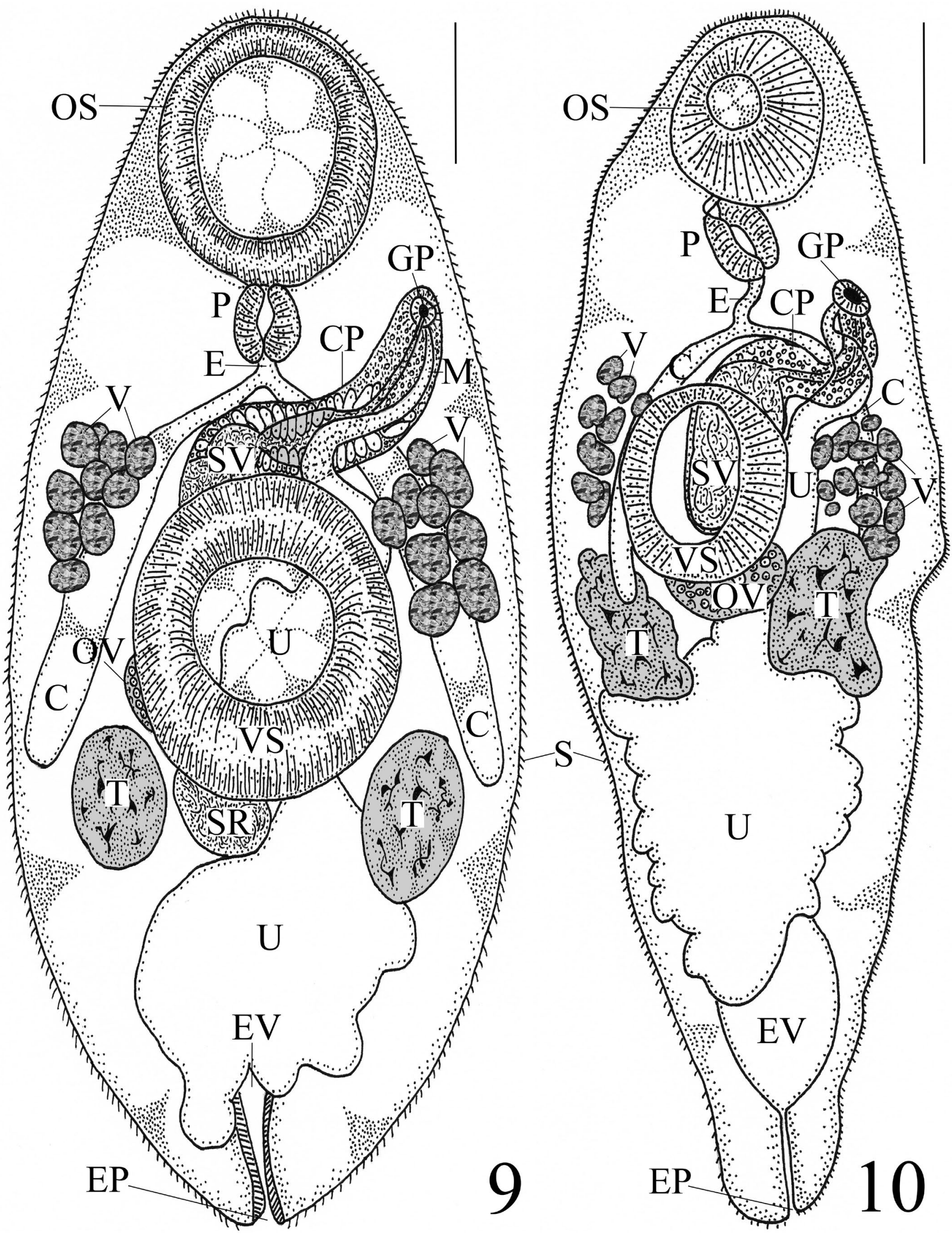


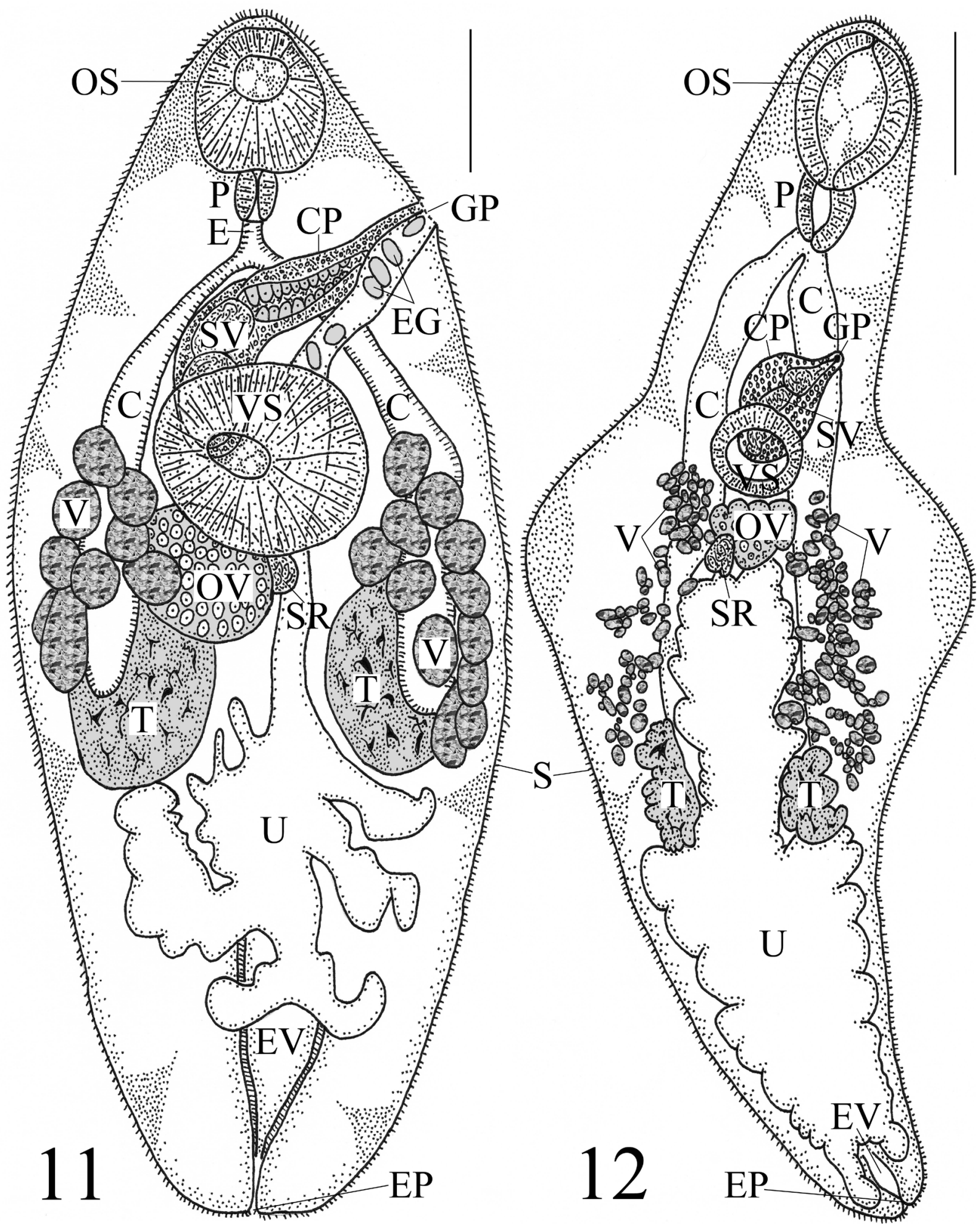

Figures 11-12. Steganoderma szidati Viozzi, Flores and Ostrowski de Núñez, 2000 and Steganoderma atherinae (Price, 1934) Manter, 1947 (Digenea: Zoogonidae: Lepidophyllinae). 11. S. szidati, ventral view, redrawn from Viozzi et al. (2000, fig. 1a). 12. S. atherinae, ventral view, redrawn from Price (1934, fig. 3). Abbreviations: C, Cecum; CP, Cirrus pouch; E, Esophagus; EG, Egg; EP, Excretory pore; EV, Excretory vesicle; GP, Genital pore; OS, Oral sucker; OV, Ovary; P, Pharynx; S, Spines; SR, Seminal receptacle; SV, Seminal vesicle; T, Testis; U, Uterus; V, Vitellarium; VS, Ventral sucker. Scale bars: Fig. $11=175 \mu \mathrm{m}$; Fig. $12=150 \mu \mathrm{m}$. 


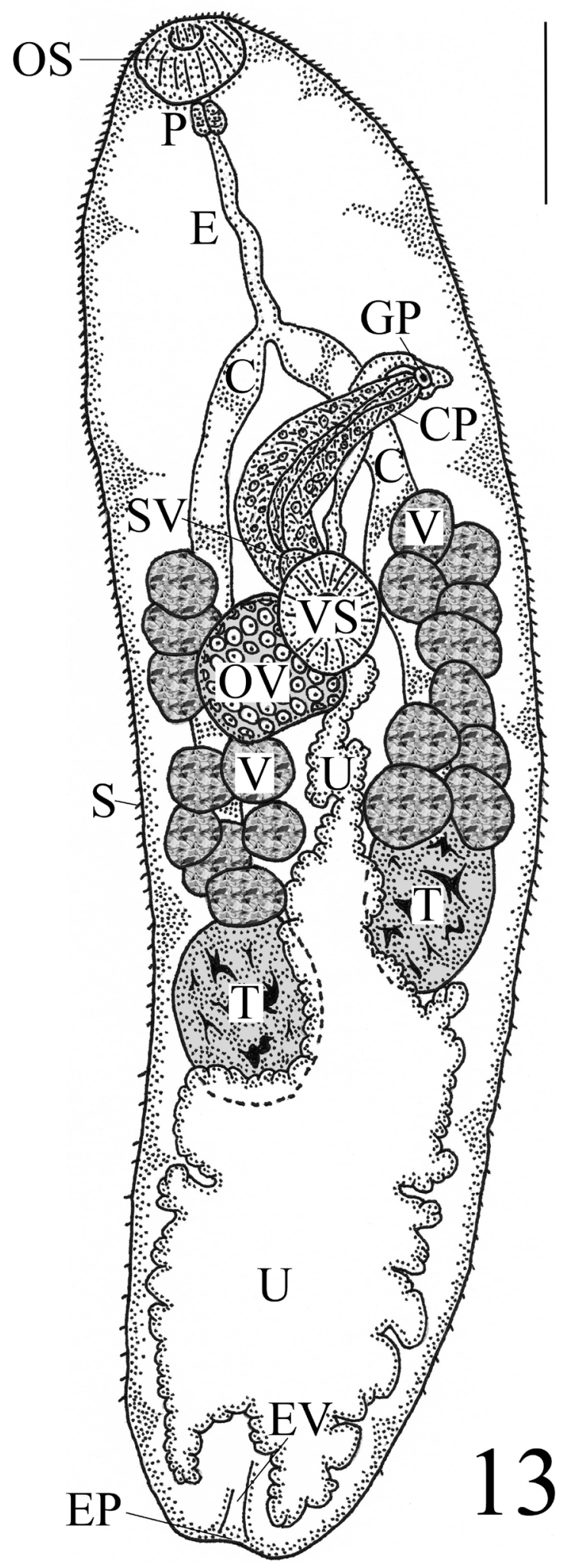

Figure 13. Steganoderma formosum Stafford, 1904 (Digenea: Zoogonidae: Lepidophyllinae), ventral view, redrawn from Bray (1987, fig. 12A; 2008b, fig. 64.21). Abbreviations: C, Cecum; CP, Cirrus pouch; E, Esophagus; EP, Excretory pore; EV, Excretory vesicle; GP, Genital pore; OS, Oral sucker; OV, Ovary; P, Pharynx; S, Spines; SV, Seminal vesicle; T, Testis; U, Uterus; V, Vitellarium; VS, Ventral sucker. Scale bar: $325 \mu \mathrm{m}$. 
rubrivinctus represents a new host record in that this is the first report of a digenean from this host species (see Table 1). There is some question about the proper identification of this host. Froese and Pauly (2019) state that Se. rubrivinctus is distributed in the Eastern Pacific from San Francisco, California, south to Cape San Quintin, Baja California, Mexico. The host for this study was collected a relatively short distance north off Newport, Oregon. While it is possible that this fish may have been misidentified (we have no way to confirm the identity of this fish, as the host was not saved), it is also feasible that this particular fish may have moved farther north within the chilly deeper waters off the west coast of the US where it was captured (it inhabits depths to $302 \mathrm{~m}$ [see Froese and Pauly, 2019] and our specimen was from $200 \mathrm{~m}$ depth). In addition, the slide containing the digenean specimen (HWML \#42863) has handwritten on it "Sebastodes rubrivnt." (Sebastodes rubrivinctus [Jordan and Gilbert, 1880] is a synonym of Sebastes rubrivinctus).

In our earlier comparison of S. eamiqtrema to the seven other recognized species of Steganoderma, we noted variability in the features, measurements, morphometric percentages, and ratios reported in previous redescriptions and/or supplemental descriptions of the type species, $S$. formosum, from the Atlantic Ocean (see Table 3). From the limited type description of $S$. formosum (see Stafford, 1904), the type material measured $2,600 \times 810$ in size; however, in later-described material, the body size of S. formosum varied: 3,250 $\times 860$ (Manter, 1926, figs. 5860); 1,750-2,350 × 620-1,000 (Linton, 1940, figs. 278-282); 2,690 × 760 (Miller, 1941, fig. 20); 4,105 × 1,224 (Polyanksii, 1955, fig. 22); 2,500-3,050 × 650-750 (Ronald, 1960); and 2,772 × 707 (Bray, 1979, 1987, fig. 12A). Manter (1926, p. 89) described within the parenchyma "numerous, conspicuous, round to oval bodies" (13-39 $\mu \mathrm{m}$ in diameter) in the anterior region, particularly the vicinity of the esophagus, of $S$. formosum and these cells exhibited "a very strong affinity for eosin stain." Linton (1940, p. 48 and fig. 280) also described and illustrated these cells from the "neck" (i.e., forebody) of this species; his material possessing "round, oval, and pyriform granular bodies" measuring 10-30 $\mu \mathrm{m}$ in diameter which he described as "characteristic of this species" (see also fig. 22 of Polyanskii, 1955). We found no evidence of these cells in the other redescriptions of $S$. formosum examined. With the exception of $S$. formosum redescribed by Miller (1941) (sucker width ratio 1:1.33) and Bray (1987, fig. 12A) (sucker length ratio 1:1.43), both suckers were reported to be about equal in size in this species. Manter (1926, p. 89) stated that in S. formosum the posterior end of the esophagus splits into two short branches each about $46 \mu \mathrm{m}$ long, thus "the intestine proper" does not begin at the bifurcation but histologically extends slightly beyond this point; we saw no evidence of these "two short branches" in other redescriptions of S. formosum. Ceca length of $S$. formosum varied across the reports we examined: in the type description of Stafford (1904, p. 486) they "end at half the length or slightly more of the body"; Manter (1926, p. 88) stated that the ceca extended "slightly more than half the body length," and his fig. 58 shows them terminating a short distance anterior to the testes; the ceca could be traced back only to the level of the posterior edge of the ventral sucker in S. formosum of Linton (1940, fig. 278) [Note: the left cecum does extend to the left testis in fig. 280 of Linton, 1940]; and in Miller (1941) the ceca extend to the anterior margins of the testes as they appear to do in fig. 22 of Polyanskii (1955) and in fig. 12A of Bray (1987). We also note that the ceca illustrated in the material of Bray (1987, fig. 12A) appears noticeably more thick-walled and the testes are illustrated more diagonal than opposite when compared to previously redescribed specimens of S. formosum. Manter (1926) described the cirrus pouch of $S$. formosum as extending posteriorly to and sometimes overlapping the ventral sucker (this configuration also seen in Linton, 1940; Miller, 1941; Bray, 1987), while Polyanskii (1955, fig. 22) clearly illustrated an entirely pre-acetabular cirrus pouch. Manter (1926, p. 90) described the seminal vesicle of S. formosum as "poorly developed," Polyanskii $(1955$, fig. 22) ambiguously portrayed it as a single? elongated object in the proximal portion of the cirrus pouch, and Bray (1987, fig. $12 A)$ illustrated it as a single saccate feature. Ching (1960, p. 242, fig. 1) observed a well-developed, bipartite seminal vesicle "divided into a small and large portion" in two specimens of S. formosum collected from the English sole, Parophrys vetulus Girard, 1854 (Pleuronectiformes: Pleuronectidae), from Friday Harbor, WA-a location relatively near where our specimens of $S$. eamiqtrema were found off Oregon. As we will elaborate, we are doubtful of the identification of this material as $S$. formosum because of its location being from the Pacific and not the Atlantic Ocean (i.e., Ching, 1960 stated that her report was the first time S. formosum had been reported along the Pacific coast of North America). This material also possesses "vitellaria [that] are more diffuse, not distinctly divided into follicles" (Ching, 1960, p. 242). Unfortunately, Ching deposited in the USNM only type material of new species from her paper, and online records of $S$. formosum in the USNM show holdings only from off the Atlantic coast of USA. Linton (1940, p. 48) described the genital pore as "on a level with the forking of the intestine" (i.e., bifurcal; cf. Miller, 1941), but figs. 278 and 280 clearly illustrate it as post-bifurcal, as does fig. 22 of Polyanskii (1955) and possibly fig. 12A of Bray (1987). 
The ovary in this species was originally described by Stafford (1904) as to the right of and overlapping the ventral sucker. Manter (1926, fig. 58) described the ovary as completely posterior to the ventral sucker and either median or to one side, while it is at the right lateral border of the ventral sucker in Linton (1940) and Miller (1941). The ovary was illustrated by Polyanskii (1955, fig. 22) further anterior relative to the ventral sucker (i.e., the anterior half of it pre-acetabular), and Bray (1987, fig. 12A) illustrated the ovary in a relatively intermediate position-the anterior half overlapped by the posterior half of the ventral sucker. In addition, while the majority of the reports described and illustrated the ovary with an ovate or globular shape, this feature was described by Linton (1940, p. 48) as "somewhat subtriangular," and it appears bean-shaped in his fig. 280 . Polyanskii (1955, fig. 22) illustrated a very large, almost rectangular-shaped, seminal receptacle $(299 \times 582)$ apparently filled with sperm in $S$. formosum collected from the Barents Sea, yet no information was given on this feature by other authors (see Table 3 and Bray, 1987, p. 108-110, who offers a brief discussion on the difficulty in observing the seminal receptacle in this species). We noted variability in the number of vitelline follicles in S. formosum, including as many as $8-9,9-13$, and $10-12$ follicles per worm in the dextral/aporal field and 6-16, 9, 10, and 10-12 follicles per worm, respectively, in the sinistral/poral field (Stafford, 1904; Manter, 1926; Linton, 1940, figs. 278, 280; Miller, 1941, fig. 20; Polyanskii, 1955, fig. 22; Bray, 1987, fig. 12A; see our Table 3). Egg size was consistent in redescriptions of S. formosum (see Table 3): $34 \times 17$ (Manter, 1926); 30-39 $\times$ 15-24 (Linton, 1940); $36 \times 17$ (Miller, 1941); and 30-39 × 12-20 (Ronald, 1960). The excretory vesicle was seldom described and/or illustrated in prior redescriptions of $S$. formosum because we suspect, in part, occlusion by eggs in the uterus. However, Manter (1926, p. 89, figs. 59, 60) provided a detailed description of this feature in his specimens where he described the vesicle extending from the posterior extremity almost to the posterior margin of the ventral sucker "where it spreads out laterally, T-like"; near its anterior end it becomes swollen enough to fill the larger part of a cross-section of the body in that region and comes in close contact with the ceca from which it is separated by a very narrow distance. Now, as we take a breath and consider the abundance of variation just described, we must contemplate, at this point, if these observations either indicate intraspecific variation or could they be suggestive of a lack of conspecificity among "S. formosum" within the Atlantic (i.e., cryptic species)? Furthermore, what if we consider these same questions as they relate to reports of this species from other ocean basins (i.e., helminth conspecificity in the Pacific vs. Atlantic Oceans)?
We speculated on this question further, particularly as it related to a hypothesis proposed by Bray (1987, p. 115) that $S$. formosum is "probably circum-arctic-boreal." Bray (1987) listed S. formosum as distributed in the North Pacific (Petropavolvsk region, Sea of Okhotsk, Sea of Chukotsk, Kuril Islands, Sea of Japan, Kamchatka, Bering Sea, British Columbia, Washington State), Barents Sea, and NW Atlantic Ocean (east coasts of Canada, Maine, and Massachusetts); we note as did Bray (1987) that several of these reports were of progenetic metacercaria encysted in the musculature of decapod crustaceans and not of adults infecting fish. Bray (1987, p. 108) also listed several families of fish whose members have been reported as definitive hosts for S. formosum, including the Agonidae (poachers), Bothidae (lefteye flounders), Cottidae (sculpins), Cyclopteridae (lumpfishes), Gadidae (cods and haddocks), Pleuronectidae (righteye flounders), Rajidae (skates-accidental infection), Scorpaenidae (scorpionfishes or rockfishes), and the Squalidae (dogfish sharks-accidental infection). We are struck by this wide geographic and host variability for S. formosum - might these identifications be based on the idea that digeneans are so plastic in their morphology that even if characteristics differ (Table 3), some authors may consider all somewhat similar worms to be of the same species? Were these decisions based on comparisons made without all available morphologic, ecological, and molecular information and, if so, is this "classic" (i.e., old-fashioned, outdated?) viewpoint of species still efficacious today? If we want to have the full extent of biodiversity identified, we cannot ignore morphologic, ecological, or molecular differences that demonstrate that specimens from different species of hosts and from geographically separate localities may represent different lineages that have been separated for a long geological period. Indeed, it is inconceivable to us that there could be gene flow between helminths in the North Atlantic (i.e., $S$. formosum sensu stricto, see Table 3) and those in the North Pacific, and with the host species in each ocean different as well (Bray, 1987). Studies have used rigorous techniques to identify various species within a "species group" that had, at one time, been considered all one variable species, and even past publications of different descriptions appear to support the hypothesis that these helminths (i.e., S. formosum sensu (ato) may be part of a species complex and not the same worldwide species. Finally, we note that Bray (1987) listed two definitive hosts for S. formosum from Sebastes (Sebastidae, formerly Scorpaenidae), the genus of host examined in the present study: the rougheye rockfish, Sebastes aleutianus (Jordan and Evermann, 1898), and the Pacific ocean perch, Sebastes alutus (Gilbert, 1890). Both Se. aleutianus and Se. alutus are found in the North Pacific 
from Japan to the Bering Sea and throughout the Aleutian Islands south to San Diego, California, from depths down to $825 \mathrm{~m}$, respectively (Froese and Pauly, 2019). As both species co-occur with Se. elongatus in the deeper waters off Oregon, we suspect that what was reported as $S$. formosum from Se. aleutianus and Se. alutus may be $S$. eamiqtrema.

Steganoderma is found in the deep sea. With this report, we are aware of at least two accepted species of Steganoderma from deeper waters, S. formosum and $S$. eamigtrema, as well as the unidentified Steganoderma sp. described herein (see also Blend et al., 2020). Klimpel et al. $(2001,2009)$ documented that S. formosum has a bathymetric range of $183-732 \mathrm{~m}$ and it is known from three hosts in the deep sea. Sekerak and Arai (1977) reported this species from the rougheye rockfish, Se. aleutianus, in the NE Pacific Ocean [Note: based on our earlier comments, this helminth identification may not be accurate], while Scott (1987) recorded it from the red hake, Urophycis chuss (Walbaum, 1792), and the white hake, Urophycis tenuis (Mitchill, 1814) (Gadiformes: Phycidae), found along the Scotian Shelf. Thus, the finding of S. eamiqtrema and Steganoderma sp. in this study comes as no surprise as rockfish (i.e., Sebastidae) are not a new host group for Steganoderma in the deep; this parasite genus having been documented from Se. aleutianus, Se. alutus, Se. elongatus, and Se. rubrivinctus, which can inhabit depths from 25-900 m, 0-825 m, 25-425 m, and 0-302 m, respectively (Sekerak and Arai, 1977; Bray, 1987; Klimpel et al., 2001, 2009; Froese and Pauly, 2019; this study). As stated earlier, Bray (1987) listed a plethora of definitive hosts for $S$. formosum, including various species of pleuronectids, cottids, gadids, sebastids, agonids, paralichthyids, and liparids as well as accidental infections in rajids and squalids. Upon closer examination of these definitive hosts (see Froese and Pauly, 2019), we found many that inhabit depths below $200 \mathrm{~m}$ (i.e., considered the deep sea), suggestive that deeper individuals of these host species should be examined for species of Steganoderma (e.g., the Kamchatka flounder, Atheresthes evermanni Jordan and Starks, 1904 [Pleuronectiformes: Pleuronectidae]; the armorhead sculpin, Gymnocanthus galeatus Bean, 1881 [Scorpaeniformes: Cottidae]; the Pacific cod, Gadus macrocephalus Tilesius, 1810, and the Atlantic cod, Gadus morhua Linnaeus, 1758 [Gadiformes: Gadidae]; Se. aleutianus and Se. alutus [Scorpaeniformes: Sebastidae]; the sturgeon poacher, Podothecus accipenserinus [Tilesius, 1813] [Scorpaeniformes: Agonidae]; the American fourspot flounder, Hippoglossina oblonga [Mitchill, 1815] [Syn. Paralichthys oblongus (Mitchill, 1815)] [Pleuronectiformes: Paralichthyidae]; and a snailfish species, Careproctus sp.).
As we are aware of only two nominal species of Steganoderma inhabiting the deep sea (S. eamiqtrema and $S$. formosum), conclusions regarding host specificity, especially in deeper waters, are limited. Bray (1987) listed four categories of host specificity for zoogonids: Category $1-$ strict oioxenic specificity; Category 2-strict stenoxenic specificity; Category 3-predominant stenoxenic specificity; and Category 4-euryxenic specificity. He classified S. formosum as Category 3 (see Table III of Bray, 1987), and this includes species that exhibit a strong predilection for fish in one taxonomic group but are occasionally recorded in species from other groups. Indeed, Bray (1987, Table III) noted pleuronectids as the predominant host group for S. formosum, yet gadids and scorpaeniformes also are listed as other hosts. Accidental infections can occur when a parasite survives in a piscivorous fish that ingests either a normal definitive host or possibly an intermediate host containing a progenetic metacercaria; S. formosum is a good example, having accidentally infected the barndoor skate, Dipturus laevis (Mitchill, 1818) (Syn. Raja laevis Mitchill, 1818) (Rajiformes: Rajidae), and the piked dogfish, Squalus acanthias Linnaeus, 1758 (Squaliformes: Squalidae) (Bray, 1987). Looking across the eight species that we recognize in Steganoderma, we see an "apparent" range of host specificity, but this could be misleading. Category 3-predominant stenoxenic specificity-is exhibited by $S$. formosum, and at first glance, Steganoderma spp. within two other zoogonid host specificity categories appear apparent. Category 1 -strict oioxenic specificity-defined as those zoogonid species recorded from one fish species only; in this case, S. valchetensis, known only from the naked characin, Gymnocharacinus bergi (Characidae), from northern Patagonia, Argentina (Etchegoin et al., 2002), and S. eamiqtrema, known only from the greenstriped rockfish, Se. elongatus (Sebastidae), from off Oregon (this study). Also, Category 2-strict stenoxenic specificity-defined as those zoogonid species restricted to a single supra-specific fish group (i.e., genus, family, and order); S. szidati is known to infect only the inanga, Galaxias maculatus, and the galaxiid Galaxias platei (Osmeriformes: Galaxiidae) from Lake Gutiérrez and other glacial lakes of Andean Patagonia, Argentina (Viozzi et al., 2000). However, Bray (1987) pointed out a critical caveat in the use of these categories; only those zoogonid species recorded more than once can be studied in relation to specificity, and the number of times a species has been recorded will be directly related to the reliability of the categorization. We are aware only of the published type descriptions of S. valchetensis and S. szidati (Viozzi et al., 2000; Etchegoin et al., 2002) and have found no subsequent reports of either zoogonid species infecting the same and/or different 
hosts. However, prior reports of S. eamiqtrema may exist based on speculations we presented earlier (see Sekerak and Arai, 1977; Bray, 1987 as relates to his listing of "S. formosum" from Se. aleutianus and Se. alutus) allowing higher reliability in categorization. More research is needed, particularly with host species of "apparent" Category 1 and 2 members of Steganoderma.

Only one species of Steganoderma, Steganoderma gibsoni Cribb, Bray and Barker, 1992, has any gene sequence information in GenBank. Currently available are partial sequences of the 5.8S rDNA and 28S rDNA genes as well as a complete sequence of the ITS-2 gene from S. gibsoni (see Shimazu et al., 2014). However, this species is no longer in Steganoderma, and it is considered now a member of Lecithostaphylus (see WoRMS, 2020a); thus, there are no DNA gene sequences currently on GenBank from accepted species of Steganoderma. This finding is surprising to us given the wide range of definitive hosts and geographic distribution for "S. formosum." Clearly, more molecular work is needed with this genus (e.g., sampling and sequencing of S. formosum sensu lato from various localities and hosts to test for the presence of a species complex, etc.).

Our literature searches as well as this study allow us to speculate on life history strategies for species of Steganoderma in the deep sea. The two host species in our study, Se. elongatus and Se. rubrivinctus, overlap in their distributions; both found in the NE Pacific Ocean along the west coast of North America from San Francisco, California, south to Cape San Quintin, Baja California, Mexico (Froese and Pauly, 2019). A demersal species, Se. elongatus inhabits both inshore and offshore areas and can be found on rocky and soft bottoms down to $425 \mathrm{~m}$; whereas, Se. rubrivinctus, also a demersal species, inhabits only rocky areas down to $302 \mathrm{~m}$ depth. Froese and Pauly (2019) provide for neither Se. elongatus nor Se. rubrivinctus any information on diet/food items; however, two other rockfish species, Se. aleutianus and Se. alutus, have similar distributions compared to Se. elongatus and Se. rubrivinctus (NE Pacific Ocean), are known to harbor Steganoderma, and have diet/food item information available (see Sekerak and Arai, 1977; Bray, 1987; Klimpel et al., 2001, 2009; Froese and Pauly, 2019). The rougheye rockfish, Se. aleutianus, preys on marine arthropods such as crabs (Chionoecetes bairdi Rathbun, 1924); identified (Themisto sp.) and unidentified amphipods; identified (Thysanoessa inermis [Krøyer, 1846], Thysanoessa raschii [M. Sars, 1864]) and unidentified euphausiids; and unidentified isopods, mysids, shrimps, and prawns as well as on other taxa of marine organisms including unidentified and identified bony fish (e.g., the eulachon smelt, Thaleichthys pacificus [Richardson, 1836]) and unidentified squids, cuttlefish, bivalves, polychaetes, and other planktonic invertebrates (Froese and Pauly, 2019). The Pacific ocean perch, Se. alutus, preys on unidentified and identified bony fish (e.g., deepsea smelts such as the northern smoothtongue, Leuroglossus schmidti Rass, 1955; the bigeye lanternfish, Protomyctophum thompsoni [Chapman, 1944]; Alaska pollock, Gadus chalcogrammus Pallas, 1814) as well as a veritable smorgasbord of unidentified shrimps (adult and larvae; unspecified Pandalidae Haworth, 1825), prawns, squids, cuttlefish, amphipods, benthic and planktonic (i.e., calanoid) copepods, gastropods, euphausiids, mysids, ctenophores, and other unidentified planktonic invertebrates (Froese and Pauly, 2019). We noted that Polyanskii (1955, p. 57), who examined the parasites of fish from the Barents Sea, stated that "Uspenskaya (1952) reported that halibut [i.e., presumed to be the Atlantic halibut, Hippoglossus hippoglossus, which is the type host for S. formosum - see Stafford, 1904] infestations [of S. formosum] followed consumption of bottom-living decapods of [the] genus Sclerocrangon [G. O. Sars, 1883], the second intermediate host of $S$. messjatzevi [= S. formosum]." Bray (1987) listed the following second intermediate hosts, all crustacean decapods, for S. formosum: caridean shrimps (Argis lar [Owen, 1839]; Pandalus borealis Krøyer, 1939; Pandalus dispar [Rathbun, 1902] [Syn. Pandalopsis dispar Rathbun, 1902]; Pandalus goniurus Stimpson, 1860; Sabinea septemcarinata [Sabine, 1824]; Sclerocrangon boreas [Phipps, 1774]; Sclerocrangon salebrosa [Owen, 1839]), hermit crabs (Pagurus pubescens Krøyer, 1838), and possibly oregoniid crabs (Chionoecetes opilio [O. Fabricius, 1788]). Marcogliese (1996) reported progenetic metacercariae of $S$. formosum in the Acadian hermit crab, Pagurus acadianus Benedict, 1901, from the Scotian Shelf east of Nova Scotia, Canada. Bray et al. (1999) stated that marine zoogonids utilize gastropods (e.g., nassariids) as a first intermediate host while brittle stars, polychaetes, bivalves, and gastropods are second or subsequent intermediate hosts. Based on the known diet of the two co-occurring sebastid rockfish species, Se. aleutianus and Se. alutus, and the overlap in these prey items with known host groups for marine zoogonids (see Bray, 1987; Table 6 of Bray et al., 1999), we can speculate that the life cycle of S. eamiqtrema and Steganoderma sp. in the definitive hosts Se. elongatus and Se. rubrivinctus within the deep sea, respectively, may involve a gastropod as a first intermediate host and possibly caridean shrimps (e.g., Pandalidae, Sclerocrangon spp.), oregoniid crabs (e.g., Chionoecetes spp.), polychaetes, bivalves, and/or gastropods as second or subsequent intermediate hosts.

Steganoderma contains eight species (WoRMS, 2020b; Blend et al., 2020; Present study); four of these are found in marine waters and the remaining four species inhabit 
freshwater. While the four marine species (S. atherinae, $S$. eamiqtrema, $S$. formosum, S. rhiphidium) were originally described from the NW Atlantic Ocean off Canada and the Dominican Republic as well as from the North Pacific Ocean off China and Oregon, respectively, the four freshwater species (S. macrophallus, S. oviformis, S. szidati, S. valchetensis) were all originally described from Argentina. Given the differences in intermediate hosts available to piscine definitive hosts in freshwater environments (Note: Szidat, 1962 stated that insects and their larvae were almost exclusively the only prey items found in the stomach of fish [i.e., Aplochiton zebra (Galaxiidae Bonaparte)] parasitized by $S$. oviformis, however, A. zebra is amphidromous; Viozzi et al., 2000 described S. szidati from the galaxiids, Galaxias maculatus [catadromous] and G. platei [amphidromous]) vs. marine-especially deep-sea-environments, we suspect that with further molecular, morphological, and life history work, the genus Steganoderma will be taxonomically divided up.

Acknowledgments - We gratefully acknowledge the foresight of the late Dr. James E. McCauley, Dept. of Oceanography, Oregon State Univ., Corvallis, Oregon, for collecting and depositing this material. We thank Dr. Scott L. Gardner, curator and professor, HWML, for his editorial assistance in supporting this work and Dr. Norman O. Dronen, Dept. of Ecology and Conservation Biology, as well as the Interlibrary Loan Division of the Evans Library, Texas A\&M Univ., College Station, Texas, for helping CKB obtain needed literature. We are indebted to Mr. Yasser F. M. Karar, Dept. of Zoology, New Valley Univ., Kharga, New Valley, Egypt, for his help in deriving the Arabic name for the new species described herein. Our continued thanks go to Gordon College, Wenham, Massachusetts, for the extended loan of microscopes and other equipment to CKB. We also acknowledge the Corpus Christi Museum of Science and History for their ongoing support of this research, and we thank the two anonymous reviewers whose constructive comments and suggestions improved this manuscript. This study was completed and funded as part of a sabbatical approved for CKB by the Faculty Development Committee, Gordon College.

\section{References}

Acena, S. P. 1941. Preliminary notes on a trematode with two yolk reservoirs and a new species of Lecithochirium. Philippine Journal of Science 75: 285-289.

Alvarado-Villamar, M. R., and Ruiz-Campos, G. 1992. Comparative study of the incidence of macroparasites in six species of Sebastes (Pisces, Scorpaenidae) from the northwestern coast of Baja California, Mexico. Ciencias Marinas 18: 79-92.

Annereaux, R. F. 1947. Three new trematodes from marine fishes of California. Transactions of the American Microscopical Society 66: 249-255.

Arai, H. P. 1969. Preliminary report on the parasites of certain marine fishes of British Columbia. Journal of the Fisheries Research Board of Canada 26: 2319-2337.

Blend, C. K., Racz, G. R., and Gardner, S. L. 2020. Gaharitrema droneni n. gen., n. sp. (Digenea: Zoogonidae) from the pudgy cuskeel, Spectrunculus grandis (Ophidiiformes: Ophidiidae), from deep waters off Oregon, with updated keys to zoogonid subfamilies and genera. Journal of Parasitology 106: 235-246.

Bray, R. A. 1979. Digenea in marine fishes from the eastern seaboard of Canada. Journal of Natural History 13: 399-431.

Bray, R. A. 1987. A revision of the family Zoogonidae Odhner, 1902 (Platyhelminthes: Digenea): Subfamily Lepidophyllinae and comments on some aspects of biology. Systematic Parasitology 9: 83-123.

Bray, R. A. 2008a. Superfamily Microphalloidea Ward, 1901. Pages 447-450. In: Keys to the Trematoda, Volume 3. Ed. by Bray, R. A., Gibson, D. I., and Jones, A. CAB International and Natural History Museum. 824 pp.

Bray, R. A. 2008b. Family Zoogonidae Odhner, 1902. Pages 605-629. In: Keys to the Trematoda, Volume 3. Ed. by Bray, R. A., Gibson, D. I., and Jones, A. CAB International and Natural History Museum. 824 pp.

Bray, R. A., and Gibson, D. I. 1990. The Lepocreadiidae (Digenea) of fishes of the north-east Atlantic: review of the genera Opechona Looss, 1907 and Prodistomum Linton, 1910. Systematic Parasitology 15: 159-202.

Bray, R. A.,, Littlewood, D. T. J., Herniou, E. A., Williams, B., and Henderson, R. E. 1999. Digenean parasites of deepsea teleosts: a review and case studies of intrageneric phylogenies. Parasitology 119(Suppl.): S125-S144.

Bush A. O., Lafferty, K. D., Lotz, J. M., and Shostak, A. W. 1997. Parasitology meets ecology on its own terms: Margolis et al. revisited. Journal of Parasitology 83: 575-583.

Chapa, E. 1969. Studies on digenetic trematodes of marine fishes from Monterey and Tamales Bays, California. MS Thesis. University of the Pacific, Stockton, California. $80 \mathrm{pp}$.

Chapa, E. 1976. Personal Communication. Dept. of Biology, University of the Pacific, Stockton, California. [see Love and Moser, 1976, 1983] 
Ching, H. L. 1960. Some digenetic trematodes of fishes of Friday Harbor, Washington. Journal of Parasitology 46: 241-250.

Crane, J. W. 1972. Systematics and new species of marine Monogenea from California. The Wassmann Journal of Biology 30: 109-166.

Cutmore, S. C., Miller, T. L., Bray, R. A., and Cribb, T. H. 2014. A new species of Plectognathotrema Layman, 1930 (Trematoda: Zoogonidae) from an Australian monacanthid, with a molecular assessment of the phylogenetic position of the genus. Systematic Parasitology 89: 237-246.

Dailey, M. D., Jensen, L. A., and Hill, B. W. 1980. Larval anisakine roundworms of marine fishes from southern and central California, with comments on public health implications. California Fish and Game 67: 240-245.

Eagle, R. J., and McCauley, J. E. 1964. Collection of helminths from marine abyssal fishes. Journal of Parasitology 50: 37. [Abstract]

Eagle, R. J., and McCauley, J. E. 1965. Collecting and preparing deep-sea trematodes. Turtox News 43: 220-221.

Egorova, T. P. 1994a. Composition of the genus Megalocotyloides (Capsalidae) and description of $M$. sebastodi sp. nov. of scorpaenid fishes from the Pacific. Izvestiya of the Pacific Research Institute of Fisheries and Oceanography (TIN-RO), Vladivostok 117: 148-153. [In Russian]

Egorova, T. P. 1994b. A taxonomic review of the subfamily Trochopodinae (Monogenoidea: Capsalidae). Parazitologiya, Akademiya Nauk SSSR, Leningrad 28: 81-91. [In Russian]

Etchegoin, J. A., Cremonte, F., and Escalante, A. H. 2002. Steganoderma (Steganoderma) valchetensis n. sp. (Digenea: Zoogonidae) from the relict fish Gymnocharacinus bergi (Osteichthyes: Characidae) in Argentina. Systematic Parasitology 51: 149-153.

Fernandez, V., Semenas, L., and Viozzi, G. 2012. Parasites of the "Peladilla," Aplochiton zebra (Osmeriformes: Galaxiidae), from Patagonia (Argentina and Chile). Comparative Parasitology 79: 231-237.

Froese, R., and Pauly, D. 2019. FishBase (version 12/2019). Available at: www.fishbase.org. Accessed 8 June 2020.

Gibson, D. I. 1996. Guide to the parasites of fishes of Canada. Part IV. Trematoda. Ed. by Margolis, L., and Kabata, Z. Canadian Special Publication of Fisheries and Aquatic Sciences, No. 124, NCR Research Press, Ottawa, Canada. 373 pp.

Jensen, L. A. 1975. Personal communication. 17639 Clymer St., Granda Hills, California, 91344. [see Love and Moser, 1976, p. 307, 476]

Jensen, L. A. 1977. Personal communication. Dept. of Biological Sciences, University of Northern Colorado, Greeley, Colorado. [see Love and Moser, 1983, p. 355, 571]
Kabata, Z. 1970. Some Lernaeopodidae (Copepoda) from fishes of British Columbia. Journal of the Fisheries Research Board Canada 27: 865-885.

Kabata, Z. 1973. The species of Lepeophtheirus (Copepoda: Caligidae) from fishes of British Columbia. Journal of the Fisheries Research Board of Canada 30: 729-759.

Kazachenko, V. N. 1986. Parasitic copepods (Crustacea, Copepoda) on fish of the genus Sebastes (Scorpaenidae). Trudy Zoologicheskogo Instituta Akademiya Nauk SSSR 155: 155-169. [In Russian]

Kazachenko, V. N., Korotaeva, V. D., and Kurochkin. J. V. 1972. Parasitic Crustacea of some fishes of the Pacific Ocean. Izvestiya Tikhookeanskogo Nauchno-issledova-tel'skogo Instituta Rybnogo Khozyaistvai Okeanografii (TIN-RO) 81: 224-238. [In Russian]

Kent, M. L., Traxler, G. S., Kieser, D., Richard, J., Dawe, S. C., Shaw, R. W., Prosperi-Porta, G., Ketcheson, J., and Evelyn, T. P. T. 1998. Survey of salmonid pathogens in oceancaught fishes in British Columbia, Canada. Journal of Aquatic Animal Health 10: 211-219.

Klimpel, S., Busch, M. W., Kellermanns, E., Kleinertz, S., and Palm, H.-W. 2009. Metazoan deep-sea fish parasites. Verlag Natur and Wissenschaft, Solingen, Germany. 384 pp.

Klimpel, S., Seehagen, A., Palm, H.-W., and Rosenthal, H. 2001. Deepwater metazoan fish parasites of the world. Logos Verlag, Berlin, Germany. 315 pp.

Linton, E. 1940. Trematodes from fishes mainly from the Woods Hole region, Massachusetts. Proceedings of the United States National Museum 88: 1-172.

Love, M. S., and Moser, M. 1976. Parasites of California marine and estuarine fish. University of California-Santa Barbara, Santa Barbara, California, USA. 517 pp.

Love, M. S., and Moser, M. 1983. A checklist of parasites of California, Oregon, and Washington marine and estuarine fishes. US Department of Commerce, NOAA Technical Report, NMFS No. SSRF-777. 576 pp.

Love, M. S., Yoklavich, M., and Thornsteinson, L. 2002. The rockfishes of the northeast Pacific. University of California Press, Berkeley, California, USA. 414 pp.

Manter, H. W. 1926. Some North American fish trematodes. Illinois Biological Monographs 10: 1-138.

Marcogliese, D. J. 1996. Evidence for specificity of Steganoderma formosum for its second intermediate host in the Northwest Atlantic. Journal of Helminthology 70: 215-218.

Margolis, L., and Arthur, J. R. 1979. Synopsis of the parasites of fishes of Canada. Bulletin of the Fisheries Research Board of Canada No. 199. 269 pp.

McCauley, J. E. 1964. A deep-sea digenetic trematode. Journal of Parasitology 50: 112-114.

McCauley, J. E. 1968. Six species of Lepidapedon Stafford, 1904 (Trematoda: Lepocreadiidae) from deep-sea fishes. Journal of Parasitology 54: 496-505. 
McCauley J. E., and Pequegnat, J. E. 1968. Two new species of Dinosoma Manter, 1934 (Trematoda: Hemiuridae) from deepwater macrourid fishes off the coast of Oregon. Journal of Parasitology 54: 931-934.

McFarlane, S. H. 1936. Study of the endoparasitic trematodes from marine fishes of Departure Bay, B.C. Journal of the Biological Board of Canada 2: 335-347.

Miller, M. J. 1941. A critical study of Stafford's report on "Trematodes of Canadian fishes" based on his trematode collection. Canadian Journal of Research 19: 28-52.

Moser, M., Love, M. S., and Jensen, L. A. 1976. Myxosporida (Protozoa) in California rockfish, Sebastes spp. Journal of Parasitology 62: 690-692.

Polyanskii, Yu. I. 1955. The parasitology of fish of northern marine waters on the USSR. Parasites of the fish of the Barents Sea. Trudy Zoologicheskogo Instituta Akademii Nauk SSSR 19: 5-170 [In Russian; English translation (1966), Israel Program for Scientific Translations, Jerusalem. 158 pp.]

Pratt, I., and McCauley, J. E. 1961. Trematodes of the Pacific northwest. An annotated catalog. Oregon State University Press, Corvallis, Oregon, USA. 118 pp.

Price, E. W. 1934. New digenetic trematodes from marine fishes. Smithsonian Miscellaneous Collections 91: 1-8.

Ronald, K. 1960. The metazoan parasites of the Heterosomata of the Gulf of St. Lawrence. VI. Digenea. Canadian Journal of Zoology 38: 923-937.

Scott, J. S. 1987. Helminth parasites of the alimentary tract of the hakes (Merluccius, Urophycis, Phycis: Teleostei) of the Scotian Shelf. Canadian Journal of Zoology 65: 304-311.

Sekerak, A. D. 1975. Parasites as indicators of populations and species of rockfishes (Sebastes: Scorpaenidae) of the Northwestern Pacific Ocean. PhD Thesis. University of Calgary, Calgary, Alberta. 251 pp.

Sekerak, A. D., and Arai, H. P. 1977. Some metazoan parasites of rockfishes of the genus Sebastes from the northeastern Pacific Ocean. Syesis 10: 139-144.

Shimazu, T., Cribb, T. H., Miller, T. L., Urabe, M., Van Ha, N., Thi Binh, T., and Shed'ko, M. B. 2014. Revision of Isoparorchis Southwell, 1913 (Digenea, Hemiuroidea, Isoparorchiidae), parasites of the air bladder of freshwater catfishes: a molecular and morphological study. Bulletin of the National Museum of Nature and Science, Series A (Zoology) 40: 15-51.

Stafford, J. 1904. Trematodes from Canadian fishes. Zoologischer Anzeiger 27: 481-495.
Stern, L., Myers, B. J., Amish, R. A., Knollenberg, W., and Chew, W. 1976. Anisakine nematodes in commercial marine fish from Washington State. Transactions of the American Microscopical Society 95: 264. [Abstract]

Szidat, L. 1962. Steganoderma oviformis n. sp. (Trematoda) del intestino de Haplochiton zebra Jenyns. Neotropica 8: 67-72.

Szidat, L., and Nani, A. 1951. Diplostomiasis cerebralis del Pejerrey. Una grave epizootia que afecta a la economía nacional producida por larvas de trematodes que desturyen el cerebro de los pejerreyes. Revista del Instituto Nacional de Investigacion de las Ciencias Naturales (Argentina) 1: 323-394.

Uspenskaya, A. V. 1952. Observations on the life cycle of Nordosstrema messjatzevi Issaitschikow. Doklady Akademii Nauk SSSR 85: 1419-1421. [In Russian]

Viozzi, G., Flores, V., and Ostrowski de Núñez, M. 2000. Steganoderma szidati n. sp. (Trematoda: Zoogonidae) from Galaxias maculatus (Jenyns) and G. platei Steindachner in Patagonia, Argentina. Systematic Parasitology 46: 203-208.

Wang, P.-Q. 1986. Some digenetic trematodes of fishes from Fujian [P]rovince, China. The Journal of Fujian Normal Teachers University (Natural Science) 2: 93-102.

Wilson, C. B. 1908. North American parasitic copepods: a list of those found upon the fishes of the Pacific coast, with descriptions of new genera and species. Proceedings of the United States National Museum 35: 431-481.

WoRMS. 2020a. Lecithostaphylus Odhner, 1911. In: World Register of Marine Species. Available at: http://www. marinespecies.org/aphia.php?p=taxdetails\&id $=108656$. Accessed 8 June 2020.

WoRMS. 2020b. Steganoderma Stafford, 1904. In: World Register of Marine Species. Available at: http://www. marinespecies.org/aphia.php?p=taxdetails\&id =108663\#. Accessed 8 June 2020.

Yamaguti, S. 1958. Systema helminthum. Vol 1. The digenetic trematodes of vertebrates. Part 1. Interscience Publishers, Inc., New York, New York, USA. 979 pp.

Yamaguti, S. 1963. Parasitic Copepoda and Branchiura of fishes. Interscience Publishers, Inc., New York, New York, USA. 1104 pp.

Yamaguti, S. 1971. Synopsis of digenetic trematodes of vertebrates. Vol 1. Keigaku Publishing Co., Tokyo, Japan. 1079 pp. 